University of Louisville

ThinkIR: The University of Louisville's Institutional Repository

$12-2010$

\title{
Social-cognitive predictors of physical activity initiation in type 2 diabetes following diabetes self-management education : application of the health action process approach.
}

\author{
Jason E. Bonner \\ University of Louisville
}

Follow this and additional works at: https://ir.library.louisville.edu/etd

\section{Recommended Citation}

Bonner, Jason E., "Social-cognitive predictors of physical activity initiation in type 2 diabetes following diabetes self-management education : application of the health action process approach." (2010). Electronic Theses and Dissertations. Paper 126.

https://doi.org/10.18297/etd/126

This Doctoral Dissertation is brought to you for free and open access by ThinkIR: The University of Louisville's Institutional Repository. It has been accepted for inclusion in Electronic Theses and Dissertations by an authorized administrator of ThinkIR: The University of Louisville's Institutional Repository. This title appears here courtesy of the author, who has retained all other copyrights. For more information, please contact thinkir@louisville.edu. 
SOCIAL-COGNITIVE PREDICTORS OF PHYSICAL ACTIVITY INITIATION IN TYPE 2 DIABETES FOLLOWING DIABETES SELF-MANAGEMENT EDUCATION: APPLICATION OF THE HEALTH ACTION PROCESS APPROACH

By

Jason E. Bonner

B.A., University of Southern Maine, 2002

M.A., University of Louisville, 2005

\begin{abstract}
A Dissertation
Submitted to the Faculty of the Graduate School of the University of Louisville in Partial Fulfillment of the Requirements for the Degree of
\end{abstract}

Doctor of Philosophy

Department of Psychological and Brain Sciences

University of Louisville

Louisville, Kentucky

December, 2010 
Copyright 2010 by Jason E. Bonner

All rights reserved 

SOCIAL-COGNITIVE PREDICTORS OF PHYSICAL ACTIVITY INITIATION IN TYPE 2 DIABETES FOLLOWING

DIABETES SELF-MANAGEMENT EDUCATION:

APPLICATION OF THE HEALTH ACTION PROCESS APPROACH

By

Jason E. Bonner

B.A., University of Southern Maine, 2002

M.A., University of Louisville, 2005

A Dissertation Approved on

September 1,2010

By the following Dissertation Committee

Barbara Stetson, Ph.D.

Dissertation Chair

Benjamın Mast, Ph.D.

Sri Prakash Mokshagundam, M.D.

Tamara Newton, Ph.D.

Paul Salmon, Ph.D. 


\section{DEDICATION}

This dissertation is dedicated to my wife and best friend, Krista M. Bonner. Thank you for your love, faith, and patience that nurtured this dream into existence. I remain eternally grateful for all that you have given of yourself through this journey. 


\section{ACKNOWLEDGEMENTS}

First, I would like to thank my dissertation chair and mentor Dr. Barbara Stetson for her patience, instruction, and guidance through my graduate training. I would also like to thank the members of my dissertation committee for their encouragement and guidance while navigating the dissertation process. I am grateful to Joseph Carhart for his invaluable support as the research assistant for this dissertation. A very special thanks is due to the diabetes educators and support personnel who made this study possible: Eloise Campbell, Andrea Grisanti, Michelle Smith, Michelle Hamilton, Debra Walker, Kim Jackson, Mary Kenealy, Beverly Breyette, Anna, Laura, and Kitty. I would also like to extend my gratitude for the assistance provided by Dr. Barbara Ainsworth, Dr. Ralf Schwarzer, and Dr. Pavel Zahorik. I wish to thank my brother Michael for his wit and wisdom as I walked down this long path. A tender thank you to my daughter Jillian, whose birth mid-way through this study yielded considerably less sleep, yet offered in return a sense of pride, contentment, and encouragement. I am grateful to my family and friends for their enduring unconditional love and support. Finally, I would like to thank my dog Rook, whose happy face and wagging tail since the early days of my graduate training has helped me traverse this journey without losing my sense of humor. 


\begin{abstract}
SOCIAL-COGNITIVE PREDICTORS OF PHYSICAL ACTIVITY INITIATION

IN TYPE 2 DIABETES FOLLOWING DIABETES SELF-MANAGEMENT

EDUCATION: APPLICATION OF THE HEALTH ACTION PROCESS APPROACH

Jason E. Bonner

September 1, 2010

Type 2 diabetes presents a public health crisis and a global pandemic. Successful management of diabetes requires engagement in a daily regimen of self-care behaviors to achieve optimal glycemic control and to reduce the severity of diabetes-related complications. Regular engagement in physical activity has been demonstrated to improve glycemic control and overall quality of life among patients with diabetes. Diabetes self-management education (DSME) has become the hallmark for instructing patients with diabetes to engage in physical activity, yet physical activity patterns of DSME patients remains largely unknown. Further, it is unclear what factors could account for such behavior change in the DSME setting.
\end{abstract}

Social-cognitive models of health behavior have attempted to explain behavior change such as physical activity initiation. One model, the Health Action Process Approach (HAPA), provides a parsimonious framework for understanding this process. The HAPA organizes key social-cognitive factors into a motivational stage, where a behavioral intention is formed, and a volitional stage, where self-regulatory processes such as action planning mediate the intention-behavior relationship, thereby translating 
intention into action. Using the HAPA as a theoretical framework, the present study sought to examine the key social-cognitive determinants of physical activity initiation among patients with type 2 diabetes after they participated in DSME.

A longitudinal, multi-site prospective study design utilized written and telephonebased surveys to assess HAPA constructs and physical activity in a DSME population. Participants were 152 adults with type 2 diabetes attending DSME classes in a mid-west metropolitan city. Results of this study revealed that several key social-cognitive factors, as conceptualized by the HAPA's motivational stage, predicted the formation of a behavioral intention to engage in physical activity. Findings on the HAPA volitional stage constructs indicated that only behavioral intention predicted which participants met the minimum amounts of physical activity promoted in DSME. Additionally, the present study revealed physical activity initiation remains a problem among DSME participants. Future research is recommended to clarify the causal role and pathways of socialcognitive factors in the HAPA model to better understand physical activity initiation within the DSME population. 
TABLE OF CONTENTS

\section{PAGE}

ACKNOWLEDGEMENTS

ABSTRACT

LIST OF TABLES

iv

$\mathrm{V}$

LIST OF FIGURES

xii

\section{INTRODUCTION}

Study Background, Purpose, Rationale

Type 2 Diabetes Mellitus 1

Physical Activity and Type 2 Diabetes 3

Social-Cognitive Models of Health Behavior $\quad 10$

Health Behavior Models 11

$\begin{array}{ll}\text { Theoretical Overlap } & 15\end{array}$

$\begin{array}{ll}\text { Health Action Process Approach } & 17\end{array}$

Key Social-Cognitive Determinants of Health Behavior 19

$\begin{array}{lr}\text { Risk Perception } & 20\end{array}$

$\begin{array}{ll}\text { Outcome Expectancy } & 22\end{array}$

$\begin{array}{ll}\text { Self-Efficacy } & 23\end{array}$

$\begin{array}{ll}\text { Intentions } & 25\end{array}$

$\begin{array}{ll}\text { Empirical Application of the HAPA } & 28\end{array}$ 


\section{METHOD}

Participants

Description of Participants $\quad 46$

$\begin{array}{ll}\text { Study Sites } & 47\end{array}$

Procedures

$\begin{array}{ll}\text { Study Approval } & 48\end{array}$

$\begin{array}{ll}\text { Participant Accrual and Retention } & 49\end{array}$

Data Collection Waves 51

Measures

Background Characteristics $\quad 53$

$\begin{array}{ll}\text { Social-Cognitive Measures } & 56\end{array}$

$\begin{array}{ll}\text { Physical Activity } & 63\end{array}$

Data Analysis

$\begin{array}{ll}\text { Descriptive Analyses } & 67\end{array}$

$\begin{array}{ll}\text { Hypothesis Testing } & 67\end{array}$

$\begin{array}{ll}\text { Sample Size Calculation } & 70\end{array}$

\section{RESULTS}

Participant Characteristics

$\begin{array}{ll}\text { Response Rates and Study Duration } & 74\end{array}$

$\begin{array}{ll}\text { Characteristics of Completers versus Non-Completers } & 76\end{array}$ 
Data Completion among Study Measures

Participant Characteristics of Completers

Socio-Demographic Differences among Study Measures

Diabetes-Related Characteristics

BMI and Physical Activity Characteristics

Psychological Characteristics

Hypothesis Testing

DISCUSSION

Summary of Results

$\begin{array}{ll}\text { Study Overview } & 102\end{array}$

Summary and Interpretation of Primary Findings 104

$\begin{array}{ll}\text { Summary and Interpretation of Ancillary Findings } & 127\end{array}$

$\begin{array}{ll}\text { General Conclusions and Implications } & 137\end{array}$

$\begin{array}{ll}\text { Physical Activity and DSME } & 137\end{array}$

Social-Cognitive Factors in DSME Physical Activity Initiation 140

HAPA Framework Applied to DSME and Type 2 Diabetes 144

General Strengths and Limitations 146

$\begin{array}{ll}\text { Final Summary } & 151\end{array}$

$\begin{array}{ll}\text { REFERENCES } & 182\end{array}$

APPENDIX

$\begin{array}{ll}\text { Study Measures } & 196\end{array}$

$\begin{array}{ll}\text { CURRICULUM VITA } & 227\end{array}$ 


\section{LIST OF TABLES}

TABLE $\quad$ PAGE

1. Factors that Influence Initiation \& Maintenance of Physical Activity 153

2. Overlap of Key Social-Cognitive Constructs Across Health Behavior 154 Models

3. Data Collection Sites with Sample Size and Diabetes Education Class 155 Format

4. Data Collection Waves, Timeline, \& Construct Measurement 156

5. Psychological \& Physical Activity Study Measures with Associated 157 Constructs

6. Summary of Reliability Statistics on Study Measures 158

7. Summary of Recommended Classifications of Physical Activity 160

8. Socio-Demographic Characteristics of Participants (Completers Only) 161

9. Percentage Comparison of Study Sample to Local, State, and National 164 Socio-Demographic Characteristics

10. BMI, Depressive Symptomology, and Diabetes-Related Characteristics 165 of Participants

11. Participant Leisure-Time Physical Activity Characteristics - Continuous Scores of Energy Expenditure

12. Participant Levels of Leisure-Time Physical Activity 168

13. Social-Cognitive Characteristics of Participants 169

14. Differences between Depression Status and Social-Cognitive Factors $\quad 170$

15. Differences between Annual Household Income and Social-Cognitive 171 Factors 
16. Intercorrelations between $\mathrm{H} 2$ Dependent Variable, Independent Predictors and Covariates

17. H2 Regression Coefficients, Standard Errors, $\beta$ Weights, and Confidence 173 Intervals for Model 2 (Full Model)

18. Intercorrelations between H3 Dependent Variable, Independent

Predictors, and Covariates

19. H3 Unstandardized Logistic Regression Coefficients, Wald Statistics, Adjusted Odds Ratios, and Confidence Intervals for Model 2 (Full Model) 


\section{LIST OF FIGURES}

$\begin{array}{ll}\text { FIGURE } & \text { PAGE }\end{array}$

1. The Health Action Process Approach (basic model depicting behavior initiation).

2. Health Action Process Approach Motivational Stage.

3. Health Action Process Approach Volitional Stage.

4. Flow Chart of Study Recruitment (all sites).

5. Regression Coefficients of Linear Regression Test of HAPA Motivational Stage Constructs.

6. Adjusted Odds Ratios for Logistic Regression Test of HAPA Volitional Stage Constructs. 


\section{INTRODUCTION}

\section{Study Background, Purpose, and Rationale}

\section{Type 2 Diabetes Mellitus}

Prevalence, severity, and cost. Diabetes mellitus (diabetes) is a chronic metabolic disorder characterized by insulin-resistance and subsequent dysfunction in insulinsecretion that systemically degrades glucose utilization (American Diabetes Association, 2010). Of the three major forms of diabetes, type 2 (formerly called non-insulin dependent or adult-onset) accounts for approximately $90 \%$ to $95 \%$ of all diagnosed cases (Centers for Disease Control and Prevention, 2007). Untreated or poorly managed diabetes yields a host of chronic, debilitating complications including hypertension, heart disease, stroke, central and peripheral neuropathies, damage to the eyes and kidneys, impotence, and non-traumatic lower-limb amputation as well as increased risk and cooccurrence of psychological distress and major depression (American Diabetes Association, 2010; Calles-Escandon \& Cipolla, 2001; Fisher et al., 2007; Gerich, 2005; Lin et al., 2004; Lustman, Penckofer, \& Clouse, 2007; Rizvi, 2004).

National and global estimates of prevalence, including projections toward 2030 , suggest diabetes is a global pandemic. Recent population based reviews estimate approximately 26.8 million people living in the United States (U.S.) will have diabetes in 2010 with projections of 35.9 million people by 2030 , a substantial increase in current 
and projected estimates over several years ago, attributed mostly to improved methodologies for estimating disease prevalence (American Diabetes Association, 2003; American Diabetes Association, 2008; Cowie et al., 2006; Shaw, Sicree, \& Zimmet, 2010; Wild, Roglic, Green, Sicree, \& King, 2004). These revised estimates suggest the national prevalence rate for 2010 will be $12.3 \%$ of the total U.S. population. Within the state of Kentucky, 2009 Behavioral Risk Factor Surveillance System (BRFSS) estimates suggest that the prevalence of diagnosed cases of diabetes at both the state and Louisville-metro area were at $11.5 \%$ and $10.4 \%$ of the population, respectively. (Centers for Disease Control and Prevention, 2008; Centers for Disease Control and Prevention, 2009).

In the United States, revised estimates suggest the economic cost for diabetes nationally will be approximately $\$ 198$ billion in 2010 , with the U.S. accounting for $52.7 \%$ of the global expenditure on diabetes - by 2030 these adjusted costs will rise to over $\$ 264$ billion (Zhang et al., 2010). These costs are substantially higher than previous estimates, a difference attributed to increases in both medical care costs out-pacing inflation and improved methodologies for estimating both prevalence and cost of diabetes (American Diabetes Association, 2003; American Diabetes Association, 2008; Zhang et al., 2010).

A behaviorally-managed disease. Evidence from landmark randomized trials indicated that intensive glycemic control could help delay the onset and reduce the severity of diabetes-related complications, ultimately improving diabetes-related quality of life (American Diabetes Association, 2010; Blonde \& Karter, 2005; Diabetes Control and Complications Trial Research Group, 1993; Diabetes Prevention Program Research 
Group, 2002; Gerich, 2005; Lindstrom et al., 2003; U.K. Prospective Diabetes Study (UKPDS) Group, 1998). Glycemic control in patients with diabetes is achieved through a complex, life-long regimen of self-care behaviors. These include proper diet, physical activity, medication (including insulin when necessary) self-monitoring of blood glucose (SMBG), as well as foot care, eye care, and routine clinic visits, making diabetes unique compared to other chronic diseases in which only one or two of these behaviors might be required (American Diabetes Association, 2010; King \& Glasgow, 2003).

Implementation of these self-care behaviors can be draining physically, cognitively, and emotionally for patients living with diabetes, leading to the additional risk of psychological-distress and co-occurring psychological disorders such as major depression, which can in-turn affect successful engagement in these self-care behaviors (Lin et al., 2004). Successful management of diabetes is heavily dependent on the individual patient to initiate and maintain these self-care behaviors. Of these, physical activity represents a unique challenge: a growing body of empirical literature suggests that physical activity plays a vital role in the prevention and delay of developing type 2 diabetes, as well as delaying the onset and severity of complications in patients with diabetes, yet it remains one of the most difficult behaviors to adopt and maintain.

\section{Physical Activity and Type 2 Diabetes.}

Benefits of physical activity. The benefits of engaging in regular physical activity are well documented in the empirical literature and highlight physical activity's importance in both the primary and secondary prevention and treatment of chronic illnesses including type 2 diabetes (American Diabetes Association, 2002; American Diabetes Association, 2010; Blue, 2007; Chipkin, Klugh, \& Chasan-Taber, 2001; Marcus 
et al., 2000; Sherwood \& Jeffery, 2000; Wing et al., 2001). Within the diabetes population, randomized controlled trials have demonstrated that both moderate and vigorous engagement in physical activity can both delay the onset of type 2 diabetes (for those in pre-diabetes metabolic syndrome) as well as delay the onset of diabetes relatedcomplications in those already diagnosed with diabetes (Diabetes Control and Complications Trial Research Group, 1993; Diabetes Prevention Program Research Group, 2002; Laatikainen et al., 2007; Lindstrom et al., 2003; U.K. Prospective Diabetes Study (UKPDS) Group, 1998). Over the past decade, researchers have uncovered mechanisms by which physical activity appears to delay diabetes-related complications. Both aerobic and strength-based physical activity appear to increase insulin-sensitivity at the cellular level resulting in greater glucose uptake and subsequently, lowered circulating plasma glucose in the bloodstream (Pedersen \& Saltin, 2006). Mounting evidence suggests that even a single bout of moderate- to-vigorous physical activity (aerobic or strength training) can result in more efficient glucose uptake and utilization for up to 24 hours (Chipkin et al., 2001; Pedersen \& Saltin, 2006).

Due to its beneficial acute and chronic effects, regular physical activity is prescribed to patients with diabetes as part of the cornerstone of treatment, which it shares with self-monitoring of blood glucose and proper diet. Yet, despite the known benefits of physical activity, sedentary lifestyles and limited regular physical activity continue to flourish. Estimates from the 2009 BRFSS data found that within the state of Kentucky, approximately $54.3 \%$ of the general population do not meet recommended amounts of physical activity (defined as 30 minutes of moderate-intensity activity, five or more days a week or 20 minutes of vigorous-intensity activity, three or more days per 
week), a figure slightly above the national median rate of $49.3 \%$ (Centers for Disease Control and Prevention, 2009). While this is an improvement over 2001 estimates of physical activity and on course with the Healthy People 2010 objectives, it remains clear that improving rates of physical activity among both the general and diabetes populations remains a public health priority. Understanding the determinants of physical activity is one approach to addressing this issue.

Determinants of physical activity. The past several decades of empirical research has yielded biological, sociological, psychological, and environmental factors that seem to influence the adoption or initiation and maintenance of physical activity in both the general and chronic illness populations. Table 1 (p. 153) highlights the number of factors that can help or hinder the successful initiation and maintenance of physical activity. In the person with diabetes, additional disease-specific complications can intensify the difficulty in engaging in physical activity (American Diabetes Association, 2010; Chipkin et al., 2001; Marcus et al., 2000; Pedersen \& Saltin, 2006; Schwarzer, 2008; Sherwood \& Jeffery, 2000; Thomas, Alder, \& Leese, 2004; Wing et al., 2001).

There are a number of both immutable and changeable factors that influence engagement in physical activity. Those factors that are resistant to change (e.g., gender, age, ethnicity, socio-economic status) do play an important role in identifying high-risk populations that may not be physically active. Marcus et al.'s (2000) comprehensive review identified that sedentary behavior was more prevalent among women, older adults, lower socio-economic status (low income/low education), and minorities. Factors that are more amenable to change (time, access, stress, health-risk profile, socialcognitive processes) have become the target of empirical research and clinical 
intervention in both the general and chronic disease populations (Schwarzer, 2008; Wing et al., 2001). In most cases, these factors are viewed as changeable and influence motivation and decision-making to engage in physical activity. Within the diabetes population, the addition of diabetes-specific complications as well as co-occurring difficulties (e.g., major depression, chronic pain) serve as important predictors of engagement (or lack thereof) in regular physical activity (Krein, Heisler, Piette, Makki, \& Kerr, 2005; Lin et al., 2004; Sherwood \& Jeffery, 2000). The severity and progression of diabetes-specific complications highlight the importance of accessing this population early in treatment to prescribe and promote recommended amounts of physical activity to help delay the onset of complications. Before discussing the recommendations for how patients with diabetes are presented this information, it is important to distinguish the determinants of physical activity within the context of initiation versus maintenance.

While the terms initiation and maintenance are used heavily in the physical activity literature, there remains no clear "golden rule" that distinguishes one from the other. Traditionally, when an individual meets the recommended guidelines for regular physical activity (discussed below) for six months, the individual is said to be in a maintenance phase (Marcus et al., 2000). While this six month time frame is frequently used in the literature and is the hallmark for the Maintenance stage in the Transtheoretical Model (Prochaska \& DiClemente, 1984), the six month period remains a point of contention among theorists in that it appears more arbitrary than grounded empirically as a key benchmark to identify someone who is maintaining a behavior (Armitage \& Conner, 2000; Schwarzer, 2008). Nevertheless, understanding the factors that influence continuation of a behavior such as physical activity remains a public health priority. Of 
note, reports of adherence to regular physical activity among patients with diabetes range from $19 \%$ to $33 \%$ (McNabb, 1997; Thomas et al., 2004; Walker \& Usher, 2003).

Conversely, since approximately $50 \%$ of the American adult population (and $60 \%$ to $80 \%$ of the American adult diabetes population) is not reaching the recommended amount of physical activity, the role of initiation also remains a key target for study and intervention (Centers for Disease Control and Prevention, 2009; Kirk, Mutrie, MacIntyre, \& Fisher, 2003; Marcus et al., 2000).

Recommended amount of physical activity. Current Centers for Disease Control and Prevention (CDC), American College of Sports Medicine (ACSM), and American Diabetes Association (ADA) physical activity guidelines for patients with type 2 diabetes is a minimum of 30 minutes of moderate-intensity physical activity, five or more days a week, for a total minimum recommended amount of 150 minutes/week of activity (American Diabetes Association, 2010; Donnelly et al., 2009; Kirk et al., 2003; Pedersen \& Saltin, 2006). In terms of energy expenditure, this is equivalent to 600 moderate METminutes per week (METs: metabolic equivalents - a multiple of resting metabolic rate) (Craig et al., 2003; Kirk et al., 2003). Patients with diabetes who are predominantly sedentary are typically advised to consider engaging in 10-minute bouts of activity for a minimum of 30 accumulated minutes of moderate-intensity activity a day (American Association of Diabetes Educators, 2009b). However, a recent ACSM position statement revision based on systematic reviews of physical activity literature recognized both a dose-response to physical activity (i.e., the more the better), and that sustained activity greater than 30 minutes may be necessary to achieve the health benefits of physical activity, suggesting that accumulated activity may not be enough (Donnelly et al., 2009). 
Patients with type 2 diabetes learn of these recommendations from several sources that serve to communicate and reinforce the importance of engaging in regular physical activity. These sources include their physician, diabetes educator, and diabetes selfmanagement education classes.

Diabetes self-management education. Diabetes self-management education (DSME) is the cornerstone for delivering instruction in diabetes self-care behaviors and providing the initial resources necessary to successfully self-manage diabetes (American Association of Diabetes Educators, 2009a; Jack, 2003). Common across all DSME courses is a basic core set of education designed to provide knowledge related to a number of behaviors essential in successful self-management. Nationally standardized curriculum developed by the American Association of Diabetes Educators (AADE) is delivered in classroom settings. This teaching typically spans approximately $8-10$ hours and covers topics on managing blood glucose, nutrition, physical activity, and basic problem-solving for the management of stress and diabetes-specific complications (Diedrich, Munroe, \& Romano, 2010; Funnell et al., 2010; Jack, 2003). A relatively recent paradigm shift in DSME occurred over the past decade from growing concerns regarding the effectiveness of DSME. This led to the development of the AADE-7 core measures of outcome performance designed to establish benchmarks across seven domains of diabetes self-care behaviors, including physical activity (Funnell et al., 2010; Mulcahy et al., 2003).

While DSME has generally demonstrated effectiveness in teaching nutritional and blood glucose monitoring skills, evidence in support of DSME influencing engagement in physical activity has generally been variable. A systematic review of nine randomized 
controlled trials revealed four studies that documented increases in physical activity among DSME participants, five studies showed no changes, and across all nine studies, it remained unclear what factors may have accounted for any demonstrated change (Norris, Engelgau, \& Venkat-Narayan, 2001). One of the primary reasons for these mixed findings is believed to be the limited amount of time available to diabetes educators to teach the required curriculum. Despite recognized importance of physical activity, it is generally not economical to focus DSME on physical activity due to the complexities related to this specific behavior change in contrast to other behaviors or topics that can be discussed (Diedrich et al., 2010). Another reason is a general uncertainty in the DSME literature as to what accounts for changes in physical activity patterns among patients attending DSME (American Association of Diabetes Educators, 2009a).

\section{Section Summary}

So why does it remain difficult for patients with type 2 diabetes to implement physical activity recommendations taught in DSME? Simply, for someone with diabetes, the barriers for engaging in regular physical activity are high and the benefits are distal when compared to the proximal feedback patients receive from engaging in less-intensive self-care behaviors such as checking one's blood sugar or receiving an eye or foot exam every few months. Due to the dynamic nature of this problem, health science professions approach this challenge from a number of different perspectives. One such perspective has been the focus of health psychology over the past several decades. Namely, to identify the social-cognitive factors (i.e., attitudes, beliefs, and behaviors) that shape motivation and enable decision-making to engage in physical activity. To understand the 
value of this approach, it is critical to examine the current understanding of key socialcognitive factors that function in both predicting and changing behavior.

\section{Social-Cognitive Models of Health Behavior}

Within health psychology, through the use of expectancy-based models predominantly grounded in social-cognitive theory, researchers have over the past several decades attempted to identify psychological factors that may play vital roles in the initiation and maintenance of health behaviors such as physical activity. Through both theoretical and atheoretical empirical exploration, social-cognitive factors (SCFs) have been identified that are both predictive of behavior and are malleable via intervention to influence behavior change (Armitage \& Conner, 2000; Conner, 2008; Lippke \& Ziegelmann, 2008; Maddux, 1993; Schwarzer, 2008). The health behavior models that will be briefly described here represent the foundation of theoretically-driven research over the past several decades. A brief overview of these different models will highlight both similarities and differences among these models and serve to introduce the underlying social-cognitive determinants predictive of physical activity.

This section seeks to help the reader understand that despite such a rich volume of empirical research, the prediction of health behavior continues to revolve around a select core of stage- and continuum (non-stage) based models. These models attempt to differentiate themselves in the causal explanation of behavior but are in fact wrought with overlapping constructs. As such, the empirical literature has yielded little headway as to which health behavior model best captures the causal processes of health behavior change; essentially no "gold-standard" model has surfaced. This section will conclude highlighting that despite this dilemma, more recent conceptualizations of the underlying 
SCFs through the Health Action Process Approach may help move the field closer to understanding what factors may be most important when examining social-cognitive predictors of physical activity.

\section{Health Behavior Models}

Continuum vs. Stage-based models. Health behavior models over the past several decades have collapsed into two separate categories: continuum or non-stage based models (e.g., Health Belief Model, Theory of Planned Behavior, Protection Motivation Theory) and stage based models (the most salient being the Transtheoretical Model) (Armitage \& Conner, 2000; Schwarzer, 1992; Schwarzer, 2008). A brief overview of these models will highlight the similarities and differences of these models and help elucidate why no single model has claimed prominence over all other models of health behavior. These models are presented within the context of type 2 diabetes and physical activity initiation as the targeted outcome behavior.

The Health Belief Model (Rosenstock, 1974) posits that a health behavior regimen is likely to be adopted when a threat of disease (or disease complications) is recognized through external cues and the perceived benefits of engaging in healthful behaviors to counter the threat outweigh the perceived barriers of such behaviors. The Health Belief Model (HBM) is a continuum model that incorporates several constructs that move a person with diabetes toward an increased probability of engaging in physical activity. Perceived threat is theorized to comprise of two factors: perceived susceptibility (or vulnerability) to a disease or complications and perceived severity to a disease or complications. Cues to action are viewed as external, such as receiving information from a healthcare provider, family member, or through DSME, that contribute to the 
individual's recognition of the perceived threat (Glanz, Rimer, \& Lewis, 2002).

Perceived benefits minus perceived barriers reflects a cost-benefit analysis framed in general statements (as opposed to if-then statements seen in outcome expectancies), such as "exercise would be good for me; exercise would help me better control my blood sugar," or "exercise would be difficult" (Schwarzer, 1992). The HBM is one of the few social-cognition models that explicitly recognizes the modifying role of sociopsychological factors (e.g., dispositional optimism, conscientiousness), sociodemographic factors (e.g., age, gender, and ethnicity), and illness-specific factors (e.g., diabetes severity) (Christensen, 2002). Based on the HBM, targets for clinical intervention to encourage initiation and improve maintenance of physical activity in patients with diabetes would focus on increasing cues to threat, enhancing perceived benefits, and decreasing perceived barriers.

The Theory of Planned Behavior (TpB), a continuum model, posits that behavioral intention is the most proximal determinant of behavior change (Ajzen, 1988, 1991, 2002). The TpB theorizes that intention to engage in physical activity is determined by one's attitude towards the behavior, subjective norms (i.e., perceived social pressure [normative beliefs]) that an individual may experience to perform a behavior, and perceived behavioral control $(P B C)$, defined as the perceived ease or difficulty of performing a behavior (Ajzen, 1991, 2002). Attitude reflects a person's appraisal of importance related to a behavior and is influenced by behavioral beliefs, a construct similar to outcome expectancies (Schwarzer, 1992). The development of subjective norms would reflect the effect of normative beliefs passed on via a number of different sources including spouse/family, physician, diabetes educator, DSME class, etc. (similar 
to cues to action in the Health Belief Model). Perceived Behavioral Control is theorized to predict behavior indirectly through behavioral intention in behaviors perceived to be under strong volitional control. In behaviors where volitional control is less clear or problematic, $\mathrm{PBC}$ is theorized to directly predict behavior (Armitage \& Conner, 2000; Schwarzer, 2008).

Protection Motivation Theory (PMT) is a continuum model that posits engagement in a healthful behavior (e.g., physical activity) or disengagement from an unhealthful behavior (e.g., sedentary lifestyle, smoking) is dependent upon an individual's appraisal of threat (i.e., what type of threat exists, what will happen if the behavior is not engaged or disengaged) and coping (i.e., does the individual posses the cognitive, affective, behavioral, and environmental resources necessary to address this problem) (Rogers, 1983). For the person living with type 2 diabetes, threat appraisal comprises the perceived vulnerability and severity of diabetes-related complications as well as what is termed "maladaptive response rewards," that represent the individual's positive appraisal of sedentary behavior (Floyd, Prentice-Dunn, \& Rogers, 2000; Maddux, 1993). Increases in perceived vulnerability and severity will increase the likelihood of engaging in physical activity whereas increases in maladaptive response rewards will detract from the likelihood of taking action. Conversely, coping appraisal incorporates a trade-off between self-efficacy (discussed later, but in short, one's belief that s/he can successfully engage in a particular behavior), and response efficacy, which is the individual's appraisal of how effective a particular intervention (i.e., behavior) is in alleviating the threat. When self-efficacy and response efficacy are high, the person with type 2 diabetes would be predicted to engage in physical activity. However, this response 
is tempered with response cost, the individual's assessment of how effortful or difficult engagement in physical activity may be. According to the PMT, these processes of threat and coping appraisal together predict whether or not someone with type 2 diabetes would initiate and maintain recommended levels of physical activity.

The Transtheoretical Model (TTM) is a stage-based model that suggests acquisition of healthy behaviors (e.g., physical activity) and cessation of unhealthy behaviors occurs in a dynamic process along a series of stages (Prochaska \& DiClemente, 1984). These stages of change (SOC) are Precontemplation (no intention of engaging in physical activity), Contemplation (thinking about initiating physical activity within the next six months), Preparation (making small changes in behavior but still not meeting a behavioral change criterion), Action (meeting a criterion, usually within the past 6 months), and Maintenance (meeting a criterion for six months or longer). With respect to physical activity in type 2 diabetes, the behavioral criterion would be the recommended guidelines of physical activity as noted above. Each SOC is associated with certain cognitive-behavioral strategies known as processes of change, which a person uses within particular stages (Prochaska \& DiClemente, 1984). Transition across stages is generally viewed as linear, but can be dynamic with progression or relapse (Marshall \& Biddle, 2001). In addition, transition from one stage to the next is facilitated by self-efficacy and decisional balance, a decision-making construct in which one bases a decision to act by weighing the pros and cons of engaging in the behavior (Weinstein, Rothman, \& Sutton, 1998). 


\section{Theoretical Overlap}

While there are a number of other health behavior theories worth mentioning, the handful of social-cognitive models presented here reflects the predominant theories applied to health behavior across numerous behavior and population domains, including physical activity and diabetes, over the past thirty years. Despite decades of research, no specific social-cognitive model has taken center-stage to become the preferred model to explain physical activity behavior. As such, throughout the social-cognitive literature, two recurring themes observed by this author are evident: (a) the social-cognitive model selected for any given study or intervention is typically done so with no specific empirical reasoning for choosing one model over another, and (b) researchers often conclude studies by highlighting the previous point and suggesting that future studies should compare different models to determine which social-cognitive model would likely explain a greater proportion of variance in physical activity. With respect to this latter point, some theorists have argued that the failure of a superior model emerging is attributable to the lack of studies that compare models to each other (Armitage \& Conner, 2000; Schwarzer, 2008). Contrary to this, it has been proposed that such an approach is inherently flawed and futile simply because there is a considerable degree of overlap in social-cognitive constructs that form the foundation of these health behavior models (Maddux, 1993; Schwarzer, 1992; Schwarzer, 2008).

Areas of overlap. Highlighted in Table 2 (p. 154) are the overlapping constructs within their respective models that indicate the redundancies across these models and underscores a limited set of key social-cognitive constructs that underlie these models. These SCFs are risk perception, outcome expectancies, self-efficacy, and behavioral 
intention (Schwarzer, 2008). As noted in Table 2, elements of risk perception, comprised of internal (threat) and external (cues) processes, are described in these models both explicitly and implicitly. Aspects of perceived threat are observed explicitly in constructs of the Health Belief Model (perceived threat = perceived vulnerability $\mathrm{x}$ perceived severity) and Protection Motivation Theory (perceived threat), while implicit threat is assumed during the Contemplation phase of the Transtheoretical Model as an individual moves into a stage in which actual consideration of a health threat (e.g., physical inactivity) becomes more evident. External cues are explicitly described by models such as the Health Belief Model (cues to action) and the Theory of Planned Behavior (normative beliefs) (Maddux, 1993). Also noted in Table 2, outcome expectancies are evident throughout each of these social-cognitive models. Outcome expectancies are described as weighing the benefits versus barriers (Health Belief Model), decisional balance (i.e., weighing the pros and cons: Transtheoretical Model), behavioral beliefs that help shape attitudes toward a behavior (Theory of Planned Behavior), and response efficacy in which one judges the expected outcome of a given coping behavior (Protection Motivation Theory). Additionally, across all these models, self-efficacy has become a key determinant of behavior either by the model's design or by revision (Armitage \& Conner, 2000; Conner, 2008; Schwarzer, 1992; Schwarzer, 2008). Selfefficacy has been added to the Health Belief Model to increase predictive power and to the Transtheoretical Model to help explain stage transitions. A key construct of the Theory of Planned Behavior, perceived behavioral control has been identified as having a high degree of implicit overlap with self-efficacy. This is because self-efficacious beliefs in being able to engage in a behavior implies a degree of control to engage in that 
behavior (Ajzen, 2002; Armitage \& Conner, 2000; Bandura, 1997; Schwarzer, 1992). Finally, behavioral intention is both explicitly (Theory of Planned Behavior, Protection Motivation Theory) and implicitly (Health Belief Model, Transtheoretical Model) present among these models.

Addressing the overlap. Schwarzer (1992) noted these overlaps and proposed a parsimonious social-cognitive model that would highlight and remove the redundancies prevalent among other health behavior models. Schwarzer's Health Action Process Approach (HAPA), has steadily gained attention in its application to the study of physical activity across community and chronic illness populations in Europe. Recently the HAPA became the center of empirical discussion and debate regarding its causal structure and utility as a model of health behavior change (Conner, 2008; Schwarzer, 2008; Sutton, 2008; Velicer \& Prochaska, 2008).

\section{Health Action Process Approach}

Model description. The Health Action Process Approach (see Figure 1, p. 176) posits that health behavior change (e.g., engagement in physical activity) is a process that involves two predominant stages (motivational and volitional) and these stages serve different roles depending on the phase of behavior examined (e.g., initiation versus maintenance of a behavior). The present study's focus was on the initiation of physical activity, therefore the description of the HAPA was restricted to the processes theorized to function in the initiation of a health behavior. With this in mind, according to the HAPA's motivational stage (see Figure 2, p. 177), the formation of a behavioral intention to engage in physical activity is dependent on three SCFs: risk perception, positive outcome expectancies for the specific behavior, and task-specific self-efficacy (i.e., the 
optimistic self-belief that one can engage in a specific activity, such as walking 3 miles, 3 times a week). Of the three theorized predictors of a behavioral intention, Schwarzer argued that self-efficacy would account for most of the variance in behavioral intention (Schwarzer, 1992; Schwarzer et al., 2003; Schwarzer, 2008; Schwarzer \& Renner, 2000). The importance of risk perception and positive outcome expectancies to the formation of a behavioral intention may vary by the behavior being considered. For example, risk perceptions of an imminent, life-threatening illness (e.g., cancer) may play a greater role in the prediction of a behavioral intention to seek a medical screening or adhere to treatment recommendations, compared to behaviors (e.g., physical activity initiation) in which the negative health outcomes for failing to adopt a behavior are distal (Schwarzer, 1999).

Once a behavioral intention is formed, the individual moves into the volitional stage (see Figure 3, p. 178) where additional self-regulatory SCFs help translate intended behavior into action. The HAPA volitional stage formulation was designed to address the intention-behavior gap, the phenomenon demonstrated in the empirical literature of developing a behavioral intention, but failing to successfully enact and engage in the behavior (Conner, 2008; Lippke, Ziegelmann, \& Schwarzer, 2005; Schwarzer, 2008; Sniehotta, Scholz, \& Schwarzer, 2005; Sutton, 2008). In the volitional stage, engagement in physical activity is contingent on two additional factors (in addition to intention): (a) barrier self-efficacy (i.e., the optimistic self-belief that one can engage in a specific behavior in the presence of internal and environmental barriers such as boredom, fatigue, or bad weather), and (b) action planning, in which the specific circumstances of engaging 
in a behavior are planned out (e.g., when to perform the behavior, how to perform the behavior, where to perform the behavior, etc.).

\section{Section Summary}

Despite almost thirty years of health psychology research to examine socialcognitive factors that may predict or influence physical activity initiation and maintenance, the empirical literature remains clouded by redundant health behavior theories wrought with overlapping constructs. The Health Action Process Approach was formulated to highlight the dynamic nature of behavior change while reducing the redundancies observed in various health behavior models. While the HAPA has received a greater amount of empirical attention over the past decade to further understanding of physical activity initiation and maintenance in chronic disease populations, no studies were identified in which the HAPA was applied to the type 2 diabetes population. Only two studies were identified that utilized the HAPA framework in pre-diabetes populations. A closer examination of the key social-cognitive determinants underlying the HAPA model will highlight general methodological issues. Further, it will introduce the current literature supporting the usefulness of the HAPA as a framework in understanding physical activity initiation and inform efforts in promoting the adoption and maintenance of physical activity.

\section{Key Social-Cognitive Determinants of Health Behavior}

The Health Action Process Approach draws upon the same set of social-cognitive factors that underlie the predominant health behavior models. Greater scholarly attention and scrutiny of the HAPA model's formulation has occurred over the past few years. A growing body of literature has both criticized and praised the HAPA model's 
parsimonious approach to explaining behavior through its emphasis on a select set of social-cognitive constructs: risk perception, positive outcome expectancies, self-efficacy, goal and implementation intentions (behavioral intentions and action plans, respectively) (Conner, 2008; Schwarzer, 2008; Sutton, 2008; Velicer \& Prochaska, 2008). A closer look at each of these social-cognitive constructs within the HAPA is warranted to briefly highlight general definitional and methodological issues. This section will conclude with a summary of the empirical studies examining the HAPA formulation of these SCFs within the context of both physical activity and diabetes, revealing the limited but promising support for the HAPA model as a framework for informing physical activity interventions in diabetes populations.

\section{Risk Perception}

Definition. Risk perceptions (i.e., attitudes, beliefs, perceptions about possible harm) in health behavior models are typically restricted to two types of beliefs: perceived vulnerability (or susceptibility) to a threat and the perceived severity of a threat (Brewer, Weinstein, Cuite, \& Herrington, 2004; Brewer et al., 2007; Weinstein, 2000). Within the type 2 diabetes domain, the threat would entail diabetes-related complications that are inevitable if a patient fails to optimize glycemic control through self-care behaviors such as regular physical activity. Within the HAPA, risk perception entails a combination of perceived vulnerability and perceived severity, a formulation similar to the Health Belief Model and Protection Motivation Theory. A key difference in the HAPA formulation is that risk perception is theorized to predict the formation of a behavioral intention (in the motivation stage), rather than to directly predict actual behavior. This is a critical principle because while the empirical literature yields some support for risk perceptions 
predicting behavior, the observed relationships are consistently weak, suggesting that risk perception may be better predictor of intention rather than actual behavior (Armitage \& Conner, 2000; Brewer et al., 2004; Brewer et al., 2007; Conner, 2008; Schwarzer, 1992; Schwarzer, 2008; Weinstein, 2000).

Measurement / Methodological issues. There are two predominant methodological issues related to the measurement of risk perceptions. First, like most SCFs there is no "gold standard" instrument to measure perceived vulnerability and perceived severity. Measures are typically restricted to specific domains of risk (e.g., diabetes-related risks, cardiac-related risk, seat-belt use risks, etc.).

A second methodological issue involves an ongoing debate regarding how perceived vulnerability and perceived severity are combined to form the risk perception construct (sometimes also referred to as perceived threat) (Armitage \& Conner, 2000). The dispute among theorists relates to whether the two factors (vulnerability and severity) should be combined additively or multiplicatively. If combined additively, a higher score on one measure would independently yield some formation of risk perception, whereas a multiplicative combination (i.e., an interaction) would considerably change the outcome (i.e., a risk perception score would equal zero if one of the two constructs was zero). It has been argued that a multiplicative combination of vulnerability and severity makes the most intuitive sense when forming a risk perception (Division of Cancer Control and Population Sciences, 2008). However, due to the methodological limitations in finding significant interaction effects, especially among correlational studies which comprise most of the social-cognitive literature, the multiplicative combination of severity and vulnerability is often abandoned in favor of an additive combination (McClelland \& 
Judd, 1993; Weinstein, 2000). The HAPA's formulation of risk perception was consistent with other theorists that recognized the methodological limitations of a multiplicative combination of severity and vulnerability. As such, consistent with the HAPA, the present study's formulation of risk perception used an additive combination of perceived severity and perceived vulnerability ratings, described in greater detail below (Schwarzer et al., 2003).

\section{Outcome Expectancy}

Definition. Outcome expectancy has been characterized as a judgment of the probable consequences that a performed behavior may produce (Bandura, 1997). Outcome expectancies are viewed in terms of positive expectations that act as "incentives" to perform a behavior and negative expectations that act as "discentives" to perform a behavior (Bandura, 1997, p. 21; see also Williams, Anderson, and Winett, 2005). An example of a positive outcome expectancy with respect to physical activity and diabetes would entail a cognition such as, "if I exercise three times a week, I will have better control over my blood sugar." Likewise, a negative outcome expectancy from someone with diabetes peripheral neuropathy may entail a cognition such as, "if I walk 2 miles, I will experience a tremendous amount of pain." Within the HAPA, positive outcome expectancies work in conjunction with self-efficacy and risk perception to move someone closer to formulating a behavioral intention to engage in physical activity. While some theorists have argued the necessity of studying both positive and negative outcome expectancies when predicting behavior, the HAPA posits that measurement of positive outcome expectancies only are necessary as the theory was designed to model the formation of a behavioral intention. In other words, according to the HAPA, 
regardless of whether one is attempting to model engagement in a healthy behavior (e.g., adoption of physical activity) or disengagement from an unhealthy behavior (e.g., stop smoking), formation of a behavioral intention to change behavior would require positive outcome expectancies to form the behavioral intention. This approach does not negate the importance and recognition that negative outcome expectancies may (and typically are) also operating in the process of behavior change. However, the HAPA formulation argues against the measurement of negative outcome expectancies, characterizing such measurement as redundant as these would be implicit in someone with low positive outcome expectancies for a behavior (Schwarzer, 1992; Schwarzer, 2008; Williams et al., 2005).

Measurement / Methodological Issues. Like many social-cognitive factors, there is no "gold standard" instrument due to variability in the types of physical activity measured as well as the different types of populations sampled. These two issues have been a significant set-back to advancing the field in understanding how outcome expectancies for physical activity help predict actual behavior, requiring individual studies to develop new measures or borrow measures from specific populations (e.g., cardiac population) to apply to target populations of interest (e.g., diabetes) (Williams et al., 2005).

\section{Self-Efficacy}

Definition. Self-efficacy may be one of the most widely recognized constructs of personal agency in the social and health sciences literature. Self-efficacy (also referred to as efficacy expectations) is traditionally defined as a cognitive, optimistic self-belief regarding one's ability to organize and implement internal and external resources to 
pursue and fulfill goal-directed behavior (Bandura, 1997; Schwarzer, 1992). Implicit in the self-efficacy construct is the function of knowledge, such as diabetes-related knowledge, that instructs a person in how to perform tasks or engage in behaviors within the context of situational barriers (Allen, 2004). Self-efficacy is formulated from four sources of information: performance accomplishments (i.e., engagement in physical activity), vicarious experience (i.e., watching others perform the behavior), verbal persuasion (i.e., specific instruction - e.g., via DSME), and physiological feedback (i.e., from performing physical activity behaviors) (Bandura, 1997). Self-efficacy is typically conceptualized as domain-specific (e.g., diabetes) and behavior-specific (e.g., physical activity) (Bandura, 1997; Schwarzer, 1992).

With the Health Action Process Approach, two types of self-efficacy play a pivotal role in the prediction of behavior initiation: task and barrier self-efficacy (Scholz, Sniehotta, \& Schwarzer, 2005; Schwarzer, 1992; Schwarzer, 2008; Schwarzer \& Renner, 2000). Task (or action) self-efficacy reflects a person's confidence in performing the actual components of a given behavior, such as walking three miles a day, twice a week, and functions primarily during the motivational stage of behavior initiation. Barrier (or coping) self-efficacy reflects a person's confidence to perform a given behavior in the context of situational barriers, such as exercising twice a week when tired, and functions in the volitional phase of the HAPA for both the initiation and maintenance of behavior. The concept of stage or phase-specific self-efficacy (i.e., task and barrier) is not unique to the HAPA and was first proposed in the addiction literature as a means of highlighting that self-efficacious beliefs may function differently depending on where someone resides in a self-regulatory cycle (e.g., initiation, maintenance, relapse, recovery) 
(Marlatt, Baer, \& Quigley, 1995; Schwarzer et al., 2007). The HAPA model incorporates several variant self-efficacy constructs depending on whether the model is measuring initiation or maintenance behaviors. For example, for physical activity, barrier selfefficacy is typically replaced with what is termed as maintenance self-efficacy and/or recovery self-efficacy. These constructs reflect efficacy expectations someone experiences when challenged with maintaining a recurring behavior (such as adhering to physical activity recommendations) or when recovering from a relapse in which the maintained behavior is stalled or stopped altogether, and then attempts are made to reimplement the behavior (Lippke et al., 2005; Scholz et al., 2005; Schwarzer, 1992).

Measurement / Methodological Issues. Similar to other social-cognitive factors, no "gold standard" instrument exists to measure self-efficacy. Typically, due to the domain-specificity (e.g., physical activity, diabetes), there exists great variability in the types of self-efficacy measures observed in the physical activity and diabetes literature. Moreover, no self-efficacy measure found by this author in the diabetes literature differentiates between task and barrier self-efficacy. Many of the self-efficacy measures observed in the diabetes literature often yield only a global score of confidence in engaging in diabetes self-care behaviors, but fail to quantify one's level of confidence in engaging in specific self-care behaviors.

\section{Intentions}

Goal vs. Implementation Intentions. Goal intentions, also referred to as behavioral intentions (or intentions), have traditionally been formulated by continuum models (e.g., Theory of Planned Behavior, Protection Motivation Theory) as the most proximal predictor of behavior (Ajzen, 1991; Armitage \& Conner, 2000; Gollwitzer, 
1993; Schwarzer, 1992; Schwarzer, 2008). The empirical literature has yielded inconsistent results with respect to behavioral intention as the most proximal predictor of behavior, a phenomenon referred to as the intention-behavior gap (Garcia \& Mann, 2003; Gollwitzer, 1993; Schwarzer, 1992; Schwarzer, 2008; Sniehotta, Scholz, et al., 2005; Sutton, 2008; Ziegelmann, Luszczynska, Lippke, \& Schwarzer, 2007). Attempts to bridge this gap by theorists led to intentions being dichotomized into pre-decisional and postdecisional processes that may better account for the variance in behaviors such as physical activity. Traditionally, the pre-decisional, goal-directed intentions have been relabeled behavioral intention as defined in the motivational stage of the HAPA. The behavioral intention is essentially conceptualized as pre-action, where internal motivational forces, such as risk perception, positive outcome expectancies, and taskoriented self-efficacy help to formulate and refine one's desires into a clearly cognizant, goal-directed intention (Schwarzer, 1992; Schwarzer, 2008; Sniehotta, Scholz, et al., 2005). However, as the empirical literature suggests, successful engagement in any behavior such as physical activity, often requires more than good intentions to translate desire into action. Hence, the concept of implementation intentions, also referred to as action plans, has evolved over the past two decades to further develop health behavior theory to inform how to translate behavioral intentions into actions (Gollwitzer, 1993).

In contrast to behavioral intentions, action plans address how, when, where, with whom, and other planning cognitions to facilitate translation of intentions into action. These plans are theorized to represent cognitive conditional statements (e.g., if-then statements) that are, "assumed [once developed] to lead to an immediate behavioral response without much conscious awareness" (Reuter, Ziegelmann, Lippke, \& 
Schwarzer, 2009, p. 364 ). Schwarzer (2008) claimed that action plans in the postintentional, volitional stage of the HAPA serve to bridge the intention-behavior gap by serving as a mediator between behavioral intention and actual behavior. While a number of studies have found simple mediation effects of action plans between behavioral intention and actual behavior, recent reviews of the HAPA have criticized the current empirical literature. This was due to limited types of populations and data sets that have been tested, which are predominantly confined to Europe and a finite number of chronic illness populations (e.g., cardiac or orthopedic rehabilitation) (Conner, 2008; Sutton, 2008). While the addition of action plans explains a greater proportion of overall variance accounted for in physical activity behavior, its role as a mediator has varied by study and its role as a moderator between behavioral intention and behavior remains unknown (Conner, 2008; Sutton, 2008). Additionally, the HAPA model posits that there are different types of strategic planning that may serve different functions depending on whether the behavior is in an initiation or maintenance stage. Specifically, while action plans are theorized to play a significant role in the initiation of a behavior, coping plans, (i.e., action plans tailored to address identified barriers) may play a more important role during the maintenance, relapse, and recovery stages of a behavior (Schwarzer, 2008). While the focus of the present study was on physical activity initiation, thereby emphasizing the role of action plans in the HAPA, coping plans are mentioned here since this will be addressed in subsequent reviews of HAPA literature.

Measurement / Methodological Issues. Like most social-cognitive factors, the measurement of both behavioral intention and action plans varies with specific studies, behaviors, and populations. Additionally, measurement of action plans provides a unique 
challenge in that action plans are idiosyncratic in nature. While strategies can be provided to help in the formation of action plans, the specific details of when, how, and where a behavior will be engaged in is typically subjective. As such, while open-ended questionnaires may provide more qualitative information regarding one's action plans, the construct is typically measured quantitatively using Likert-scale measures specific to the target sample.

\section{Empirical Application of the HAPA}

With a more thorough understanding of the key social-cognitive factors formulated within the Health Action Process Approach, it is now possible to take a closer look at the available empirical support for the HAPA that has been published over the past decade.

HAPA and Physical Activity. The Health Action Process Approach is observed in the recent empirical literature as a framework for understanding both behavior initiation and maintenance for a number of different health behaviors including seat-belt use, breast cancer screening, prophylactic dental care, and physical activity (Schwarzer, 2008). Within the physical activity literature, the HAPA has been applied to a handful of studies across healthy populations (Schwarzer et al., 2007) and chronic illness populations, including orthopedic rehabilitation, cardiac rehabilitation, and pre-diabetes (Absetz et al., 2007; Laatikainen et al., 2007; Lippke, Wiedemann, Ziegelmann, Reuter, \& Schwarzer, 2009; Reuter et al., 2009; Scholz et al., 2005; Scholz, Schuz, Ziegelmann, Lippke, \& Schwarzer, 2008; Schwarzer, Luszczynska, Ziegelmann, Scholz, \& Lippke, 2008; Sniehotta, Schwarzer, Scholz, \& Schuz, 2005; Sniehotta, Scholz, et al., 2005; Sniehotta, Scholz, \& Schwarzer, 2006; Uutela et al., 2004; Wiedemann, Schuz, Sniehotta, Scholz, \& 
Schwarzer, 2009; Ziegelmann, Luszczynska, Lippke, \& Schwarzer, 2007). Most of the identified studies are based on analyses from chronic illness samples derived from Berlin, Germany, limiting the generalizability of findings and highlighting the need to examine the usefulness of the HAPA's framework in more diverse settings and samples. Studies are presented here in chronological order (earliest to latest) and subdivided into two groups: (a) studies pertaining to tests of the entire HAPA model followed by (b) studies that explore specific aspects of the HAPA model (e.g., planning, or self-efficacy), yet inform the literature on the predictive value of the HAPA as a theoretical framework.

In the first study, 484 cardiac rehabilitation patients $(78.9 \%$ men $)$ were recruited for a four-wave longitudinal study to assess the HAPA model's framework and to explore stage-specific self-efficacy beliefs in predicting physical activity engagement following completion of a structured cardiac rehabilitation program (Scholz et al., 2005). Study measures were administered in the second week of cardiac rehab, at 2 and 4 months, and again at 1-year follow-up. A series of regression analyses examined the prediction of behavioral intention and physical activity. In the first regression analysis, behavioral intention to engage in physical activity was regressed onto motivational stage factors (risk perception, positive outcome expectancies, and task self-efficacy), with all three constructs accounting for statistically significant variance and task self-efficacy contributing the most variance, as predicted by the HAPA model. In a series of additional regression analyses, physical activity at times 2,3 , and 4 ( 2 months, 4 months, and 1year, respectively) was regressed onto volitional stage constructs (behavioral intention, action planning, maintenance self-efficacy) as well as covariates/history variables (e.g., past physical activity). Results across several analyses highlighted that post-intentional 
(volitional) constructs (action planning, maintenance self-efficacy, and recovery selfefficacy) contributed unique, statistically significantly variance to the regression equations. These findings lend some of the initial support to the HAPA framework's unique contributions to health behavior theory - namely, (a) that self-efficacy is not a static, optimistic self-belief but rather is dynamic and may function differently depending on where along the behavior change continuum one resides, and (b) translating intentions into action requires a further step: development and utilization of planning strategies to implement intentions and achieve goals.

In a similar study, 307 coronary heart disease patients in cardiac rehabilitation (79.8\% men) who were predominantly physically inactive $(61.2 \%)$ were recruited for a three-wave longitudinal study to assess the HAPA model's framework in predicting physical activity engagement (Sniehotta, Scholz, et al., 2005). A baseline survey administered to participants during cardiac rehabilitation assessed HAPA motivational stage constructs. Time 2 measurements of HAPA volitional stage constructs (maintenance self-efficacy, action planning) was administered at two months following discharge from cardiac rehabilitation with the third and final measurement of physical activity four months following discharge. Several structural equation models (SEM) were tested to examine the HAPA's theoretical framework in the context of the study's data. Again, consistent with the HAPA formulation, the structural equation models fit the data well. The first SEM tested the motivational stage pathways and found that $65 \%$ of the variance in behavioral intention was explained by HAPA motivational constructs: task self-efficacy, positive outcome expectancies, and risk perception. This first SEM also found that $11 \%$ of the variance in physical activity was explained by behavioral intention. 
In the second SEM (testing the full HAPA model), $69 \%$ of the variance in behavioral intention was accounted for by the motivational stage constructs (risk perception, positive outcome expectancies, and task self-efficacy). The addition of action planning and maintenance self-efficacy to behavioral intention (i.e., the volitional stage constructs) accounted for a greater proportion of variance in physical activity (24\%) compared to the model without action planning and maintenance self-efficacy (11-13\%). Hence as theorized, in a large sample across several months, the HAPA model framework appeared to successfully account for a significant proportion of physical activity variance over time.

In a third study 365 healthy, physically active participants ( $81 \%$ women) were surveyed via the internet across two time points assessing physical activity and HAPA model constructs (Schwarzer et al., 2007). At baseline, HAPA motivational stage constructs were measured. Five weeks later, measures of HAPA volitional stage constructs (action planning, recovery self-efficacy) and physical activity in days and minutes of fitness over the past week were administered. Consistent with the HAPA formulation, the structural equation model predicting physical activity fit the data well with action planning and recovery self-efficacy mediating the relationship between behavioral intention and physical activity. Twenty-one percent of the variance in physical activity was explained jointly by action planning and recovery self-efficacy. Most paths were statistically significant with three exceptions: (a) the path between risk perception and intention, (b) the path between positive outcome expectancies and intention, and (c) the indirect path (simple mediation) between intention and physical activity (i.e., action planning did not mediate the relationship between intention and behavior). This latter 
finding was contrary to the HAPA's formulation of action planning, as it is theorized to mediate the relationship between intentions and behavior, which was not found.

In a fourth study, several structural equation models re-examining data from two cardiac rehabilitation samples and one orthopedic rehabilitation sample examined the HAPA framework as a model of exercise adherence (Schwarzer et al., 2008). In the first analysis $(N=353$ cardiac rehab patients), exercise adherence at four months was predicted from the complete HAPA model. The data fit the model well with all pathways in both the motivational and volitional stages of the HAPA presenting as statistically significant. Seventeen percent of the variance in action planning was accounted for by the combination of behavioral intention and recovery self-efficacy, while $14 \%$ of the variance in exercise adherence was explained by the combination of action planning and recovery self-efficacy. In the second analysis ( $N=114$ cardiac rehab patients), exercise adherence at eight months was again tested with the HAPA framework. The data fit the model well again with all pathways demonstrating statistical significance except risk perceptions. In this analysis, $21 \%$ of the variance in action planning was accounted for by the combination of recovery self-efficacy and behavioral intention. Thirty-nine percent of the variance in exercise adherence was explained by action planning and recovery selfefficacy. Finally, in the third analysis ( $N=368$ orthopedic rehab patients), exercise adherence at 12 months was tested using the HAPA framework. In this last test, the model again fit the data well with all paths demonstrating statistical significance except risk perceptions and positive outcome expectancies. In this analysis, $39 \%$ of the variance in action planning was accounted for by behavioral intentions and recovery self-efficacy, 
whereas $23 \%$ of the variance in exercise adherence was accounted for by action planning and recovery self-efficacy.

Across these studies, two major points can be emphasized. First, that while these surveys are correlational in nature and therefore limit causal explanations, the analyses highlight the importance of volitional-stage factors (e.g., action planning and stagespecific self-efficacy) as proximal predictors of behavior, in contrast to other continuum models, such as the Theory of Planned Behavior that relegates behavioral intention as the most proximal predictor of behavior. Additionally, these studies highlight a diminishing effect of motivational-stage constructs, such as risk perception and positive outcome expectancies, over time. This is consistent with the HAPA formulation in that factors important to the initiation of a health behavior may not be as vital to behaviors that become more routine, such as a maintained behavior. Again, this highlights the importance of stage-specific social-cognitive constructs, specifically self-efficacy and planning, as proposed in the HAPA framework (Scholz et al., 2005; Schwarzer, 1992; Schwarzer, 2008; Schwarzer \& Renner, 2000).

The next several studies presented examine the hypothesized role of action planning in the early stages of behavior initiation. The HAPA posits that as a behavioral becomes habitual, action plans may become less important over time in favor of coping planning. Coping plans are essentially action plans formulated specifically to cope with challenges faced in the continued execution of a given behavior such as physical activity in the face of changing barriers.

A longitudinal study in a sample of cardiac rehabilitation patients $(N=352 ; 79 \%$ men) tested the role of action and coping planning in physical exercise initiation and 
maintenance (Sniehotta, Schwarzer, et al., 2005). Study authors hypothesized that levels of planning (action and coping) would increase in the weeks following discharge from cardiac rehabilitation but that as time progressed, increases in coping planning would accelerate past action planning. Hypotheses were developed to be consistent with expected phase-specific differences in the HAPA framework suggested between the initiation and maintenance of physical activity. The study measured action and coping planning and physical activity across three time points - two weeks into cardiac rehabilitation, and follow ups at two and four months, respectively. Consistent with the hypotheses, increases were observed in both action planning, coping planning, and physical activity across all three waves; however, coping planning continued to increase between waves two and three while action planning diminished and was statistically nonsignificant. This finding was consistent with the HAPA model's formulation of the varying roles of action plans from initiation to maintenance of physical activity. Namely, action plans should function to move patients from motivational-stage cognitions to engagement in behavior. As experience with the behavior grows, the functional aspects of action plans may become routine. In other words, knowing when, where, and how to engage in a routine behavior would become less important in favor of coping planning, in which situational or contextual barriers may interfere with successful engagement in physical activity (Schwarzer, 2008; Sniehotta, Schwarzer, 2005).

In a similar longitudinal study utilizing a three-group intervention, action plans and coping plans were examined along with other HAPA constructs to predict engagement in physical activity in cardiac rehabilitation patients $(N=211,22 \%$ women) (Sniehotta et al., 2006). At baseline, motivational-stage HAPA constructs were measured 
and then participants were randomized into three groups: (a) action planning group where participants were encouraged to formulate up to three action plans regarding physical activity, (b) action and coping planning combined group that incorporated the method from group 1 in addition to creating three coping plans to address anticipated barriers, and (c) a control group. At follow-up 10-weeks later, behavioral intention and physical activity was measured. Findings indicated that the both experimental groups demonstrated statistically significant increases in physical activity initiation and maintenance when compared to the control group, with the combined group outperforming the action-planning only group.

A study conducted in an orthopedic rehabilitation population $(N=368 ; 62.2 \%$ women) yielded similar findings (Ziegelmann et al., 2007). This longitudinal study compared behavioral intentions to implementation intentions (in this study, action and coping plans were not explicitly distinguished) in predicting physical activity behavior across three waves of data collection (baseline with six month and 12 month follow ups). At six months, no difference was detected between behavioral and implementation intentions. However, at twelve months, both behavioral and implementation intentions were statistically significant in predicting physical activity, yet implementation intentions were clearly superior. These findings suggest that behavioral intention may influence behavior as far out as 1-year, implementation intentions (i.e., a volitional-stage SCF) will better account for behavioral self-regulation as a behavior is maintained.

An additional internet-based study of 354 healthy adults ( $81.4 \%$ women) was an expanded analysis of Schwarzer et al.'s (2007) longitudinal assessment of the HAPA framework and physical activity (Scholz et al., 2008). This particular study examined the 
role of action planning and coping planning to determine: (a) whether both types of planning at time 1 improved the prediction of vigorous physical activity at time 2, (b) whether action and coping planning would operate consistent with the HAPA formulation (i.e., action planning for behavior initiation, coping planning for behavior maintenance), and (c) whether intentions moderated the effects of planning on behavior. With respect to the study's first hypothesis, structural equation modeling revealed two findings contrary to the HAPA formulation: (a) prediction of behavioral intention was statistically significant for self-efficacy, but not for positive outcome expectancies or risk perception, and (b) prediction of physical activity was statistically significant for behavioral intention and coping planning, but not action planning. The study's second objective was tested using two-group nested models of initiators of physical activity versus maintainers of physical activity. Findings revealed that action planning was not a statistically significant predictor of physical activity among initiators, as hypothesized by the HAPA. Further, coping planning was only a statistically significant predictor of physical activity among maintainers, consistent with the HAPA formulation. Finally, the researchers examined whether behavioral intention moderated the planning-behavior relationship. Their findings supported moderated-mediation in which action planning was a statistically significant predictor of physical activity among initiators, but only in those participants with higher levels of behavioral intention. Likewise, among maintainers, behavioral intention moderated the relationship between coping planning and physical activity. In a similar study, statistical re-analyses of a previously published study (Sniehotta, Schwarzer, 2005) examined whether behavioral intention moderated the mediated effect of action planning on physical activity (moderated-mediation) in a 
sample ( $N=124,81.7 \%$ men) of cardiac rehabilitation patients (Wiedemann et al., 2009). The researchers used bootstrapping methods to first establish simple mediation as hypothesized by the HAPA model (i.e., action planning mediates the relationship between behavioral intention and physical activity). Then, regression analyses were conducted to establish a significant interaction effect of action planning and behavioral intention on physical activity, a key requirement to then proceed to establishing moderated-mediation. Finally, bootstrapping methods were used to test indirect (mediated) effects at different levels of the statistical interaction. Their findings supported a moderated-mediation hypothesis of these HAPA variables. Action planning mediated the relationship between behavioral intention and physical activity, but the strength of the mediated effect was moderated by levels of behavioral intention.

A three-year longitudinal study of orthopedic rehabilitation patients $(N=328$, $61.5 \%$ women) examined latent growth curves of behavioral intention, planning, and exercise to further understand changes in the HAPA constructs over time (Reuter et al., 2009). Planning for this study was not defined as either action or coping; however, the measure reported appeared to contain questions from each construct. Statistical analyses revealed that (a) there were initial increases of both intention and planning for six months which then leveled off for the remaining three years, and (b) a mediation latent growth curve (LGC) model of behavioral intention, planning, and physical activity was statistically significant. This LGC model supported the role of planning as a mediator longitudinally, with increases in intention leading to increases in planning, and then increases in behavior; the direct path of intention on behavior was not significant over time. While these findings lend support to the role of planning as a mediator between 
intention and physical activity over time, the study's failure to specifically define the type of planning limits its contribution to understanding the role of action and coping planning in the broader HAPA framework.

A final study examined the possible moderating role of self-efficacy on the intention-planning-behavior relationship in an on-line sample of healthy adults $(\mathrm{N}=812$, 74.4\% women) (Lippke et al., 2009). Measurements of the HAPA's volitional constructs (action plans, self-efficacy - type undefined) and physical activity were measured at two time points with behavioral intention measured at baseline only. Researchers tested moderated mediation to examine whether self-efficacy would moderate the mediating role of action planning between intention and physical activity. All paths were statistically significant: simple mediation effects revealed action planning partially mediated the relationship between intention and physical activity. Moreover, moderated mediation revealed that a significant interaction of self-efficacy by action planning did contribute to the prediction of time 2 physical activity. These findings indicated that action plans mediated the relationship between intention and physical activity behavior, but this mediation was stronger among those individuals with higher self-efficacy.

These latter studies (Lippke et al., 2009; Scholz et al., 2008; Wiedemann et al., 2009) highlight continued questions regarding the hypothesized mediator role of action planning. The HAPA formulation posits a simple mediation role of action planning between behavioral intention and physical activity (i.e., that action planning mediates the relationship between behavioral intention and behavior) (Baron \& Kenny, 1986; Schwarzer, 2008). However, inconsistent results in the current empirical literature suggest (a) that in some studies this relationship (i.e., simple mediation) has yet to be 
found, and (b) that this relationship may be more complex than simple mediation may reveal, highlighting the possibility of moderated-mediation. While this yields new possibilities, it also reveals unique challenges. For one, as the HAPA has only been studied in a restricted number of populations, it remains unclear if even simple mediation of action planning exists in others, such as the diabetes population. Further, while one study (Wiedemann et al., 2009) was able to establish moderated-mediation within a sample of 124 cardiac rehabilitation patients, other studies (Lippke et al., 2009; Scholz et al., 2008) that found these more complex relationships required large $(>300)$ to extremely large $(>800)$ samples often viewed as necessary to find significant real-world interaction effects, but pose a major logistical obstacle for many researchers (McClelland \& Judd, 1993).

HAPA and Diabetes. While the Health Action Process Approach has yet to be applied specifically in a type 2 diabetes population, it has served as a framework for two pivotal lifestyle modification interventions implemented in at-risk populations within the past several years in Finland and Australia (Absetz et al., 2007; Laatikainen et al., 2007; Uutela et al., 2004). These studies targeted populations at-risk for type 2 diabetes who possessed a high number of significant pre-diabetes physiological (e.g., elevated body mass index, body weight, increased glucose tolerance) and behavioral markers (poor diet, sedentary lifestyle) that predicted, at a minimum, a one-in-six chance of developing type 2 diabetes within the next ten years following recruitment (Uutela et al., 2004). The Finnish Diabetes Prevention Study added to the growing body of evidence that lifestyle behavior modification (including physical activity) could delay and prevent the onset of type 2 diabetes and led to the development of Finland's National Diabetes Prevention 
Program. Noting the difficulties of translating interventions administered in wellcontrolled trials to the real world setting, Absetz et al. (2007) designed the Good Ageing in Lahti Region (GOAL) Lifestyle Implementation Trial to evaluate the effectiveness of implementing these interventions in a real-world setting. The lifestyle intervention sought to achieve five primary outcomes to delay or prevent type 2 diabetes - four dietary outcomes and one physical activity outcome: engagement in 30 minutes of moderate physical activity daily (Uutela et al., 2004). The intervention was structured on the HAPA model's theoretical framework and was split into six 2-hour group sessions delivered over the span of eight months. The first two sessions delivered information to increase behavioral intention to engage in diet and physical activity behavior by targeting the theorized HAPA constructs: risk perception, positive outcome expectancies, and task self-efficacy (i.e., targeted the motivation stage of the HAPA). The interventions used to target these constructs included self-monitoring of diet and physical activity, provision of information and discussion/evaluation of previous experiences with the targeted behaviors to activate self-efficacious thoughts. The latter four sessions focused on the volitional stage of the HAPA model. This predominantly targeted translating intentions into action via planning strategies, teaching how to overcome barriers (targeting barrier self-efficacy) and coping with relapse and maintenance issues. All six sessions were delivered in primary care settings across Finland by public health nurses, diabetes education nurses, and physiotherapists. One year follow-up data for the Finnish GOAL study $(N=352)$ found significant reductions in a number of anthropomorphic measures including diastolic blood pressure, weight, body mass index (men only), and waist circumference (Absetz et al., 2007). A significant difference was observed between 
baseline and one year follow-up among those participants engaging in 30-minutes of moderate physical activity. The largest increase in frequency was among those already meeting this objective at baseline. Overall, those meeting the physical activity objective at the end of the study were still less active compared to participants in the Finnish Diabetes Prevention Study. Nevertheless, the results of this GOAL study indicated that implementation of a lifestyle-modification intervention based on the theoretical framework of the HAPA could realistically and successfully be applied in a real-world setting.

Similar findings were observed in an equivalent lifestyle modification intervention to prevent type 2 diabetes undertaken in Australia. The Greater Green Triangle (GGT) Diabetes Prevention Project utilized the same Finnish-based intervention, implementing the Health Action Process Approach as a framework for changing health behavior in the prevention of type 2 diabetes (Laatikainen et al., 2007). The GGT trial ( $N$ =237) was implemented in Australian primary care centers using the same eight-month protocol with measurements at baseline, three months, and at 12 months. At the one-year follow-up, participants showed improvements across most targeted physiological markers (e.g., cholesterol, triglycerides, plasma glucose following 2-hour oral glucose challenge, diastolic blood pressure, weight and waist reduction), the exception being systolic blood pressure. Similar to the Finnish GOAL study, the majority of participants demonstrated improvements across most of these outcomes at the three month measurement and the effects persisted to 12 months.

The Finnish GOAL and Australian GGT trials add to the growing body of evidence in support of lifestyle behavior modification, such as physical activity, in 
reducing the incidence and/or delaying the onset of type 2 diabetes. Moreover, these findings demonstrated the potential usefulness of and growing interest in using the Health Action Process Approach as a framework for understanding how to predict and modify physical activity behavior in a pre-diabetes population. Prior to the present study, no literature was found using the HAPA in a type 2 diabetes population.

\section{Section Summary}

While support for the Health Action Process Approach is limited, the model's theoretical framework highlights the key social-cognitive factors important in the initiation and maintenance of physical activity. With respect to physical activity initiation in type 2 diabetes, the HAPA would posit factors such as risk perception, positive outcome expectancies, task self-efficacy, and behavioral intention would likely respond to instructional material presented in DSME. As with the two diabetes prevention studies that applied the HAPA framework (Absetz et al., 2007; Laatikainen et al., 2007), patients in DSME are in a unique position to receive physical activity recommendations. Further, the current HAPA literature on physical activity and pre-diabetes populations suggest the HAPA framework would be useful in examining the social-cognitive factors that may predict physical activity initiation in the DSME type 2 diabetes population.

\section{Study Purpose}

With the prevalence of type 2 diabetes increasing worldwide at an alarming rate, implementation of effective treatment interventions remains a public health priority. A growing body of literature suggests regular physical activity provides acute and longterm benefits in the delay of diabetes-related complications. People in Diabetes SelfManagement Education are in a unique position to receive accurate and appropriate 
information regarding the type, frequency, duration, and intensity of physical activity. Yet, the provision of information alone is not enough to encourage physical activity initiation. Social-cognitive factors, such as those theorized in the Health Action Process Approach, have been shown to predict engagement in healthy behavior, including physical activity. While early evidence suggests the HAPA model is a useful framework in understanding social-cognitive processes in physical activity initiation, less is known of its usefulness among type 2 diabetes populations, in particular those individuals currently in DSME. The purpose of the present study was to explore the relevance of the HAPA as a framework for understanding social-cognitive factors that may influence physical activity initiation among patients with type 2 diabetes participating in DSME.

\section{Specific Aims and Hypotheses}

The present study sought to address two primary aims. These aims are presented below followed by their respective hypotheses.
Aim 1
The present study's first aim was to examine changes among key social-cognitive factors among people with type 2 diabetes following presentation of recommended physical activity guidelines in Diabetes Self-Management Education. The social- cognitive factors that were expected to be most directly influenced by information presented in DSME comprise the motivational stage of the Health Action Process Approach: risk perception, positive outcome expectancies, task self-efficacy, and behavioral intention. Hypothesis 1 addressed this first aim.
Hypothesis 1 (H1): It was hypothesized that there would be a statistically significant increase in participants' scores on measures of (a) risk perception, (b) positive 
outcome expectancies, (c) task self-efficacy, and (d) behavioral intention, following presentation of DSME physical activity recommendations, when compared to baseline.

$\operatorname{Aim} 2$

The present study's second aim was to examine the relationships and causal pathways among key social-cognitive factors, as theorized by the Health Action Process Approach, in the prediction of physical activity initiation following completion of Diabetes Self-Management Education. This aim sought to address three key questions. Each question is presented below with associated hypotheses that were tested. Hypotheses 2 through 5 addressed this second aim.

Question 1. Informed by the HAPA theory's conceptualization of a motivational stage, did risk perception, positive outcome expectancies, and task self-efficacy predict the formation of a behavioral intention to engage in physical activity after the DSME presentation of physical activity guidelines?

Hypothesis 2 (H2). Following presentation of DSME physical activity guidelines, it was hypothesized that the formation of a behavioral intention to engage in physical activity was predicted by the combination of (a) risk perception, (b) positive outcome expectancies, and (c) task self-efficacy, after controlling for potential covariates. Identification and selection of potential covariates are discussed below under Data Analysis.

Question 2. Informed by the HAPA theory's conceptualization of a volitional stage, did behavioral intention, barrier self-efficacy, and action planning predict engagement in physical activity (defined below in Method section) after the presentation of DSME physical activity guidelines? 
Hypothesis 3 (H3): Specifically, it was hypothesized that the combination of (a) behavioral intention, (b) barrier self-efficacy, and (c) action planning would predict physical activity, after controlling for potential covariates.

Question 3. Noting the HAPA's conceptualization of action planning mediating the relationship between behavioral intention and behavior, did action planning mediate the behavioral intention - physical activity relationship? Further, was the relationship between behavioral intention and physical activity moderated by action planning?

Hypothesis 4 (H4): It was hypothesized that action planning would mediate the relationship between behavioral intention and physical activity as conceptualized by the HAPA.

Hypothesis 5 (H5): It was hypothesized that action planning would moderate the relationship between behavioral intention and physical activity engagement. 


\section{METHOD}

\section{Participants}

\section{Description of Participants}

All participants for this study were adults 21 years of age or older, diagnosed with type 2 diabetes mellitus, and currently attending Diabetes Self-Management Education classes offered by medical centers in the Louisville metropolitan and Southern Indiana areas.

Inclusion criteria. Study participants were screened via a self-report measure (discussed below) to meet the following inclusion eligibility criteria: (a) men or women with a current diagnosis of type 2 diabetes mellitus, (b) who were age 21 years and older, (c) were able to read, write, and understand English, (d) had access to an active, working telephone number for follow-up contact (including telephone-based data collection), and (e) were enrolled or planning to attend one of the DSME programs from area medical centers selected for this study (discussed below).

Exclusion criteria. Potential participants were excluded from this study if they met one of the following exclusion criteria: (a) individuals who were ever advised by their physician that they cannot participate in moderate or vigorous physical activity (b) were physically incapable of engaging in physical activity, due to physical impairment or

disability (e.g., amputation), (c) were undergoing medical treatment that could interfere 
with engagement in physical activity (e.g., participants undergoing dialysis or active treatment for cancer), or (d) were at baseline already participating in physical activity that exceeded the minimum recommended physical activity guideline of between 480 and 600 moderate MET-minutes per week, which is approximately 30 minutes of accumulated moderate physical activity per day, four to five days a week (Craig et al., 2003; Kirk et al., 2003). From the current study, 138 (19.2\%) of the 720 invited potential participants were determined ineligible based on these inclusion/exclusion criteria (see Figure 4, p. 179; discussed in greater detail below).

\section{Study Sites}

Site Selection. Participant recruitment targeted multiple DSME classes taught at medical centers in the Louisville metropolitan and southern Indiana region. The benefits of a multi-site approach were maximizing potential participant recruitment and accrual of a representative sample of the region's DSME population. The drawback to this approach was introduction of considerable variability across DSME programs. As a means to address this variability, sites were selected if the DSME program (a) used AADE-7 core curriculum derived from structured recommendations of the AADE and the ADA and (b) were taught by Certified Diabetes Educators. Six sites were identified as using the AADE/ADA core curriculum, despite differences in style presentation or number of sessions used to teach required curriculum.

In order to concisely refer to study sites throughout this paper, sites were labeled

"1 through 6," based on the chronological order in which recruitment proceeded (e.g., Site $1=$ Clark Memorial Hospital as this was the first site in which data was collected). Table 3 (p. 155) summarizes the DSME sites selected for this study, sample sizes of 
completed and non-completed cases per site, and class format for each site. The sample sizes reported for completed and non-completed participant cases per site are based on a total $\mathrm{N}$ of 210 individuals recruited at Wave 1 (e.g., baseline), as these are the cases for which site information was available (i.e., site specific information on the 278 nonresponders is not known because these individuals never returned study materials).

Class format and physical activity recommendations. Each DSME site had a preferred method of delivering DSME recommendations for physical activity. One site (Site 2) offered only single-session classes. At this site, all recommendations, including physical activity, were taught in this single session. Other sites (Sites 1, 4, 5, \& 6) tended to use a multi-session structure, in which classes were scattered over the course of several weeks (i.e., usually one class per week for four weeks). In multi-session classes, physical activity recommendations were provided during the second or third class. Site 6 also offered both single and multi-session classes to accommodate the needs of patients, and one site (Site 3) preferred individual meetings between a DSME instructor and patient. Due to DSME site variability in the timing of formal instruction of physical activity recommendations, each participant recruited in the study was tracked by their site and class type as a means to ensure follow-up measures were mailed to participants within an acceptable time frame (discussed below).

\section{Procedures}

\section{Study Approval}

Due to a multi-site study design, permission from Human Subjects Protection Committees / Institutional Review Boards (IRB) for each study site (with the exception of sites 3 and 5) as well as the University of Louisville was required to approve this 
study. Sites 3 and 5 did not have their own IRB and approved the study based on the IRB approval received from the University of Louisville. All sites waived requirements for separate, signed Informed Consent and granted a full HIPAA waiver due to this study's minimal risk design.

\section{Participant Accrual and Retention}

Recruitment. Recruitment approaches (in-person versus mail) for this study varied by individual DSME site, class format, and procedures approved by individual site's Institutional Review Boards. Overall duration of the participant recruitment phase of the study spanned 17-months. As noted in Table 3, Sites 1, 2, 4, and 6 provided most completed cases for this study and as participant accrual continued, efforts were directed towards these sites to optimize recruitment.

Single-session classes. For Site 2 and Site 6, approximately one week prior to the DSME class, this Investigator or the Research Assistant (RA) would bring sealed envelopes containing screening measures and study questionnaires (described below) to the site's Certified Diabetes Educator (CDE). The CDE would then display names and residential mailing addresses of potential participants attending upcoming single-session DSME classes to this Investigator or the RA, who would then transfer this information onto the sealed envelopes and then place these in the mail. The site would retain Personal Health Information (PHI), so neither the Investigator nor RA was ever in possession of PHI. If a potential participant was interested in participating, they were encouraged to follow the provided instructions of how to complete the screening measure and study questionnaires and mail back to this Investigator or the RA the completed study packet. Alternatively, potential participants could return the materials to the DSME class. When 
this occurred, the CDE requested the participant to seal his/her envelope, and would then either hold this until this Investigator or the RA visited again or would place in the mail which would send the packet to the Investigator. This method was also applied to Site 3 where individual classes were used.

Multi-session classes. For sites utilizing multi-session classes, individual DSME sites (sites $1,4,5, \& 6$ ) were interested in having this Investigator or RA recruit participants on-site. To remain consistent with the mailing procedures and adhere to IRB requirements, this Investigator or the RA would attend the first class of the multi-session class to recruit potential participants. At the beginning of the DSME class, this Investigator or the RA provided a brief overview of the study purpose, inclusion/exclusion criteria, risks/benefits, and compensation. Those individuals who were interested in participating or were unsure but wanted more time to think about it were issued a sealed study packet containing the screening measure and study questionnaires. They were encouraged to complete the packet as soon as possible and were asked to mail the materials back to this Investigator or the RA.

Retention. Due to the longitudinal design of this study and the increased possibility of subject attrition, participants were offered compensation for their participation. Each study Wave was accompanied by a grocery store gift card with a card value reflective of the level of effort that would be required of eligible participants. The gift card denominations were $\$ 15$ for Wave 1, $\$ 10$ for Wave 2, and $\$ 5$ for Wave 3, totaling $\$ 30$ for each participant who completed all three Waves. Gift cards were mailed to participants following completion of each study Wave. Additionally, as an added incentive to reduce attrition, eligible participants who completed all three Waves were 
eligible for one of two $\$ 100$ gift card lotteries. Lottery winners were selected using a random number generator and issued the gift cards.

\section{Data Collection Waves}

This study incorporated a longitudinal design, spanning approximately six to eight weeks from the presentation of physical activity recommendations, depending on a participant's respective DSME class format. This study's design objective was to capture data measurements at three time points, referred to as waves, consistent with measurement guidelines advocated by the AADE. The AADE's guidelines recommended assessment of physical activity at baseline, two to four weeks later, and then every three to six months. This time frame was believed to best ensure clinical intervention by a diabetes educator for problem-solving to resolve potential barriers to physical activity (Mulcahy et al., 2003). To accommodate the AADE Guidelines and multi-site DSME delivery format, the study design targeted gathering Wave 1 (baseline) data prior to presentation of the physical activity recommendations, Wave 2 approximately three weeks after the class in which participants received these recommendations and Wave 3 approximately three weeks after Wave 2 measurement.

In practice, noting variability in mailing times between study Waves, as well as differences of class presentation (e.g., single-session versus multi-session classes), a cutoff of eight weeks (56-days) after presentation of physical activity guidelines was used as the maximum amount of time a participant could participate in the study. In other words, after attending the class in which the physical activity guidelines were presented, participants must have completed the final Wave 3 measurement within eight weeks or $\mathrm{s} /$ he would be removed from the study. This timeframe was intended to account for 
mailing time variability while also keeping the study as close to the AADE recommendations as possible. To estimate time differences between Waves, means and standard deviations were calculated for those subjects who completed all Waves of the study $(N=152)$. The mean number of days between Wave 1 (baseline) and Wave 2 was 27.93 days $(S D=9.9$ days), while the mean number of days between Wave 2 and Wave 3 was 22.76 days $(S D=6.36)$. No participants who successfully completed the study went beyond eight weeks after receiving physical activity guidelines. Participants who went beyond their respective eight week cutoffs were considered non-completers. All three study Waves described below are summarized in Table 4 (p. 156).

Wave 1 (Baseline). Participants who were eligible and agreed to participate were instructed via a printed information sheet to complete the baseline questionnaire packet and mail it back to the Investigator or bring the packet to the next DSME class, where the sealed packet could be collected and mailed to the Investigator. The baseline packet questionnaire assessed socio-demographic background, diabetes history, baseline physical activity, and all construct measures of the Health Action Process Approach.

Wave 2. The second questionnaire packet was mailed to participants by this Investigator so that it could be completed by the participant within two to three weeks following presentation of physical activity recommendations. The Wave 2 questionnaire packet consisted of a repeated administration of the measures reflecting the HAPA constructs and physical activity.

Wave 3. At approximately three weeks after each participant completed his/her Wave 2 questionnaire packet, the Investigator contacted them by telephone to assess current physical activity. Additionally, participants were asked if they attended the 
DSME class and to describe in general the activity guidelines that were discussed as a means to verify attendance to that class. Originally, it was proposed that site attendance records would be reviewed to verify this; however, this would have affected the study's status of minimal risk and incur additional delays in study approval. Only two participants who were contacted for Wave 3 measurements indicated that they did not attend the class in which physical activity recommendations were presented. These subjects were reclassified as non-completers since they were not exposed to the physical activity guidelines.

\section{Measures}

A list of study measures and associated constructs can be found in Table 4 . The measures used in this study are described in detail below. Only participants who were considered "completers" (e.g., completed all three study Waves; $N=152$ ) contributed to reliability analyses of these measures. A brief summary of psychological and physical activity measures and their associated constructs are presented in Table 5 (p. 157). Internal consistencies for each Wave and test-retest reliabilities are presented in Table 6 (p. 158).

\section{Background Characteristics}

Screening questionnaire. This seven item, self-administered measure assessed potential participants regarding the study's inclusion/exclusion criteria. This screener queried age, type of diabetes, access to a working telephone, ability to read, write, and understand English. Additionally, the screener cued potential participants to identify (a) if s/he was unable to participate in any physical activity, or (b) if $s /$ he has been advised by their physician not to participate in moderate or vigorous physical activity. Finally, one 
question asked participants if s/he was already participating in the minimum level of recommended physical activity.

Socio-demographic questionnaire. Demographic and socio-economic characteristics were collected from each study participant using a 12 -item questionnaire. Sample items included age, gender, height/weight, race/ethnicity, educational level, marital status, employment status, occupation, and annual household income.

Diabetes history. Diabetes history was obtained from an abbreviated Diabetes History questionnaire developed by the University of Michigan's Michigan Diabetes Research and Training Center (Michigan Diabetes Research and Training Center, 1998). This questionnaire was developed in 1998 for population-based surveys within diabetes populations and assesses diabetes history across a number of domains including medication use, potential co-morbidities, and satisfaction with diabetes care. Two additional questions were added asking participants (a) to identify how long participants have been diagnosed with type 2 diabetes, and (b) if participants are currently taking insulin as part of their diabetes treatment.

Body Mass Index. For the present study, anthropomorphic measures were restricted to height (in inches) and weight (in pounds) in order to calculate body mass index (BMI) using the Imperial BMI formula and compare BMI values to the World Health Organization's Obesity classifications (World Health Organization, 2010). This classification system characterizes three levels of obesity: Class I (BMI 30.00 to 34.99 ), Class II (BMI 35.00 to 39.99), and Class III (BMI $\geq 40$ ). Additional classifications include Overweight or Pre-Obese (BMI 25.00 to 29.99), and Normal range (BMI 18.50 to 24.99). 
Depressive symptomology. A measure of depression was incorporated in this study as a possible covariate in the multivariate analyses, noting there is an extensive literature on how depressive symptomology may adversely affect diabetes selfmanagement (Fisher et al., 2007; Lin et al., 2004). Depressive symptomology was screened by the 10-item Center for Epidemiologic Studies Short Depression Scale (CESD 10) (Andresen, Malmgren, Carter, \& Patrick, 1994). The CES-D 10 screens for possible depressive symptoms over the previous seven days, yielding a total score of 30 with higher scores suggestive of depressive symptomology. The CES-D 10 has typically been used with cut-off scores of $\geq 8$ and $\geq 10$, yielding estimates of depression prevalence of $19.3 \%$ and $11.7 \%$, respectively (Andresen et al., 1994). While both cut-offs have been reported in the literature, a cut-off score of 10 has typically been used for screening purposes, and was selected for the present study (Center for Research on Women with Disabilities, 2009). This measure has predominantly been applied to older adult and chronic disease populations, but no studies were identified that used the CES-D 10 exclusively in the DSME population. Krein et al. (2005) used the CES-D 10 with a cut-off score of 10 in a sample of 993 veterans with chronic pain and diabetes; however reliability data was not reported. Carnethon et al. (2007) used both cut-offs ( $\geq 8$ and $\geq 10$ ) to predict the incidence of type 2 diabetes among older adults, but again reliability data was not reported. The CES-D 10 has demonstrated good internal consistency $(.85)$ in a predominantly female sample of 201 patients with multiple sclerosis (Harrison \& Stuifbergen, 2001). In the current study, the CES-D 10 demonstrated good internal consistency, Cronbach's $\alpha=.83(n=148)$. 
Mental status screen. A brief cognitive screener was used to ensure participants older than 60-years-old did not have impaired cognitive functioning to ensure the validity of the study responses. The 11-item Telephone Interview for Cognitive Status (TICS) takes approximately ten minutes to administer and has been demonstrated to have good psychometric properties (test-retest reliability $r=.90, p<.001$ ) and a sensitivity of 1.00 and specificity of . 83 when using a cut off of $<25$ in clinical populations (e.g., stroke patients) (Desmond, Tatemichi, \& Hanzawa, 1994). The TICS was administered to all participants aged 60 years and older $(n=49)$ during the Wave 3 telephone interview, taking approximately seven minutes to complete.

\section{Social-Cognitive Measures}

Risk perception. As noted previously, risk perception is the combination of severity and vulnerability ratings, and there remains much debate regarding either the multiplicative or additive combination of severity and vulnerability. To remain consistent with HAPA authors, risk perception for this study was computed as the additive combination of three scores: perceived severity of diabetes complications, perceived vulnerability to complications (self-ratings), and perceived vulnerability to complications (ratings of others) (Schwarzer et al., 2003). Severity was measured by the 18 -item Diabetes-Specific Health Beliefs questionnaire assessing beliefs about severity (DSHBSeverity) while vulnerability was measured by the 17 -item Diabetes Specific Health Beliefs questionnaire assessing beliefs about vulnerability (DSHB-Vulnerability) (Lewis \& Bradley, 1994). The DSHB-Severity measure queried participants to rate the seriousness of both diabetes-specific complications as well as general medical disorders. Two additional questions asked respondents to estimate perceived severity of their 
diabetes at the time of administration and in 10 years. Respondents reported severity using a five-point Likert scale: (0) indicates that the problem is not serious, (1) not serious enough to be worrying, (2) moderately serious, (3) very serious and (4) extremely serious. Additionally, each item has an option for participants to check a box indicating "not sure what problem is." The DSHB-Severity yields three separate (i.e., independent) scales based on different scoring conventions recommended by Lewis \& Bradley (1994), (a) an eight item perceived severity of complications, (b) an eight item perceived severity of general disorders, and (c) 2-item perceived severity of diabetes. Conversely, the two items of the last score subscale (perceived severity of diabetes) can each be treated as independent scores. The variability in scoring conventions proposed by Lewis \& Bradley was a result of the wide range in patient health literacy encountered in their reliability studies.

In the current study sample, 23 out of 152 participants on Wave 1 , and 14 out of 152 participants on Wave 2 endorsed "not sure what problem is," across individual complications questions on the DSHB-Severity measure, making it difficult to compute the 8-item scores. Per Lewis \& Bradley's (1994) scoring options, and recommendations from Schwarzer et al., (2003), Severity was defined by the single-item perceived severity of diabetes score, and was used as one of the scores to create the risk perception variable.

The DSHB-Vulnerability measure queried participants to rate perceived vulnerability to similar diabetes-specific complications and general disorders from the DSHB-Severity measure, but required respondents to do so from two different perspectives. First, participants were cued to consider an average person with similar characteristics (age, sex, treatment regimen, and average level of diabetes control) and 
then rate the likelihood of such an average person of developing the listed problems on a five-point Likert scale: (0) very unlikely, (1) quite unlikely, (2) neither likely nor unlikely, (3) quite likely, and (4) very likely. Then, participants were cued to make a second set of ratings using the same Likert scale from their own perspective (e.g., indicate how likely you feel it is that $y o u$ will develop the following problems). Lewis and Bradley (1994) indicated that the first set of ratings (of the average person) was incorporated to offset the possibility that participants may underestimate or deny their perceived vulnerability to the listed health risks either through ignorance of the specific disorder or through an optimistic bias that could skew personal estimates of vulnerability to diabetes-complications or general disorders. Lewis and Bradley argued that "personal risk-reducing strategies," such as engagement in self-care behaviors to improve blood glucose control through diet and exercise, may lead participants to underestimate perceived personal vulnerability to the queried diabetes-related complications (p. 262).

As with the DSHB-Severity measure, the DSHB-Vulnerability measure also allowed participants to check a box indicating "I already have this problem." Lewis \& Bradley (1994) noted in their experience that this option often resulted in a large amount of missing data across the individual items. For example, as participants endorsed a specific problem (i.e., indicated that $\mathrm{s} / \mathrm{he}$ had the particular disease), $\mathrm{s} / \mathrm{he}$ failed to provide vulnerability ratings (a phenomenon observed in the present study as well). Lewis \& Bradley noted that "listwise deletion" would result in too many cases being thrown out (p. 270). As such, they recommended using mean-replacement to handle missing data. Alternatively, the DSHB-Vulnerability measure incorporated two additional Likert-scale questions, asking participants to rate self and average person ratings of vulnerability for 
diabetes complications that could stand alone as separate measures of perceived vulnerability. Per Schwarzer et al., (2003), ratings from these two latter questions were used to complete the risk perception variable.

For the present study, risk perception then was the additive combination from perceived severity of diabetes complications, perceived vulnerability of complications (self-ratings), and perceived vulnerability of complications (ratings of others), yielding a three-item scale, ranging from 0 to 12 , with high scores indicating greater perceived risk (Schwarzer et al., 2003). This resulted in a risk perception score consistent with HAPA authors' conceptualization and consistent with scoring convention options proposed by Lewis \& Bradley (1994). In this present study, risk perception demonstrated adequate internal consistency (Wave 1: Cronbach's $\alpha=.66, n=150$; Wave 2: Cronbach's $\alpha=.74$, $n=152)$.

Positive outcome expectancies. Positive outcome expectancies for physical activity was measured by four items that comprise the exercise sub-scale from a 20 -item outcome expectancy scale used previously in two studies examining self-care behaviors (including physical activity) in participants with type 2 diabetes (Skelly, Marshall, Haughey, Davis, \& Dunford, 1995; Williams \& Bond, 2002). Participants were asked to rate their level of agreement on an 11-point scale ranging from (0) totally disagree to (100) totally agree on two positively-worded and two negatively-worded statements about outcomes in engaging in physical activity (e.g., I will get sore if I exercise). Scores from the two negatively-worded items were subtracted from 100, and then an average from all four items was obtained, with higher scores indicating more positive outcome expectancies for physical activity. Previously reported internal consistency in the 20-item 
measure was poor $(\alpha=.5)$, while test-retest reliability was adequate across a two week interval (.93) (Skelly et al., 1995). Among the four-item outcome expectancies scale, internal consistency was reported as .67 (M. J. Bond, personal communication, November 6,2007$)$. In the present study, this measure demonstrated internal consistency of Cronbach's $\alpha=.30$ at Wave 1 and Cronbach's $\alpha=.54$ at Wave 2 .

Task self-efficacy. Task self-efficacy or one's confidence in performing the actual components of a given behavior (e.g., duration, intensity, and frequency of physical activity), was measured using an adapted four-item instrument used in a cardiac rehabilitation population (Blanchard, Rodgers, Courneya, Daub, \& Blonde, 2002). Participants were asked to rate their confidence on a 10-point Likert scale ranging from (1) not at all confident to (10) very confident regarding their ability to exercise (or engage in physical activity) for 30 accumulated minutes at a moderate intensity 2 times a week, 3 times a week, 4 times a week, and 5 times a week. In a sample of 79 cardiac rehabilitation patients, average internal consistencies were good across four waves of data collection (average $\alpha=.92$ ). On this measure, higher scores suggested higher perceived confidence to engage in 30 minutes of physical activity on the respective days. The task self-efficacy measure's stem was adjusted for this study to reflect the physical activity recommendations of 150 minutes/week of moderate-intensity physical activity (Donnelly et al., 2009; Kirk et al., 2003; Pedersen \& Saltin, 2006). This measure demonstrated very good internal consistency, Cronbach's $\alpha=.94$ and .94 , at Waves 1 and 2, respectively. Behavioral intention. Behavioral intention was measured via two-items adapted from previous studies examining behavioral intentions in cardiac rehabilitation patients and undergraduate students using the Theory of Planned Behavior (Blanchard, Courneya, 
Rodgers, Daub, \& Knapik, 2002; Blanchard, Rodgers, et al., 2002). Adaptations of these items were based upon recommendations from Ajzen (1991) and were commonly used in research examining the Theory of Planned Behavior and exercise (Courneya \& McAuley, 1994). The first question asked participants: "My goal during the next three weeks is to engage in physical activity for 30 accumulated minutes at a moderate intensity which was then rated on a seven point Likert scale with responses ranging from 1 (not at all) to 4 ( 3 days a week) to 7 (every day). The second question asked: "I intend to engage in physical activity for 30 accumulated minutes at a moderate intensity, three to five times per week, over the next three weeks," which was then rated on a seven point Likert scale with responses ranging from 1 (strongly disagree) to 7 (strongly agree). The two items were then averaged to yield a behavioral intention score, with higher scores indicating greater intention. Variants of this format have yielded moderate to high correlations ( $r=.39$ to .60$)$ with self-reported physical activity in samples of both cardiac rehabilitation and undergraduate students (Blanchard, Courneya, et al., 2002; Blanchard, Rodgers, et al., 2002). In the current study, the behavioral intention score yielded adequate internal consistencies, Cronbach's $\alpha=.73$ and .81 for Waves 1 and 2, respectively.

Barrier self-efficacy. Barrier self-efficacy, or one's confidence to perform a given behavior in the context of situational barriers, was measured by the 12-item Barriers Efficacy Scale (McAuley \& Mihalko, 1998). This measures asked participants to rate their confidence in overcoming twelve common barriers to engaging in physical activity 3 times a week on an 11-point Likert scale ranging from $(0 \%)$ no confidence at all to (100\%) completely confident. Sample barriers included bad weather, boredom, 
pain/discomfort, vacation, etc. On this measure, higher scores indicated higher perceived confidence to overcome common physical activity barriers. This measure has demonstrated very good internal consistency across three time waves in a sample of 41 cardiac rehabilitation patients (Maddison \& Prapavessis, 2004). In the current study's sample, this measure demonstrated good internal consistency, Cronbach's $\alpha=.93$ at Wave 1 and .93 at Wave 2.

Action planning. Action planning for this study was measured by a selfadministered instrument with four response items that completed the statement stem, "I have made a detailed plan regarding..." For each response item, the respondent answered on a four-point Likert scale, ranging from (1) not at all true to (4) exactly true, rating how true each question was for that participant. For example, the participant was asked "I have made a detailed plan regarding... when to do my physical activity," and would respond by rating how true this was for her/him. Responses across all four items were averaged to yield a total continuous score of action planning, with higher scores indicating greater use of planning strategies (R. Schwarzer, personal communication, October 30,2007$)$. This measure of action planning has been used across several European-based studies examining the Health Action Process Approach and physical activity (Scholz et al., 2005; Schwarzer et al., 2007). In a sample of 484 coronary heart disease patients in Germany, the action planning measure had an average internal consistency of .93 across two waves of data collection (Scholz et al., 2005). In the present study's sample, this measure yielded good internal consistencies, Cronbach's $\alpha=.89$ at Wave 1 and Wave 2. 


\section{Physical Activity}

Physical activity measure. A self-report method for measuring physical activity was selected due to cost restrictions and study feasibility, despite known drawbacks in accuracy when compared to more objective measures of physical activity such as accelerometers and pedometers (Sallis \& Saelens, 2000; Tudor-Locke \& Myers, 2001). The International Physical Activity Questionnaire - Long Form and LongForm/Telephone (IPAQ-L and IPAQ-L/T, respectively) were selected to measure physical activity. The IPAQ-L, used for Wave 1 and 2 measurements, is a selfadministered, 27-item questionnaire that assesses physical activity over the past seven days across several domains including job-related physical activity, transportation-based physical activity, home-based (e.g., housework, yard work) physical activity, leisure-time physical activity, and sedentary behavior. The IPAQ-L/T, used for the Wave 3 telephone follow-up, is an identical instrument to the IPAQ-L with 27-items structured for telephone-based administration. The IPAQ- $\mathrm{L}$ and $\mathrm{L} / \mathrm{T}$ measure both habitual and leisuretime physical activity and yield continuous and categorical scores.

Habitual versus leisure-time activity. The IPAQ was originally developed to address the lack of internationally comparable measures of habitual physical activity (Bauman et al., 2009; Craig et al., 2003; Sjostrom et al., 2005). Habitual physical activity has been defined as physical activity incorporating any movement of the large muscle groups that can be achieved either through more intensive activities (e.g., cycling, running, walking), or more passive activities (e.g., gardening, job-related activities) (Kozakova et al., 2007). The impetus for this type of measurement was recognition that populations living in under-developed or developing nations engaged in physical activity 
throughout the day merely as a function of their daily activities and tasks, and that this activity was often unaccounted for by leisure-time measures of physical activity. The IPAQ-L and L/T measure habitual physical activity through the domains of job-related physical activity, transportation-based physical activity, and home-based (e.g., housework, yard work) physical activity. In contrast, leisure-time physical activity (LTPA) traditionally referred to as physical activity engaged in during one's leisure-time, such as planned physical activity (e.g., exercise), has been the primary self-reported measure of physical activity in the U.S. LTPA reflects the measure of physical activity that has traditionally been compared to recommended guidelines of 150 minutes of activity per week (Sjostrom et al., 2005). The IPAQ-L and L/T address LTPA through its leisure-time physical activity domain measure.

While habitual physical activity measurement is useful for international comparisons, LTPA was the predominant physical activity measurement of interest for the present study. This was due to several reasons. First, the U.S. population is predominantly sedentary across the habitual physical activity domains, noted by the nation's industrial focus (sedentary-based professions) as well as infrastructure (e.g., urban and rural construction that emphasizes reliance on automobile transportation). In the present study, approximately $75 \%$ of participants worked in sedentary professions. Second, guidelines and recommendations to increase physical activity presented in DSME avail themselves to more feasible behavior changes in the leisure time domain in contrast to other domains. In other words, it is more realistic to increase physical activity in one's free time in contrast to changing one's profession, method of travel, or homecare responsibilities in order to facilitate increased physical activity. Third, over the past 
several years the IPAQ's measurement of habitual physical activity has revealed the tendency for populations to significantly over-report activity across all domains (Bauman et al., 2009; Fillipas, Cicuttini, Holland, \& Cherry, 2010). While this is a recognized problem in self-report measures of physical activity in general, the IPAQ has been particularly susceptible to this due to the number of domains measured in characterizing habitual physical activity (B. E. Ainsworth, personal communication, May 19, 2010). As such, descriptive and hypothesis testing in the present study focused on the LTPA domain specifically, as this enabled comparisons with the CDC/ACSM (also referred to as DSME recommendations from this point forward) physical activity recommendations taught in DSME. This approach limited the likelihood of over-reporting associated with the IPAQ's habitual physical activity scores.

Continuous versus categorical scores. The IPAQ-L and L/T yield both continuous and categorical scores for characterizing physical activity (Sjostrom et al., 2005). The IPAQ's continuous scores for physical activity are characterized as energy expenditure, defined as MET-minutes per week (METs: metabolic equivalents - a multiple of resting metabolic rate). As noted above, due to severe over-reporting on the IPAQ, continuous scores can skew the distribution of physical activity scores. Moreover, continuous scores on the IPAQ can suggest engagement in the recommended amounts of physical activity when this may not be the case. For example, a participant reporting $\geq 600$ moderate MET-minutes per week based on continuous scores would appear to meet the minimum level of recommended physical activity. However, if the participant's activity occurred across two days in a week, this pattern of activity would likely not reflect the beneficial effects of regular moderate-intensity activity (i.e., the recommendation is to engage in 
moderate-intensity activity at least five days a week). As such, it has been recommended when using continuous scores that median MET-minutes per week be reported for descriptive purposes only, while categorical classifications of low, moderate, and high activity be used to both describe physical activity and for hypothesis testing (Bauman et al., 2009; Sjostrom et al., 2005). The categorical levels of activity are developed based on guidelines for scoring the IPAQ measures (Sjostrom et al., 2005). Definitions are presented in Table 7 (p. 160). One beneficial aspect of the IPAQ categorical scoring approach was reduction in the likelihood of misclassifying participants into higher levels of physical activity based solely on their continuous scores - this was accomplished by taking into account the number of days participants reported activity, per the IPAQ scoring conventions (Sjostrom et al., 2005).

Reliability and validity. The IPAQ-L has been utilized in over thirty international population-based studies and has yielded acceptable reliability. A reliability and validity study across twelve countries found test-retest Spearman's rho correlation coefficients ranging from .46 to .96 , with most around .80 and criterion validity coefficients around .30 , which while low, is consistent with other self-report physical activity measures (Johnson-Kozlow, Sallis, Gilpin, Rock, \& Pierce, 2006; Sallis \& Saelens, 2000). In the present study, the IPAQ-L demonstrated adequate internal consistency and test-retest reliability at Waves 1 and 2. Internal consistency was noted to be lower on the Wave 3 IPAQ-L/T form.

Physical activity defined. For the present study, physical activity was defined as a categorical variable that characterized participants' level of LTPA so it could be compared against physical activity guidelines taught in DSME. For descriptive purposes, 
this produced a three-level variable of LTPA, classifying participants into low, moderate, and high activity, as defined in Table 7 (Sjostrom et al., 2005). For hypothesis testing, this variable was converted into a binary dependent variable of physical activity participation, defined as 1 (participants who met the physical activity guidelines taught in DSME) and 0 (participants who did not meet the recommended physical activity guidelines taught in DSME). This approach was recommended to address extreme deviations from normality observed in the continuous IPAQ scores of this study's sample (B. E. Ainsworth, personal communication, May 19, 2010; P. Zahorik, personal communication, June 6, 2010).

\section{Data Analysis}

\section{Descriptive Analyses}

Appropriate descriptive statistics (e.g., mean, median, mode, standard deviation, frequency counts, etc.) were used to describe (a) socio-demographic characteristics (e.g., age, gender, ethnicity, etc.), (b) diabetes-related characteristics (e.g., duration diagnosed, insulin use, etc.), (c) BMI and physical activity characteristics, and (d) psychological characteristics (e.g., depressive symptomology, SCFs). Pearson chi-square tests and independent samples $t$-tests (with effect sizes) were used to assess differences between completers/non-completers as well as examine differences among completers.

\section{Hypothesis Testing}

Hypothesis 1 . H1 was tested using four paired-samples $t$-tests. Effect sizes (ES) for statistically significant $t$-tests were calculated using Cohen's $d$ for correlated samples

(Cohen, 1992; Dunlap, Cortina, Vaslow, \& Burke, 1996; Garson, 2008b). Consistent with 
Cohen's recommendations, effect sizes were defined as small (.20), medium (.50) and large (.80).

Hypothesis 2. $\mathrm{H} 2$ was tested using a single hierarchical multiple linear regression. The dependent variable (DV) behavioral intention at Wave 2 was regressed onto a set of Wave 2 independent variables (predictors) while controlling for the effects of covariates. Model specification (i.e., selection of independent predictors and covariates) was determined by a correlational analysis of variables guided by the HAPA theory and empirical evidence from the physical activity literature (Marcus et al., 2000; Schwarzer, 2008) . Pearson's $r$ and/or Spearman's rho were used, depending on the data type (e.g., interval versus ordinal/nominal) for the correlational analysis. Correlations with the DV identified as statistically significant $(p<.05)$ were entered into the regression equation. This approach to model specification helped to ensure that independent predictors and covariates were selected a priori based on the HAPA theory and empirical evidence, while also limiting inclusion of irrelevant causal variables that can inappropriately inflate $R^{2}$ and lead to suppression and/or spuriousness problems, confounding regression results (Cohen, Cohen, West, \& Aiken, 2003; Garson, 2010b). Consistent with established hierarchical regression techniques, covariates were entered into the regression equation at Block 1 (with categorical covariates dummy-coded), while independent predictors were entered at Block 2 (Garson, 2010b).

Hypothesis 3. H3 was tested using a single hierarchical logistic regression with the DV of physical activity participation defined as a binary of "1" meeting DSME recommended physical activity guidelines and " 0 " not meeting DSME recommended physical activity guidelines. The DV at Wave 3 was regressed onto a set of Wave 2 
independent variables (predictors) while controlling for the effects of covariates. Model specification was conducted consistent with the methods described above, with Spearman's rho used to evaluate correlations between the dichotomous DV and theoretically and empirically guided independent predictors and covariate variables (Garson, 2008a). Consistent with established hierarchical regression techniques, covariates were entered into the regression equation at Block 1, while independent predictors were entered at Block 2 (Garson, 2010b).

Hypothesis 4. $\mathrm{H} 4$ was tested using three statistical approaches to establish simple mediation (i.e., that action planning mediates the relationship between behavioral intention and physical activity as theorized by the HAPA). First, a series of multiple regression equations using methods described by Baron and Kenny (1986), adapted to logistic regression (Herr, 2010; MacKinnon \& Dwyer, 1993) were used to assess simple mediation. This process, referred to as causal steps, requires four conditions (i.e., statistical significance) to be met to establish simple mediation. These include: (a) path $c$ between predictor $\mathrm{X}$ (behavioral intention) and $\mathrm{Y}$ (the binary DV of physical activity); (b) path $a$ between the predictor X (behavioral intention) and the proposed mediator $\mathrm{M}$ (action planning); (c) path $b$ between the proposed mediator $\mathrm{M}$ (action planning) and $\mathrm{Y}$ (binary DV of physical activity); and (d) the path $c^{\prime}$ between predictor X on the DV (Y) is less (partial mediation) or zero (complete mediation) after controlling for the mediator, M (Baron \& Kenny, 1986).

The causal steps process has generally served to establish simple mediation in the empirical literature, but this process suffers from low power (Preacher, Rucker, \& Hayes, 2007). As such, two alternative statistical approaches that have become increasingly 
popular and supported in the empirical literature were also used to assess simple mediation: the Sobel $z$-statistic and the less conservative Bootstrapping method (Preacher \& Hayes, 2004; Preacher et al., 2007). The Sobel estimates effects (total, direct, and indirect) and yields a $z$-statistic with $p$-value to determine if the indirect (i.e., mediator) is statistically significant. The Bootstrap method allows random sampling of the available data based on a user-determined number of iterations (e.g., thousands of iterations possible due to computing speed in modern statistical software) to establish confidence intervals. The Bootstrap method is not a hypothesis test, but it is generally agreed that confidence intervals that do not span across "zero" is indicative of an indirect (i.e., mediator) effect (Preacher, 2009; Preacher, 2010).

Hypothesis 5. H5 was tested using a hierarchical logistic regression predicting the binary DV (as noted in $\mathrm{H} 3$ \& $\mathrm{H} 4$ ) with first-order predictors in Block 1 (behavioral intention, action planning), and the higher-order (interaction term) effect entered in Block 2. The interaction term of behavioral intention $\mathrm{x}$ action planning was created manually after centering scores for behavioral intention and action planning. Centering, or subtracting the sample mean from individual scores on all variables within the interaction term prior to computing the interaction term, is recommended to reduce the likelihood of multicollinearity (Cohen et al., 2003; Garson, 2010a).

\section{Sample Size Calculation}

The present study was originally powered to conduct the hierarchical linear regression of $\mathrm{H} 3$, which would have been the largest regression equation of the study with three independent predictors and up to seven possible covariates. With $\alpha=.05$, a power of .80 , and a medium effect size of $f^{2}=.15\left(R^{2}=.13\right)$, the required sample size 
was 118 completed cases (Buchner, Erdfelder, Faul, \& Lang, 2006). If this was adjusted to a more conservative effect size of $f^{2}=.11\left(R^{2}=.10\right)$, the number of needed complete cases increased to 158. Due to the extreme positive skew (i.e., non-normal distribution) of continuous scores on the IPAQ measure which seriously violated the assumption of normality in linear regression, this approach was abandoned in favor of logistic regression, as noted above.

With respect to logistic regression, the empirical literature notes that a large sample size is needed due to the use of Maximum Likelihood Estimation (MLE), albeit there is quite a lot of variability as to what constitutes a "large" sample (Cohen et al., 2003; Garson, 2010a). A series of simulation studies on logistic regression in the 1990s advocated that sample size be determined not by the total $N$, but by examining the limiting sample size, which is determined by examining the number of events versus nonevents (e.g., in binary logistic regression, an event $=1$ and non-event $=0$ ), and based on whichever is smaller (event or non-event), researchers were encouraged to ensure 10 to 15 events or non-events per variable (EPV) (i.e., 10 to 15 observations / participants per predictor variable) (Babyak, 2004; Peduzzi, Concato, Holford, \& Feinstein, 1995; Peduzzi, Concato, Kemper, Holford, \& Feinstein, 1996). This rule of thumb of a minimum of $10 \mathrm{EPVs}$ was found to be too conservative through a series of 2007 simulation studies (Vittinghoff \& McCulloch, 2007). Vittinghoff and McCulloch concluded that even 5 to 9 EPVs in logistic regression, often observed in epidemiology research, was suitable for interpretation and did not lead to the amount of bias originally reported by Peduzzi and associates. Moreover, Vittinghoff and McCulloch argued that 
while results based on less than $10 \mathrm{EPV}$ should be interpreted with caution, their findings did not justify disregarding results of studies using 5 to 9 EPVs.

In the present study, after examining the correlation analyses and listwise deletion of missing data for $\mathrm{H} 3$, the EPV was 9.6, based on a binary logistic regression equation of five predictors (three independent and two covariate factors) and a limiting sample size of 48 events (i.e., 48 cases classified as "1" versus 94 non-events "0"). This resulted in an EPV of 48/5 = 9.6 EPV, a suitable sample size based on Vittinghoff and McCulloch's simulations. As such, while the results of this study should be interpreted with caution, it appeared to have adequate sample size for logistic regression.

With respect to the moderation analysis in $\mathrm{H} 5$, it was recognized a priori that statistical tests of interaction effects with continuous variables are typically low powered in the real world (McClelland \& Judd, 1993). As such, it was quite likely no interaction effect would be detected in the present study.

Family-wise error. The family-wise error rate for hypothesis testing was set at 05 for this study, ensuring the risk of a type I error remained at 5\% throughout the study. A family was defined as a series of analyses or comparisons under simultaneous consideration, such as the case of multiple comparisons within the same hypothesis being tested (Benjamin \& Hochberg, 1995). In the present study, examples of families include $\mathrm{H} 1$, in which four paired-samples $t$-tests were conducted, or $\mathrm{H} 4$, in which three logistic regressions were tested. Bonferroni corrections for alpha were used when multiple tests were conducted within a "family." Corrections were not applied to the correlational analyses used for the selection of independent and covariate variables for $\mathrm{H} 2$ and $\mathrm{H} 3$ regression equations. This was because variables chosen for the correlational analyses 
were determined a priori based on theoretical and empirical findings. As such, inflated type I error rate is less of a concern in a priori analyses that are theoretically and empirically driven, as opposed to a post-hoc approach in which the actual risk of type I error would be higher if simply trying to find statistically significant relationships to enter into a regression equation (Garson, 2008a). 


\section{RESULTS}

\section{Participant Characteristics}

\section{Response Rates and Study Duration}

As noted in Figure 4 (p. 179), a total of 720 participants attending Diabetes SelfManagement Education classes across the Louisville-metro and Southern Indiana areas were invited to participate in this study. Of these, 94 participants $(13.1 \%$ of invited $)$ declined to participate and 138 participants (19.2\% of invited) were determined ineligible based on the inclusion/exclusion criteria. This resulted in 488 participants (68\% of invited) recruited to participate in this study. Of this, 278 participants $(57 \%$ of recruited) were non-responders, operationalized as patients who agreed to participate, were issued a study packet, but never returned the study packet with accompanying screening documents, making it difficult to determine the reason for not following through (i.e., it could not be determined how many of the non-responders declined to participate, were ineligible, or were simply not interested). The remaining 210 participants ( $43 \%$ of recruited or $29.2 \%$ of invited) returned completed Wave 1 study packets and were considered eligible participants for this study. Out of 210 participants who completed Wave 1, 183 participants ( $87.1 \%$ of Wave 1 participants) completed Wave 2 study packets, while 152 participants $(83.1 \%$ of Wave 2 participants or $72.4 \%$ of Wave 1 participants) completed Wave 3. Subsequently, the final sample of completed cases (i.e., 
"completer," defined as a participant who completed all three study Waves with useable data) was 152 participants ( $21.1 \%$ of invited).

Attrition. Attrition rates in this study were determined based on how many participants failed to complete this study with three Waves of usable data (i.e., "noncompleter," defined as a participant who entered the study by completing Wave 1 , but subsequently failed to complete all three study Waves with useable data). Using this convention, 27 participants (12.8\% of Wave 1) between Waves 1 and 2, and 31 participants (16.9\% of Wave 2) between Waves 2 and 3 dropped out of the study. Reasons for attrition varied. For most cases, attrition was due to either (a) failing to return a Wave 2 study packet in a timely fashion, thereby exceeding the eight-week time limit, or (b) being unreachable for the Wave 3 telephone call before the eight-week time limit, which was typically due to disconnected telephone lines or inability to make direct telephone contact with the participant. Aside from the two situations above, two participants dropped out due to medical reasons, two participants endorsed not attending the DSME class in which physical activity recommendations were taught, and one participant returned a Wave 2 study packet so late that the Wave 3 measurement was confined to less than a week's time after the Wave 2 measurement, bringing into question the validity of the results. Finally one participant was reclassified as non-completer after all three Waves of data were reviewed and determined to have a significant amount of missing data to prevent any analyses from being conducted (i.e., no useable data). Chisquare analyses yielded no statistically significant differences between study site and study completion status, indicating that attrition was not related to one or more particular study sites used in the present study. 
Study Duration. Data collection for this study spanned 17-months - from November, 2008, to April, 2010. An analysis of recruitment trends by season indicated most of the 210 recruited participants $(36.7 \%, n=77)$ were accrued during the summer of 2009 , with spring and fall 2009 equally obtaining 48 participants (22.9\%) each. Winter months yielded 37 participants (17.6\%). Since individual participation in the study spanned approximately two months, the variable season was determined by examining the dates of a participant's Wave 1 and Wave 3 measurements and if $\geq 75 \%$ of this window fell within a specific season, the participant was assigned to that season. Noting that seasonal changes could have affected completion of this study, a chi-square statistic was calculated to examine differences between completers $(n=152)$ and non-completers $(n=58)$ across seasons. Completers and non-completers did not significantly differ by season $\left(\chi^{2}=3.54, d f=3, N=210, p=.316\right)$.

\section{Characteristics of Completers versus Non-Completers}

As noted previously, "completers" $(n=152)$ were defined as participants who completed all three study Waves with useable data, while "non-completers" $(n=58)$ were defined as participants who entered the study by completing Wave 1 , but subsequently failed to complete all three study Waves with useable data. To evaluate differences between completers and non-completers, a series of chi-square statistical analyses were conducted on categorical scores to examine differences in socio-demographic, diabetesrelated, and physical activity characteristics. Additionally, independent-samples $t$-tests were conducted on continuous scores to examine differences between completers and non-completers on age, body mass index, depressive symptomology, duration of time since diabetes diagnosis, and social-cognitive variables. These analyses should be 
interpreted with caution noting that the sample size of completers was three times as large as the sample size of non-completers.

Categorical comparisons. There were no statistically significant differences between completers and non-completers on socio-demographic categorical variables (e.g., gender, ethnicity, marital status, education), with the exception of annual household income $\left(\chi^{2}=11.44, d f=5, n=199, p=.04\right)$. Examination of income classifications indicated most (48 out of 57) non-completers tended to be middle-class income households $(\$ 20,000$ to $\$ 100,000)$ with fewer cases below $\$ 20,000$, compared to completers. When looking exclusively at lower income participants (those with an annual household income $<\$ 20,000$ per year, 32 participants completed the study compared to 5 who did not. With respect to the other socio-demographic variables, the overall sample of completers and non-completers $(N=210)$ was predominantly women $(70.5 \%$ women compared to $29.5 \%$ men), Caucasian ( $85 \%$ Caucasian compared to $14 \%$ AfricanAmerican), married (58\%), and generally high school educated or higher (64.2\% posthigh school education).

There were no statistically significant differences related to study completion status and self-reported diabetes-related characteristics (e.g., insulin use, peripheral neuropathy, etc.). Insulin use among completers was $12.4 \%$ compared to $7.1 \%$ among non-completers. Among diabetes-related complications, the largest, albeit statistically non-significant difference, was noted with peripheral neuropathy, where $16.3 \%$ of completers versus $3.3 \%$ of non-completers reported having this co-morbid health condition. 
With respect to differences among physical activity characteristics, only Wave 1 comparisons could be examined, as up to half of non-completers (44\%) did not return a Wave 2 questionnaire or complete Wave 3 . There was no statistically significant difference in Wave 1 leisure-time physical activity levels (as defined in Table 7 between completers and non-completers. Most participants (completers and non-completers) fell in the low category of physical activity ( $82.4 \%$ for leisure-time physical activity).

Continuous comparisons. As noted, independent samples $t$-tests were used to examine differences between completers and non-completers on continuous variables. It should be noted that while this statistical test is often used for such comparisons, the comparison groups are not randomly assigned and therefore findings should be interpreted with caution (i.e., completers versus non-completers are not truly "independent" as they are coming from the same sample and were not randomized) (Garson, 2008b).

Even in the absence of a correction for type I error (e.g., Bonferroni correction which in this case would equal $.05 / 16$ comparisons $=.003$ ), there were no statistically significant differences between completers and non-completers among continuous scores for age, body mass index, duration (in months) of time since diabetes diagnosis, depressive symptoms, or most of the social-cognitive variables. The only two comparisons that were statistically significant (if not applying a correction for type I error) were Wave 1 measurements of barrier self-efficacy and action planning. Completers had higher Wave 1 barrier self-efficacy scores $(M=52.1, S D=22.4)$ compared to non-completers $(M=45.62, S D=18.5)$, a mean difference of $6.7(95 \%$ Confidence Interval [CI] of .17 to 13.2): $t=2.02, d f=208, p=.04$. The effect size (ES) 
using Cohen's $d$ for this difference was .28, a small effect. Completers also reported higher Wave 1 action planning scores $(M=2.57, S D=.93)$ compared to non-completers $(M=2.3, S D=.93)$, a mean difference of $.3(95 \% \mathrm{CI}$ of .02 to .6$), t=2.08, d f=208, p=$ $.04, \mathrm{ES}=.29$, again a small effect. If a correction for multiple comparisons was applied, these differences would be non-significant.

Overall, there were no statistically significant differences between completers and non-completers for this study. From this point forward, completers and "participants" may be used interchangeably, but refer only to those individuals who completed all three study Waves. All descriptive analyses, hypothesis tests, results presentations, and discussion will be limited to this final sample $(N=152)$.

\section{Data Completion among Study Measures}

All data for this study was manually entered, cleaned, and examined for outliers and statistical assumptions via descriptive statistics and graphical displays. Data processing and preparation also included examination and handling of missing data. As mentioned previously, the Diabetes-Specific Health Beliefs measures of severity and vulnerability (used to calculate the risk perception construct) had the most significant missing data issues. These were handled per recommendations from the measures' authors (Lewis \& Bradley, 1994; Schwarzer et al., 2003). With respect to nonstandardized questionnaires (e.g., all other social-cognitive measures), seven cases (i.e., participants) were identified with missing data on Wave 1 and 2 measures of task selfefficacy (one case on Wave 1), and barrier self-efficacy (three cases on Wave 1, four cases on Wave 2). None of these cases were missing more than $25 \%$ of the individual scale items (e.g., $>75 \%$ of the measures had completed data). To address this missing 
data, individual scores for each case with missing data were calculated using an adjusted denominator reflecting the total number of individual items with complete data. As noted, this correction was applied to a total of seven cases for barrier self-efficacy and one case for task self-efficacy. Missing data on the International Physical Activity Questionnaire IPAQ was addressed per scoring conventions recommended by the IPAQ Development Committee (Sjostrom et al., 2005). There was no missing data on the IPAQ's leisure-time physical activity scales across all three study Waves. No other study outcome measures were adjusted for missing data.

\section{Participant Characteristics of Completers}

The next several sections present participant characteristics $(N=152)$ organized into four domains for ease of review. These include (a) socio-demographic characteristics (e.g., age, gender, ethnicity, etc.), (b) diabetes-related characteristics (e.g., duration diagnosed, insulin use, etc.), (c) BMI and physical activity characteristics, and (d) psychological characteristics (e.g., depressive symptomology, social-cognitive variables).

Socio-demographic characteristics. Socio-demographic characteristics of this study's participants are reported in Table 8 (p. 161). Participants were predominantly middle-aged $(M=53.9$ years, $S D=11.6$ years $)$, women $(73.2 \%$, $n=109)$, and Caucasian $(82.6 \%, n=123)$. Approximately half were married $(53.1 \%, n=$ 78 ), and a third living with a partner/spouse $(34.9 \%, n=52)$. Most participants had a post-high school education (cumulative $61.4 \%, n=92$ ). This sample's annual household income was distributed across all socio-economic classes, with $22.6 \%(n=32)$ below $\$ 20,000,65.5 \%(n=93)$ between $\$ 20,000$ and $\$ 100,000$, and $12 \%(n=17)$ over $\$ 100,000$ annual household income. The median household income was approximately 
$\$ 39,999$. Most participants were either working full time $(44 \%, n=66)$ or retired $(22 \%, n$ $=33)$.

Comparisons with census data. Described in Table 9 (p. 164) are percentage comparisons of this study's sample to local (city and county), state (Kentucky), and national socio-demographic characteristics derived from the United States Census Bureau statistics from 2008 and 2000 (U.S. Census Bureau, 2010). Noting this sample was derived from both Louisville-metro and counties in Kentucky and Southern Indiana, comparable data was sought for comparison. The Census Bureau maintains data on Metropolitan Statistical Areas (MSA) that combines city and surrounding county information in order to better reflect geographical areas. However, data for the MSA that consists of the Louisville metropolitan area and surrounding counties, referred to as "Kentuckiana," was last obtained in the 2000 Census and had not yet been computed for the 2010 census. Therefore individual city and county statistics from 2008 from the Louisville-metro, Jefferson County, Kentucky, Clark County, Indiana, and Floyd County, Indiana were averaged together to yield a 2008 combined score for comparison with this study's sample. Comparable education data was last available in 2000.

As noted in Table 9, this study's sample was over-represented by women (73.2\%) in contrast to local, state, and national statistics. With respect to ethnicity, this study's sample was consistent with the combined local areas of approximately $80 \%$ Caucasians and $16 \%$ African-Americans. However, this study's sample under-represented Caucasians compared to state estimates but was consistent with national statistics. Conversely, African-Americans were over-represented when compared to state and national estimates. With respect to annual household income, this study's sample median 
income was slightly less than 2008 combined local area, state, and national statistics. This study's sample, when compared to the 2008 combined area estimates, suggests that participants in this study were comparable to the geographical region's (e.g., Kentuckiana) ethnicity and socio-economic status while over-representing women and possibly being more educated than state and national comparisons, at least based on 2000 census data for education.

Diabetes-related characteristics. Diabetes-related characteristics of this study's sample are reported in Table 10 (p. 165). Considerable variability was noted in the duration of time since participants had been diagnosed with type 2 diabetes mellitus, resulting in severely skewed data that affected the mean and standard deviation. This appeared to be the result of some participants who reported durations of up to 10 years. A review of other measures of central tendency revealed the median number of months diagnosed with type 2 diabetes was four months and the modal duration diagnosed was one month or less. A categorical score was calculated to better classify this sample which revealed $35.5 \%(n=54)$ were diagnosed one month or less while $64.5 \%(n=98)$ were diagnosed greater than one month. Most participants were not taking insulin $(82.9 \%, n=$ 126). Among co-occurring chronic illnesses and diabetes-related complications, $74.3 \%$ ( $n$ $=113)$ reported high blood pressure and $67.8 \%(n=103)$ reported high cholesterol. With respect to diabetes-related complications that could adversely influence engagement in physical activity, $22.5 \%(n=34)$ endorsed peripheral neuropathy.

BMI and physical activity characteristics. As noted in Table 10 (p. 165), the study sample's mean BMI $(n=144)$ was $36.5(S D=8.7)$. Most participants $(95.8 \%, n=$ 138) had BMI scores $\geq 25$ (overweight classification). Of these, $30.6 \%(n=44)$ were 
classified as Class III Obesity BMI according to the World Health Organization's ratings (World Health Organization, 2010).

Physical activity. Median leisure-time physical activity scores $(N=152)$ for walking, moderate-intensity, vigorous-intensity, and total leisure time physical activity are presented in Table 11 (p. 167). As the continuous scores have been subject to overreporting in previous studies, it was recommended by the IPAQ committee that categorical scores be used to describe physical activity levels and for inferential statistical analyses (Bauman et al., 2009; Sjostrom et al., 2005).

Categorical leisure time physical activity levels for the present study $(N=152)$ are presented in Table 12 (p. 168). At Wave 1, 85.5\% $(n=130)$ of participants engaged in a low-level of LTPA while $14.5 \%(n=22)$ of participants engaged in a moderate-level of LTPA. No participants reported a high-level of LTPA at Wave 1, indicating that no participants' self-reported physical activity exceeded the minimum amounts of recommended physical activity at baseline - a criterion for exclusion from the present study. In other words, at baseline only $14.5 \%$ of participants met the current DSME recommendations for activity, but did not exceed recommendations which would have invalidated their participation in the present study.

At Wave 2,78.3\% $(n=119)$ of participants engaged in a low-level of LTPA, $18.4 \%(n=28)$ engaged in a moderate-level of LTPA, and 3.3\% $(n=5)$ engaged in a high-level of LTPA. At Wave 3, 67.8\% $(n=103)$ engaged in a low-level of LTPA, $30.3 \%(n=46)$ engaged in a moderate-level of LTPA, and $2 \%(n=3)$ engaged in a highlevel of LTPA. As noted in Table 12, participants tended to migrate from a low-level to a moderate-level of LTPA across the course of the study. 
As discussed above, categorical LTPA scores at all three study Waves were collapsed to create the physical activity dependent variable for hypothesis testing, yielding a binary variable of meeting or not meeting DSME physical activity recommendations (also referred to as LTPA recommendations). Chi-square analyses were conducted to evaluate differences in meeting/not meeting the physical activity recommendations across the three study Waves. A statistically significant difference was found between Waves 1 and $2: \chi^{2}=8.53 ; d f=1 ; p=.003 ; n=152$, indicating more people $(21.7 \%, n=33)$ met recommendations at Wave 2 compared to Wave $1(14.5 \%, n$ $=22$ ). Additionally, a statistically significant difference was also found between Waves 2 and $3: \chi^{2}=22.90 ; d f=1 ; p<.001 ; n=152$, indicating more people $(32.2 \%, n=49)$ met recommendations at Wave 3 compared to Wave $2(21.7 \%, n=33)$.

Psychological characteristics. For the present study, psychological characteristics included cognitive status, depressive symptomology, and social-cognitive factors.

Cognitive status. Cognitive impairment screening of participants aged 60 and older using the TICS indicated that all of these participants met criteria for cognitively intact status: $M=37.4, S D=1.67, n=49$.

Depressive symptomology. The overall sample of participants with available depressive symptomology scores on the CES-D $10(n=149)$ yielded a mean $=9.77(S D$ $=6.01$ ). This continuous score was dichotomized (referred to as depression status) for descriptive purposes and analysis of differences among participants, using the recommended cutoff of 10 or higher to indicate possible depression on the CESD-10 (Krein et al., 2005). As noted in Table 10 (p. 165), depression status indicated over half 
of the participants in the present study were not depressed $(56.4 \%, n=85)$ compared to those participants who endorsed possible depressive symptoms $(43.6 \%, n=65)$.

Social-cognitive factors. The means and standard deviations for the socialcognitive measures are presented in Table 13 (p. 169). In Table 13 and below, results are reported as the names of the respective SCFs (as conceptualized by the HAPA model) rather than the name of the specific measures used, in order to simplify explanation of results. These SCFs included risk perception, positive outcome expectancies, task selfefficacy, behavioral intention, barrier self-efficacy, and action planning. All six SCFs were measured at Wave 1 and Wave 2 only.

\section{Socio-Demographic Differences among Study Measures}

A series of statistical analyses, using both chi-square and independent samples $t$ tests, were conducted to examine socio-demographic differences across the present study's primary outcome measures. Statistically significant differences $(p<.05)$ are summarized below, organized into three domains: (a) diabetes-related characteristics, (b) BMI and physical activity characteristics, and (c) psychological characteristics. These three domains were defined similarly to the descriptive domains described above. Only statistically significant differences among the present study's primary outcome measures within the three domains listed above were reported here. For example, statistically significant differences were observed among many socio-demographic characteristics (e.g., annual household income by ethnicity), but these were not reported as they were not the focus of the present study. 


\section{Diabetes-Related Characteristics}

With the exception of annual household income, there were no statistically significant socio-demographic differences between participants on diabetes-related characteristics. Insulin use by annual household income was statistically significant: $\chi^{2}=$ $4.36 ; d f=1 ; p=.04 ; n=142$, with more participants who endorsed using insulin with a reported annual household income of less than $\$ 39,000(12 \%, n=17)$ when compared to those participants who endorsed using insulin with a reported annual household income above $\$ 39,000(4.9 \%, n=7)$. Additionally, endorsed frequency of peripheral neuropathy (PN) by annual household income was statistically significant: $\chi^{2}=7.60 ; d f=1 ; p=.006$; $n=141$, with more participants who endorsed PN with a reported annual household income of less than $\$ 39,000(17 \%, n=24)$ when compared to those participants who endorsed PN with a reported annual household income above $\$ 39,000(6.4 \%, n=9)$.

\section{BMI and Physical Activity Characteristics}

Body Mass Index. A statistically significant difference was observed between participants with respect to age and BMI. A median split of age (participants 55 years and older versus participants 54 years and younger) was created solely for analysis of sociodemographic differences. An independent samples $t$-test of age was statistically significant for BMI: $t=-2.80 ; d f=141 ; p=.008$. Participants 55 years and older reported a lower BMI $(M=35, S D=7.3, n=76)$ compared to those participants 54 years and younger $(M=39, S D=10, n=67)$. The mean difference was $-3.8(95 \% \mathrm{CI}=-6.60$ to $.986)$ and the $\mathrm{ES}=.47$, a medium effect. Additionally, an independent samples $t$-test of depression status was statistically significant for BMI: $t=2.24 ; d f=139 ; p=.03$ Participants with probable depression reported higher BMI $(M=38.4, S D=8.54, n=62)$ 
compared to non-depressed participants $(M=35.12, S D=8.55, n=79)$. The mean difference was $3.24(95 \% \mathrm{CI}=.38$ to 6.11$)$ and the ES was .38 , approaching a medium effect size. No other statistically significant differences were observed with BMI, including physical activity, diabetes-related characteristics, or social-cognitive factors.

Physical activity. Socio-demographic differences were assessed using the binary DV of physical activity (e.g., meeting/not meeting recommendations). Only statistically significant differences for age and annual household income were identified.

Differences between age and physical activity. A statistically significant difference for age was found with Wave 2 LTPA: $\chi^{2}=4.55 ; d f=1 ; p=.03 ; n=148$. Among participants 55 years and older, only $15.4 \%$ of participants $(n=12)$ met LTPA recommendations compared to $84.6 \%$ of participants $(n=66)$ who did not meet LTPA recommendations. Among participants 54 years and younger, only $30 \%$ of participants ( $n$ $=21)$ met LTPA recommendations compared to $70 \%$ of participants $(n=49)$ who did not meet. No statistically significant differences for age were found with respect to Wave 1 or Wave 3 LTPA. These findings suggest that while general patterns of activity were the same between younger and older adults (e.g., more people in both groups did not meet LTPA recommendations compared to those who did meet recommendations), this proportion was greater in the older adult group.

Differences between annual household income and physical activity. With respect to annual household income, a statistically significant difference was found with Wave 2 and Wave 3 LTPA.

Wave 2 differences. For Wave 2, the difference was: $\chi^{2}=5.62 ; d f=1 ; p=.02 ; n$ $=142$. Among participants who reported an annual household income of $<\$ 39,000$ ( $n=$ 
73), only $15.1 \%$ of participants $(n=11)$ met LTPA recommendations when compared to $84.9 \%$ of participants $(n=62)$ who did not meet LTPA recommendations. This pattern was also observed for those participants who reported an annual household income > $\$ 39,000(n=69)$ : only $31.9 \%$ of participants $(n=22)$ met LTPA recommendations compared to $68.1 \%$ of participants $(n=47)$ who did not meet LTPA recommendations. Similar to the differences with age, the general pattern of activity between these groups was comparable, with most people not meeting LTPA recommendations. However, a greater proportion of people in the higher income group met LTPA recommendations compared to those in the lower income group.

Wave 3 differences. For Wave 3, the difference was: $\chi^{2}=7.42 ; d f=1 ; p=.006$; $n=142$. Among participants who reported an annual household income of $<\$ 39,000(n=$ 73 ), only $23.3 \%$ of participants ( $n=17$ ) met LTPA recommendations when compared to $76.7 \%$ of participants $(n=56)$ who did not meet LTPA recommendations. Again, this pattern was also observed for those participants who reported an annual household income $>\$ 39,000(n=69)$, with $44.9 \%$ of participants $(n=31)$ who met LTPA recommendations compared to $55.1 \%$ of participants $(n=38)$ who did not meet LTPA recommendations. These findings again suggested similar general patterns of physical activity between groups of lower and higher annual household income, with most people in both groups not meeting LTPA recommendations. However, these findings note that a greater proportion of individuals with higher annual household income met LTPA recommendations compared to those with lower self-reported annual household income. 


\section{Psychological Characteristics}

Depressive symptomology. Statistically significant differences, using independent samples $t$-tests, were found between depression status and all social-cognitive factors in the present study with exception of Wave 1 and 2 risk perception. These differences are summarized in Table 14 (p. 170).

Social-cognitive factors. Statistically significant differences were found between social-cognitive factors and gender, ethnicity, and annual household income. An independent samples $t$-test of gender was statistically significant for Wave 2 task selfefficacy: $t=-2.06 ; d f=147 ; p=.04$. Female participants reported less Wave 2 task selfefficacy $(M=5.53 ; S D=2.56 ; n=109)$ compared to male participants $(M=6.5 ; S D=2.469$; $n=40)$. The mean difference was $-.97(95 \% \mathrm{CI}=-1.9$ to -.04$)$ and the $\mathrm{ES}=.34$, a low-tomedium effect size.

With respect to ethnicity, a statistically significant difference was found with Wave 1 task self-efficacy: $t=2.13 ; d f=145 ; p=.04$. African-American participants scored lower on Wave 1 task self-efficacy $(M=4.68 ; S D=2.7 ; n=24)$ compared to Caucasian participants $(M=5.92 ; S D=2.61 ; n=123)$. The mean difference was $1.25(95 \%$ $\mathrm{CI}=.09$ to 2.4$)$ and the $\mathrm{ES}=.35$, a low-to-medium effect size. Noting that $83.3 \%(n=$ 20) of African-Americans in the present study were female, this finding may have been more related to differences in task self-efficacy between male and female participants rather than a difference due to ethnicity. This was evaluated with an additional set of independent samples $t$-test examining differences between Caucasian $(n=88)$ and African American $(n=20)$ females on Wave 1 and Wave 2 task self efficacy. Both analyses were statistically non-significant suggesting observed ethnic differences were 
indeed likely due to gender differences between women and men on task self-efficacy scores.

Similar to depression status, statistically significant differences, using independent samples $t$-tests, were found between annual household income and seven social-cognitive factors. These findings are summarized in Table 15 (p. 171).

Summary of socio-demographic differences. Socio-demographic differences were observed across all three primary outcome domains in the present study: (a) diabetesrelated characteristics, (b) BMI and physical activity characteristics, and (c) psychological characteristics. As such, these differences were taken into consideration in the testing of the present study's hypotheses.

\section{Hypothesis Testing}

\section{$\operatorname{Aim} 1$}

The first aim was to examine changes among key social-cognitive factors, as conceptualized in the motivational stage of the HAPA model, following presentation of recommended physical activity guidelines in DSME.

Hypothesis 1 (H1). It was hypothesized that there would be a statistically significant increase in participants' scores at Wave 2, when compared to Wave 1, on measures of (a) risk perception, (b) positive outcome expectancies, (c) task self-efficacy, and (d) behavioral intention, following presentation of DSME physical activity recommendations. This hypothesis was tested using a series of four paired samples $t$-tests to assess mean differences in participants' scores between Wave 2 and Wave 1. Each comparison was tested using an adjusted alpha of .0125 (Bonferroni correction $=.05 / 4=$ .0125). All four paired-samples $t$-tests were not statistically significant. 
Risk perception. The difference between Wave 2 risk perception $(M=7.98, S D=$ $2.76, n=150)$ and Wave 1 risk perception $(M=8.06, S D=2.52, n=150)$ was not statistically significant: $t=-.413, d f=149, p=.68$. The paired samples correlation between Wave 2 and Wave 1 risk perception was $r=.60, p<.001$.

Positive outcome expectancies. The difference between Wave 2 positive outcome expectancies $(M=71.94, S D=15.07, n=150)$ and Wave 1 positive outcome expectancies $(M=69.65, S D=13.83, n=150)$ was not statistically significant: $t=1.77$, $d f=149, p=.08$. The paired samples correlation between Wave 2 and Wave 1 positive outcome expectancies was $r=.40, p<.001$.

Task self-efficacy. The difference between Wave 2 task self-efficacy $(M=5.74$, $S D=2.57, n=150)$ and Wave 1 task self-efficacy $(M=5.66, S D=2.65, n=150)$ was not statistically significant: $t=.421, d f=149, p=.68$. The paired samples correlation between Wave 2 and Wave 1 task self-efficacy was $r=.61, p<.001$.

Behavioral intention. The difference between Wave 2 behavioral intention $(M=$ 4.18, $S D=1.50, n=150)$ and Wave 1 behavioral intention $(M=4.21, S D=1.36, n=$ 150) was not statistically significant: $t=-.413, d f=149, p=.68$. The paired samples correlation between Wave 2 and Wave 1 behavioral intention was $r=.60, p<.001$.

Hypothesis 1 supplemental analysis. Two additional paired-samples $t$-tests were conducted predicated on the possibility that participants in this study were operating within the volitional stage of the HAPA model prior to beginning DSME. It was hypothesized that if participants were already in the volitional stage at the beginning of DSME, scores on volitional measures at Wave 2 would be higher relative to scores at 
Wave 1 after participation in DSME. Each comparison was tested using an adjusted alpha of .025 (Bonferroni correction $=.05 / 2=.025)$.

Barrier self-efficacy. The difference between Wave 2 barrier self-efficacy $(M=$ $50.76, S D=22.36, n=152)$ and Wave 1 barrier self-efficacy $(M=52.10, S D=22.39, n=$ 152) was not statistically significant: $t=-1.01, d f=151, p=.31$. The paired samples correlation between Wave 2 and Wave 1 barrier self-efficacy was $r=.65, p<.001$.

Action planning. The difference between Wave 2 action planning $(M=2.77, S D=$ $.90, n=152)$ and Wave 1 action planning $(M=2.58, S D=.93, n=152)$ was statistically significant: $t=2.59, d f=151, p=.01$. The paired samples correlation between Wave 2 and Wave 1 action planning was $r=.51, p<.001$. Cohen's $d$ for correlated samples was calculated to determine the effect size of the difference between Wave 2 and Wave 1 action planning (Cohen et al., 2003; Dunlap et al., 1996). The effect size was considered small by Cohen's estimates: Cohen's $d=.21$.

\section{Aim 2}

The second aim was to address a series of questions examining the relationships and causal pathways among key social-cognitive factors, as theorized by the HAPA model, in the prediction of physical activity initiation following presentation of physical activity guidelines in DSME.

Question 1. Informed by the HAPA theory's conceptualization of a motivational stage, did risk perception, positive outcome expectancies, and task self-efficacy predict the formation of a behavioral intention to engage in physical activity?

Hypothesis 2 (H2). Following presentation of DSME physical activity guidelines, it was hypothesized that the formation of a behavioral intention to engage in physical 
activity at Wave 2 was predicted by the combination of Wave 2 measures (a) risk perception, (b) positive outcome expectancies, and (c) task self-efficacy, after controlling for potential covariates (see Figure 4). This hypothesis was tested using a hierarchical multiple linear regression model. The dependent variable (DV) was Wave 2 behavioral intention.

Correlation analysis. A correlation analysis was conducted to ensure proper model specification for the $\mathrm{H} 2$ regression analysis (Cohen et al., 2003; Garson, 2010b). Variables were selected for the correlation analysis a priori. These included the three HAPA motivational stage variables (risk perception, positive outcome expectancies, and task self-efficacy), and potential covariates selected based on (a) established empirical support and (b) relationships observed in the present study's analysis of differences among study variables as noted above (Marcus et al., 2000; Schwarzer, 2008). Correlations using Pearson's $r$ (for interval data) and Spearman's rho (for interval by nominal/ordinal data) were calculated. Relationships found statistically significant at the $p<.05$ level are listed in Table 16 (p. 172), and were entered into the regression equation as described below. Contrary to the HAPA model's conceptualization of a motivational stage, Wave 2 risk perception was unrelated to Wave 2 behavioral intention, $r=.07, p=$ .41. Wave 2 risk perception was subsequently omitted from the regression analysis (Garson, 2010b).

Regression analysis. A hierarchical linear multiple regression was calculated with the DV (Wave 2 behavioral intention) regressed onto two sets or blocks of predictors. Block 1 incorporated the control variables: (a) Wave 1 behavioral intention (a continuous score), (b) Wave 2 LTPA (a binary coded variable of 1: meeting or 0: not meeting LTPA 
recommendations), (c) depressive symptomology (CES-D 10 continuous score), and (d) annual household income (a dummy-coded variable of 1 : income $>\$ 40,000$ and 0 : income $<\$ 39,000$ ). Block 2 incorporated the independent variables: (a) Wave 2 positive outcome expectancies (a continuous score), and (b) Wave 2 task self-efficacy (a continuous score). Model 1 predicting Wave 2 behavioral intention from the Block 1 covariates was statistically significant: $F(5,133)=25.46, p<.001$ and accounted for $47 \%$ of the variance in Wave 2 behavioral intention: adjusted $R^{2}$ of .47 . Model 2 predicting Wave 2 behavioral intention from the Block 2 variables, while controlling for the effects of the Block 1 covariates, was also statistically significant: $F(7,131)=39.43$, $p<.001$ and accounted for $66.1 \%$ of the variance in Wave 2 behavioral intention: adjusted $R^{2}$ of .661. This was an $R^{2} \Delta$ of .189 over Model 1, indicating Model 2 was the superior model for predicting Wave 2 behavioral intention, accounting for $18.9 \%$ more variance in Wave 2 behavioral intention than Model 1.

Model 2 regression coefficients, standard errors, $\beta$ weights, and $95 \%$ Confidence Intervals are summarized in Table 17 (p. 173). As noted in Table 17, both Wave 2 task self-efficacy $(\beta=.561, p<.001)$ and Wave 2 positive outcome expectancies $(\beta=.113, p$ $<.05$ ), contributed to the prediction of Wave 2 behavioral intention, even after controlling for the effects of covariates. In addition to these independent predictors, it should be noted that two control variables, Wave 1 behavioral intention $(\beta=.185, p=$ $.005)$ and Wave 2 LTPA $(\beta=.122, p<.05)$ remained significant predictors of Wave 2 behavioral intention in Model 2. Model 2 is depicted in Figure 5 (p. 180).

Question 2. Informed by the HAPA theory's conceptualization of a volitional stage, did behavioral intention, barrier self-efficacy, and action planning predict 
engagement in physical activity after the presentation of DSME physical activity guidelines?

Hypothesis 3 (H3): It was hypothesized that the combination of Wave 2 HAPA variables (a) behavioral intention, (b) barrier self-efficacy, and (c) action planning would predict LTPA status (i.e., meeting or not meeting recommendations) at Wave 3, after controlling for potential covariates. This hypothesis was tested using a single hierarchical logistic regression model. The DV was defined as a binary of "1" meeting physical activity recommendations as taught in DSME and "0" not meeting physical activity recommendations as taught in DSME.

Correlation analysis. A correlation analysis was conducted to ensure proper model specification for the $\mathrm{H} 3$ regression analysis (Cohen et al., 2003; Garson, 2010b). Variables were selected for the correlation analysis a priori. These included the three HAPA volitional stage variables (behavioral intention, barrier self-efficacy, and action planning), and potential covariates selected based on (a) established empirical support and (b) relationships observed in the present study's analysis of differences among study variables as noted above (Marcus et al., 2000; Schwarzer, 2008). Correlations using Pearson's $r$ (for interval data) and Spearman's rho (for interval by nominal/ordinal data) were calculated. Relationships found statistically significant at the $p<.05$ level are listed in Table 18 (p. 174), and were entered into the regression equation as described below.

Regression analysis. A hierarchical logistic regression was calculated with the binary DV (1:0 of meeting Wave 3 LTPA recommendations) regressed onto two sets or blocks of predictors. The total $n$ for the logistical regression was 142, with 48 observed cases in the "1" group (i.e., meeting Wave 3 LTPA recommendations) and 94 observed 
cases in the "0" group (i.e., not meeting Wave 3 LTPA recommendations). Block 1 incorporated the control variables: (a) Wave 2 LTPA (as defined above), and (b) annual household income (as defined above). Block 2 incorporated the independent variables: (a) Wave 2 behavioral intention (a continuous score), (b) Wave 2 barrier self-efficacy (a continuous score), and (c) Wave 2 action planning (a continuous score).

In the absence of all predictors (covariates in Block 1 or independents in Block 2), it was possible to guess group membership correctly $66.2 \%$ of the time. In other words, if it was predicted that no one would meet Wave 3 LTPA recommendations (i.e., correctly determine classification of not meeting LTPA recommendations), one could correctly guess this purely by chance in the absence of any other information $66.2 \%$ of the time, which was statistically significant $(p<.001)$.

Model 1 predicting Wave 3 LTPA status from the Block 1 covariates was statistically significant $\chi^{2}=23.91, d f=2, p<.001$. Model 1 pseudo- $R^{2}$ estimates were .155 and .215 for Cox \& Snell $R^{2}$ and Nagelkerke $R^{2}$, respectively. Group membership for Model 1 could be predicted correctly $73.9 \%$ of the time, with better prediction of not meeting Wave 3 LTPA recommendations (88.3\%) compared to meeting recommendations (45.8\%), which was statistically significant for both covariates: Wave 2 LTPA recommendations $(p<.001)$ and annual household income $(p<.05)$. Model 2 (the full model) predicting Wave 3 LTPA status from the Block 2 variables, while controlling for the effects of Block 1 covariates, was also statistically significant $\chi^{2}=38.20, d f=5, p$ $<.001$. Model 2 pseudo- $R^{2}$ estimates were .236 and .327 for Cox \& Snell $R^{2}$ and Nagelkerke $R^{2}$, respectively. The Hosmer and Lemeshow goodness-of-fit chi-square test was not significant, $\chi^{2}=7.34, d f=8, p=.50$, indicating that the full model adequately fit 
the data (Garson, 2010a). Group membership for the full model could be predicted correctly $76.1 \%$ of the time, with better prediction of not meeting Wave 3 LTPA recommendations $(87.2 \%)$ compared to meeting recommendations $(54.2 \%)$.

The full model (Model 2) unstandardized logistic regression coefficients, Wald statistic, adjusted odds ratios, and $95 \%$ confidence intervals are summarized in Table 19 (p. 175). After controlling for Block 1 variables, only Wave 2 behavioral intention was statistically significant: Wald statistic $=6.13, d f=1, p=.01$; adjusted Odds Ratio $(\mathrm{OR})=$ $1.72,95 \% \mathrm{CI}=1.12$ to 2.64 . This suggested that Wave 2 behavioral intention increased the likelihood 1.72 times that a participant would meet Wave 3 LTPA recommendations. In other words, the odds of meeting Wave 3 LTPA are increased by a factor of 1.72 for every single unit increase of behavioral intention (Garson, 2010a). As noted in Table 19, no other HAPA model hypothesized predictors (e.g., Wave 2 barrier self-efficacy or action planning) were statistically significant. The full model (Model 2) is depicted in Figure 6 (p. 181).

Question 3. Did action planning mediate and/or a moderator the relationship between behavioral intention and physical activity engagement in the DSME population?

Hypothesis 4 (H4): It was hypothesized that action planning at Wave 2 would mediate, through simple mediation, the relationship between behavioral intention at Wave 2 and LTPA at Wave 3. This hypothesis was tested using two methods: (a) the causal steps process advocated by Baron and Kenny (1986), adapted to logistic regression (Herr, 2010; MacKinnon \& Dwyer, 1993), and (b) the Sobel $z$ test and Bootstrapping methods (Preacher et al., 2007; Preacher \& Hayes, 2004). All mediational analyses reported for $\mathrm{H} 4$ used a sample of 152 cases. 
Causal steps process. The causal steps process required that four criterion be met in order to establish action planning as either a partial or complete simple mediator of the behavioral intention - physical activity relationship. While the statistical process is slightly different between linear and logistic regression, the steps in general required estimation of four pathways: (a) path $c$ between predictor X (behavioral intention) and $\mathrm{Y}$ (the binary DV of physical activity); (b) path $a$ between the predictor X (behavioral intention) and the proposed mediator M (action planning); (c) path $b$ between the proposed mediator M (action planning) and Y (binary DV of physical activity); and (d) the path $c^{\prime}$ between predictor $\mathrm{X}$ on the $\mathrm{DV}(\mathrm{Y})$ is less (partial mediation) or zero (complete mediation) after controlling for the mediator, M (Baron \& Kenny, 1986; Herr, 2010; Preacher et al., 2007). Failure to meet any of these requirements (pathways $a, b, c$, and $c^{\prime}$ ) was tantamount to failure to finding a simple mediation effect of action planning.

Causal steps prediction of path $c$. Path $c$ between the predictor, Wave 2 behavioral intention and the DV, Wave 3 LTPA, was evaluated using a single logistical regression. The logistic regression model was statistically significant: $\chi^{2}=30.97, d f=1$, $p<.001$. The Wald statistic for behavioral intention $=22.62, d f=1, \mathrm{p}<.001$. This finding satisfied the first requirement of simple mediation.

Causal steps prediction of path $a$. Path $a$ between the predictor, Wave 2 behavioral intention and the hypothesized mediator, Wave 2 action planning, was evaluated with a single linear regression (as the DV, action planning, was continuous). It was statistically significant $F(1,150)=69.53, p<.001$. The unstandardized regression coefficient of Wave 2 behavioral intention predicting Wave 2 action planning was .336 ( $p$ $<.001)$. This satisfied the second requirement for simple mediation. 
Causal steps prediction of path $b$ and $c^{\prime}$. Path $b$ between the hypothesized mediator, Wave 2 action planning, and Wave 3 LTPA (binary DV of 1: meeting LTPA recommendations or 0 : not meeting LTPA recommendations) was evaluated with a single logistic regression $(N=152$ : group $1=49$, group $0=103)$ that yielded both path $b$ and the direct path $c^{\prime}$ (between the predictor Wave 2 behavioral intention and the DV Wave 3 LTPA, while controlling for the effects of the proposed mediator). While the overall model was statistically significant $\chi^{2}=33.19, d f=2, p<.001$, Wave 2 action planning (path $b$ ) was not: Wald statistic $=2.15, d f=1, p=.14$. Path $c^{\prime}$ was statistically significant: Wald statistic $=14.14, d f=1, p<.001$. Noting path $b$ was not supported (i.e., Wave 2 action planning, the proposed mediator, did not predict Wave 3 physical activity), this finding violated the third requirement of simple mediation (e.g., that the proposed mediator must affect the DV) (Baron \& Kenny, 1986; Preacher et al., 2007). As such, action planning did not mediate the relationship between behavioral intention and physical activity when evaluated by the causal steps process. Analyses then proceeded to the alternative statistical tests described below.

H4 alternative mediation analyses. As the causal steps process has been viewed as suffering from low power, additional analyses were conducted to evaluate action planning as a simple mediator between behavioral intention and physical activity. These tests included the Sobel $z$ test and Bootstrap methods (Preacher et al., 2007). Both statistical tests were conducted based on published recommendations for computing the Sobel $z$ and Bootstrap estimates, adapted to logistical regression (as the DV was the binary Wave 3 LTPA variable) (Herr, 2010; Preacher, 2009; Preacher \& Hayes, 2004). Consistent with the causal steps process, the Sobel $z$ test was statistically non-significant 
for the indirect path: $z=1.43, p=.15$. The obtained indirect value was .131 with a $95 \%$ $\mathrm{Cl}$ of -.05 to .31 . This finding suggested that action planning did not serve as a simple mediator of the behavioral intention - physical activity relationship.

Additionally, a Bootstrap method was used to evaluate action planning as a mediator (Preacher \& Hayes, 2004). In contrast to regression and the Sobel $z$ test, Bootstrapping does not yield a significance $p$-value but rather confidence intervals. Interpretation of the indirect path relies on whether the confidence interval spans across the number zero; if the confidence interval does not cross zero it is considered evidence that an indirect (i.e., mediator) effect was found (Preacher, 2010). The Bootstrap method used 5000 iterations to estimate the confidence intervals (an arbitrary but typical number of iterations used). These estimates yielded a mean of .13 and standard error of .09 . The $95 \%$ and $99 \%$ confidence intervals were: -.04 to $.34(95 \% \mathrm{CI})$ and -.09 to $.45(99 \% \mathrm{CI})$, respectively, both crossing the zero thresholds suggesting that the indirect effect of action planning was not found. Subsequently, all three statistical methods of $\mathrm{H} 4$ employed to test whether action planning served as a simple mediator of the behavioral intention physical activity relationship did not support the hypothesized mediator relationship.

Hypothesis 5. It was hypothesized that Wave 2 action planning would moderate the relationship between Wave 2 behavioral intention and Wave 3 LTPA (binary DV of 1: meeting LTPA recommendations or 0: not meeting LTPA recommendations). This hypothesis was tested using a hierarchical logistic regression $(N=152$ : group $1=49$, group $0=103$ ) with first-order predictors in Block 1 (Wave 2 behavioral intention and action planning), and the higher-order (interaction term) entered in Block 2. The interaction term of Wave 2 behavioral intention $\mathrm{x}$ Wave 2 action planning was created 
manually after centering scores. Centering was accomplished by subtracting the sample means from individual scores for Wave 2 behavioral intention and Wave 2 action planning prior to multiplying these factors together, producing the interaction term (Cohen et al., 2003; Garson, 2010a). The interaction term was correlated with both Wave 2 behavioral intention, Pearson's $r=-.30, p<.001$, and Wave 2 action planning, Pearson's $r=-.19, p=.02$. It was not correlated with the DV, Wave 3 LTPA, Spearman's rho $=-.06, p=.48$. While the overall logistic regression model was statistically significant: $\chi^{2}=33.60, d f=3, p<.001$, after controlling for first order effects of Wave 2 behavioral intention and Wave 2 action planning, the interaction term was not significant: Wald statistic $=.358, d f=1, p=.55$. 


\section{DISCUSSION}

\section{Summary of Results}

\section{Study Overview}

The present study sought to identify key social-cognitive determinants of physical activity initiation, as conceptualized by the Health Action Process Approach, among patients with type 2 diabetes attending Diabetes Self-Management Education. The HAPA model was selected as a theoretical framework due to its parsimonious structure and predominant focus on translating behavioral intention into actual behavior (Lippke \& Ziegelmann, 2008; Schwarzer et al., 2003; Schwarzer, 2008). Prior to the present study, no other studies had examined social-cognitive factors and physical activity, guided by health behavior theory, in the DSME population. Further, only two studies have been identified recently that examine social-cognitive predictors of physical activity, guided by health behavior theory, in the general type 2 diabetes adult population (Omondi, Walingo, Mbagaya, \& Othuon, 2010; Plotnikoff, Lippke, Courneya, Birkett, \& Sigal, 2010). For the remainder of this paper, the present study's sample population will be referred to as "DSME," as it best captures the participants in this study: patients with type 2 diabetes who were participating in DSME training.

The present study utilized a prospective, longitudinal, multi-site design in a midwestern metropolitan city to evaluate social-cognitive predictors and physical activity patterns of a moderate size $(N=152)$ convenience sample of patients with type 2 diabetes 
in DSME. Physical activity, diabetes-related variables, and SCFs of risk perception, positive outcome expectancies, task and barrier self-efficacy, behavioral intention, and action planning were measured using empirically supported self-report measures. Statistical procedures for this correlational study relied predominantly on regression analyses to test this study's hypotheses. Study measures were collected using selfadministered written and interviewer administered telephone-based surveys. Most participants in the present study were middle-aged adults and were women (73.2\%) who were Caucasian and had a post-high school education. Approximately half the sample was married. The median household income was approximately $\$ 39,999$ with most participants working full time or retired. As all outcome results used leisure-time physical activity, this will be referred to as "physical activity" from this point forward. Most participants engaged in a low-level of physical activity across all three study waves: $85.5 \%, 78.3 \%$, and $67.8 \%$, respectively, with the number of participants in low-level physical activity decreasing over time and transitioning into moderate-level physical activity.

The summary of results that follows is organized into two primary sections for ease of discussion. First, a summary and interpretation of primary findings is presented that reviews the results, interpretations, clinical implications, limitations, and future directions for each of the present study's hypotheses. Second, a summary of ancillary findings is presented to discuss results secondary to the primary aims of the study, such as socio-demographic differences, physical activity patterns, etc. These sections are then concluded with a review of general conclusions and study implications, strengths and limitations, and final comments. 


\section{Summary and Interpretation of Primary Findings}

\section{Aim 1}

The present study's first aim was to examine changes in social-cognitive factors, as conceptualized in the motivational stage of the HAPA model, following presentation of physical activity guidelines in DSME.

Hypothesis 1 (H1). This hypothesis posited that there would be a statistically significant increase in participants' scores at Wave 2, when compared to Wave 1 , on measures of HAPA motivational stage constructs: (a) risk perception, (b) positive outcome expectancies, (c) task self-efficacy, and (d) behavioral intention. Analyses revealed no statistically significant changes between Wave 2 and Wave 1 on any of the four constructs that comprise the HAPA motivational stage.

Due to this finding, supplementary analyses were conducted to examine HAPA volitional stage constructs of (a) barrier self-efficacy and (b) action planning. Supplementary analyses revealed a statistically significant difference for action planning but not barrier self-efficacy between Wave 2 and Wave 1. This finding indicated increased self-reported use of action plans at Wave 2, compared to Wave 1, and was considered a small effect by Cohen's definition of effect sizes (Cohen, 1992; Cohen et al., 2003). Support for these additional post-hoc analyses are discussed below. Interpretation of H1: This hypothesis was not supported in the present study. While it was difficult to determine specifically why there were no statistically significant changes in the HAPA motivational stage constructs, there are several possible explanations that could account for these findings. 
The first possible explanation highlights the fact that this was a non-experimental study. The social-cognitive factors were measured across multiple time points to evaluate the indirect impact of DSME's presentation of physical activity guidelines. Several studies have demonstrated the impact of experimental manipulations using these socialcognitive factors to promote health behavior change (Absetz et al., 2007; Laatikainen et al., 2007; Uutela et al., 2004). While these studies did not report data on changes in SCFs themselves (i.e., scores on SCFs for example were not reported, despite interventions specifically designed to affect them) it was inferred that changes in SCFs would then promote changes in the desired outcome behaviors that were reported (e.g., such as increases in physical activity, improvement in glycemic control, etc.). There is evidence in the literature that SCFs such as task self-efficacy are malleable, but the present study's non-experimental design was intended to only explore whether such changes in SCFs may be present following DSME. As such, it was not surprising that there were no significant changes detected as there was no direct experimental manipulation of conditions that could affect the HAPA motivational stage constructs. As noted throughout this paper, the literature does have a number of studies using correlational (i.e., nonexperimental) designs in which changes in social-cognitive factors are observed over time. This indicates that experimental manipulation (or lack thereof) by itself may not necessarily fully explain the current study's findings for $\mathrm{H} 1$.

A second possible explanation for these findings reflects uncertainty in the empirical literature regarding the temporal stability of social-cognitive factors. As many studies examining SCFs within the broader health behavior literature is correlational and cross-sectional in nature, many studies fail to report statistical analyses testing changes in 
SCFs over time. As such, very little is known as to how quickly or slowly SCFs may change when exposed to new information similar to what is presented in DSME. Of the existing literature on temporal stability of SCFs in the HAPA literature, most studies are limited to examining volitional stage constructs (Reuter et al., 2009; Sniehotta, Schwarzer, et al., 2005; Sniehotta et al., 2006; Ziegelmann et al., 2007). These studies indicated that behavioral intention and planning (action and coping) are relatively stable across periods as short as two months and up to three years. Unfortunately, these studies yield little information related to the temporal stability of the HAPA's motivational stage constructs tested in $\mathrm{H} 1$.

An additional study found that behavioral intention's prediction of behavior (as conceptualized by the Theory of Planned Behavior) was statistically moderated by intention's temporal stability (Conner, 2000). Conner's study provided additional support for the temporal stability of other constructs similar to SCFs conceptualized by the HAPA (e.g., attitudes similar to positive outcome expectancies), although it did not examine physical activity or a chronic illness population. While temporal stability may provide some insight as to the results found in $\mathrm{H} 1$, the lack of convergent or divergent empirical support from a comparable population using a similar temporal timeframe as the one in the present study makes it difficult to substantiate this explanation.

Finally, a third possible explanation for the $\mathrm{H} 1$ findings highlights the present study's design assumption that DSME participants were operating within the HAPA's motivational stage at baseline. In other words, given that the focus of the present study was on behavior initiation, and noting the lack of published findings on physical activity patterns in DSME, it was assumed that participants beginning DSME would be in a 
motivational stage of behavior change as conceptualized by the HAPA (Schwarzer, 2008). However, this may not have been the case. One potential reason for this could be that information presented in DSME that could affect risk perception, positive outcome expectancies, task self-efficacy, and behavioral intention may not have been necessarily novel to the participants. For many patients entering DSME, the importance of engaging in physical activity to manage obesity (a key predictor of type 2 diabetes), as well as the risks/complications associated with poorly managed diabetes, has previously been communicated by sources such as their healthcare providers, diabetes nurses, public health messages, etc. As such, when a patient attends DSME for the first time, s/he may have been primed and progressed past the HAPA's motivational stage.

It was not hypothesized a priori that DSME participants could have been operating within the HAPA's volitional stage at the start of DSME. While this was considered post-hoc, this realization led to supplementary analyses to examine the volitional stage HAPA constructs. As noted, a statistically significant difference was found for action planning, with participants reporting higher levels of action plans at Wave 2 compared to Wave 1 . While the reason for this change must be interpreted with caution due to the non-experimental design of the present study, it is possible that information learned specifically in DSME aided participants in formulating action plans. Standard DSME curriculum does promote action plans by teaching participants to address when, where, and how to effectively implement an intended behavior change (American Association of Diabetes Educators, 2009a; American Association of Diabetes Educators, 2009b; Diedrich et al., 2010; Mulcahy et al., 2003). 
Ultimately, it remains unclear what accounted for non-significant findings for all four motivational stage HAPA constructs. The three possible explanations provided are possible targets for future empirical exploration.

Clinical implications of H1: The lack of statistically significant findings for this hypothesis limits interpretation of the overall clinical implications for these results. Despite this, a supplementary analysis on volitional stage constructs, in particular action planning, suggested as one possibility that participants beginning DSME may actually be in a post-intentional, action-ready stage. If this were true, one prominent clinical implication for diabetes educators would be the opportunity to optimize the already limited classroom time to focus DSME curriculum on ways of enhancing behavior change. As the topic of physical activity is already heavily limited in DSME by the competing demands of other behaviors (e.g., blood glucose monitoring, diet), patients operating in a volitional, action-ready stage would facilitate a greater amount of time to discuss managing and overcoming barriers to activity and developing action and coping planning strategies. This is in contrast to spending time reviewing threat messages of why it is important to make such changes (Diedrich et al., 2010; Schwarzer, 2008).

Limitations of $\mathrm{HI}$. The findings reported for $\mathrm{H} 1$, limited strictly to differences observed in the action planning variable, must be interpreted with caution. A key limitation for the present study was that this was a non-experimental, correlational study design that inherently limits causal inferences. Any changes noted (e.g., the small effect of action planning) could certainly be due to factors other than an effect of DSME. Therefore $\mathrm{Hl}$ 's findings must consider that changes in action planning, or any other SCFs (had they been found) may have been the result of other potential influences. 
An additional potential limitation was the lack of well-established, psychometrically sound instrumentation. This was specifically true of measures of risk perception and positive outcome expectancies, as the psychometric properties for each of these measures were sub-par compared to other SCFs in the present study.

Future directions from $\mathrm{Hl}$ : As very little is known about the temporal trajectory of social-cognitive factors in DSME beyond the present study, future empirical studies should consider two possible alternatives. First, similar studies in the DSME should be conducted to better understand how SCFs, as formulated by the HAPA model, change over time. Second, clarification of which SCFs clearly play a role in modifying behavior will only come from more rigorous experimental designs. Study designs that incorporate both experimental manipulations as well as randomized, controlled settings, would help establish which social-cognitive factors are paramount in motivating and enacting behavior change. Such an approach would also further the process of removing redundancies and streamlining SCFs in health behavior theory promoted by Schwarzer's conceptualization of the HAPA (Schwarzer, 1992; Schwarzer et al., 2003; Schwarzer, 2008; Sniehotta , Scholz, et al., 2005). Experimental designs would further empirical understanding of both the HAPA and social-cognitive factors in general to promote healthy behavior.

\section{Aim 2}

The present study's second aim was to address a series of questions examining the relationships and causal pathways among social-cognitive factors theorized by the HAPA model to predict physical activity initiation. These questions are described and addressed below. 
Hypothesis 2 (H2). This hypothesis addressed the question of whether behavioral intention to engage in physical activity was predicted by the linear combination of (a) risk perception, (b) positive outcome expectancies, and (c) task self-efficacy, as proposed by the HAPA motivational stage. The present study sought to examine the relationship among these SCFs at Wave 2, after DSME presented guidelines on physical activity. Analyses revealed that behavioral intention was predicted by task self-efficacy and positive outcome expectancies, even after controlling for Wave 1 behavioral intention and physical activity. Risk perception was not associated with behavioral intention. Consistent with the HAPA model, task self-efficacy was the most prominent predictor of behavioral intention, followed by positive outcome expectancies. The study's findings explained $66.1 \%$ of the variance in Wave 2 behavioral intention for physical activity.

Interpretation of $\mathrm{H} 2$. $\mathrm{H} 2$ was partially supported in the present study. Contrary to a number of previous studies (Scholz et al., 2005; Schwarzer et al., 2008; Sniehotta, Scholz, et al., 2005), risk perception was not related to behavioral intention and subsequently was not entered into the regression equation. Lack of support for risk perception as a predictor of behavioral intention has only been reported in one other HAPA based study on physical activity (Schwarzer et al., 2007). However, risk perception is not unique to the HAPA literature. Some theorists have been led to question the role of risk perception in predicting behavioral intention or its utility to health behavior theories in general (Armitage \& Conner, 2000; Conner, 2008; Sutton, 2008).

With respect to positive outcome expectancies and task self-efficacy, the present findings were consistent with previously published studies (Scholz et al., 2005; Schwarzer et al., 2007; Schwarzer, 2008; Schwarzer et al., 2008; Sniehotta, Scholz, et al., 
2005). The present study supported task self-efficacy as the strongest predictor of behavioral intention, followed by positive outcome expectancies. The amount of variance in behavioral intention accounted for by this study's analyses was comparable to previously published percentages: $66.1 \%$ in the present study versus $65 \%$ to $69 \%$ in studies listed above.

While the present study's findings for $\mathrm{H} 2$ diverge slightly from previously published HAPA literature on motivational stage social-cognitive factors, it should be noted that the role of risk perception and positive outcome expectancies are conceptualized as relatively distal to both behavior and behavioral intention (Schwarzer et al., 2003; Schwarzer, 2008). The finding that risk perception was not related to behavioral intention yields interesting information unique to the DSME population but it may not necessarily be unique to the HAPA's formulation. As noted above, the role of risk perception is believed to be more important to novel health promoting behaviors typical of health screenings (e.g., breast or prostate cancer examination), yet may not necessarily be a key factor in behavior change in more complex and redundant behaviors such as physical activity (Schwarzer et al., 2003; Schwarzer, 2008).

While positive outcome expectancies were found to be statistically significant in $\mathrm{H} 2$, their contribution relative to task self-efficacy was small, consistent with comments by Schwarzer (2003) regarding the relative importance of SCFs in the HAPA model's motivational stage. In DSME, it is quite possible that positive outcome expectancies play a greater role in the development of task self-efficacy in contrast to behavioral intention, a suggestion consistent with previous writings on the HAPA model as well as socialcognitive theory in general (Bandura, 1997; Schwarzer, 1992; Schwarzer et al., 2003; 
Schwarzer, 2008). Consistent with the broader HAPA and social-cognitive literature, task self-efficacy in the present study proved to be the most important social-cognitive predictor of behavioral intention (Armitage \& Conner, 2000; Bandura, 1997; Conner, 2008; Schwarzer, 1992; Schwarzer, 2008).

Clinical implications of H2. The present study's findings suggest that in the DSME population, behavioral intention to engage in physical activity was predicted by the combination of positive outcome expectancies and task self-efficacy, even after controlling for the effects of baseline intention and physical activity. The findings yielded greater support for the role of task self-efficacy, consistent with both the HAPA and the broader health behavior theory literature. Noting the role of positive outcome expectancies and lack of findings in support of risk perception, clinical implications for the DSME setting suggest that emphasis should be placed on developing task selfefficacy. In practice, as noted below, this would allot a greater amount of time for diabetes educators to promote strategies that may be more likely to result in behavior change, rather than attempting to influence risk perceptions and outcome expectancies.

DSME by its very structure serves to develop task self-efficacy via verbal persuasion, one of the four sources of information that contributes to the development of self-efficacy (Bandura, 1997). Diabetes educators could potentially influence the other three sources of information reported by Bandura: performance accomplishments (i.e., engagement in physical activity), vicarious experience (i.e., watching others perform the behavior), and physiological feedback (from performing physical activity behaviors). By facilitating the development of self-efficacious beliefs via these additional pathways, the likelihood that patients in DSME would formulate behavioral intentions to engage in 
physical activity should, according to the HAPA model, also be increased. This process could be accomplished by encouraging patients at the beginning of DSME to engage in physical activity and monitoring this behavior through the use of a diary or pedometer / accelerometer devices, which have been demonstrated to improve physical activity participation in DSME settings (Diedrich et al., 2010).

While time constraints in DSME could serve as a barrier to implement this approach, one could equally argue that information provided in DSME that serves to highlight the risks and complications associated with poor glycemic control may be relatively unnecessary. This argument is predicated on the possibilities that patients (a) may enter DSME with already well-established risk perceptions associated with the costs of not engaging in health promoting behaviors, and (b) such information on risks/complications, based on the present study's findings, fail to contribute to the development of behavioral intentions needed to make the desired behavioral changes. In other words, abandoning DSME curriculum that promotes diabetes-specific health risk information may be an important move to accommodate promotion of task self-efficacy in DSME. Recent revisions to DSME standards are paving the way for making changes in the delivery of diabetes education (American Association of Diabetes Educators, 2009a; Funnell et al., 2010). While new approaches in general will likely improve the delivery of DSME, the lack of published research on social-cognitive factors within the type 2 diabetes and DSME populations indicates that much work remains to translate these results into practice.

Limitations of H2. Key limitations to $\mathrm{H} 2$ 's findings are related to design and measurement issues. As previously noted, the present study's non-experimental design 
limits causal inferences from the presented data. While the relationships revealed in $\mathrm{H} 2$ 's regression equation were strong, they remain correlational in nature. As such, one must interpret these findings with caution in order to preserve their importance to the empirical literature while at the same time not rigidly accepting them as dogmatic, rigorous evidence for the relationships that were discovered.

Additionally, conclusions drawn on both risk perception and positive outcome expectancies may in part be due to measurement issues. As noted above, there exists no gold standard in the empirical literature for measuring these (as well as most) socialcognitive constructs. The risk perception construct has proven repeatedly to be unwieldy, with inconsistencies in the empirical literature on (a) how to define risk perception, and (b) how to measure it. In the present study, the risk perception measure suffered from a large amount of missing or unusable data. This suggests that risk perception (and its underlying constructs of severity and vulnerability) have yet to be measured with an effective and stable instrument (Lewis, Jennings, Ward, \& Bradley, 1990; Lewis \& Bradley, 1994).

Similar difficulties exist with the measurement of positive outcome expectancies. The measure used in the present study yielded sub-optimal internal consistency and, like its other social-cognitive counterparts, an established, empirically supported instrument has yet to be found. Findings in the present study as they relate to positive outcome expectancies must be interpreted with caution due to the possible effect of measurement error, which can affect both the measured construct as well as the overall regression model tested (Cohen et al., 2003; Garson, 2010b). Clarification of the role of positive outcome expectancies (as well as the potential effects of negative outcome expectancies) 
will remain a challenge to health behavior theory research in the absence of well-defined and empirically tested measures (Williams et al., 2005).

Future directions from H2. Prior to the present study, little to no published research had examined key social-cognitive factors that may be important to the formation of a behavioral intention to engage in physical activity in the type 2 diabetes population. In fact, only two recent studies identified in the literature have taken on this task using the Theory of Planned Behavior (Omondi et al., 2010; Plotnikoff et al., 2010). Further, no studies using the HAPA model have been found that examined physical activity within DSME.

Future empirical explorations should consider extending the present study's $\mathrm{H} 2$ findings through the use of more powerful statistical methods, such as path analysis or structural equation modeling (Kline, 2005). While similar to regression, both path analysis and to a greater extent SEM would facilitate greater understanding of the relationships tested in $\mathrm{H} 2$. The relationships of risk perception and positive outcome expectancies with other HAPA motivational stage constructs should be further explored within the DSME population, as it remains unclear how these factors may relate to one another (Schwarzer et al., 2003; Schwarzer, 2008). The ability to concurrently assess these relationships via SEM would contribute to the greater understanding of which social-cognitive factors may be most important in physical activity initiation within the DSME setting.

Additionally, the lack of psychometrically sound instrumentation will limit future research endeavors with respect to the current findings. Future directions should incorporate development of empirically sound questionnaires to measure the social- 
cognitive factors believed to play a role in the prediction of behavior through the HAPA's motivational stage constructs.

Hypothesis 3 (H3). This hypothesis addressed the question of whether socialcognitive factors conceptualized by the HAPA's volitional stage predicted engagement in physical activity following presentation of DSME physical activity recommendations. Specifically, the present study sought to determine whether volitional stage constructs at Wave 2, (a) behavioral intention, (b) barrier self-efficacy, and (c) action planning, would predict which participants would meet the minimum recommended amount of physical activity at Wave 3, after controlling for the effects of covariates.

Analyses revealed several surprising results. The first finding was that in the absence of any additional information, one could guess correctly two-thirds of the time which participants, six to eight weeks after DSME guidelines were presented, would not meet the recommended amount of physical activity. The second finding indicated that inclusion of behavioral intention uniquely improved the overall prediction of whether a participant met minimum recommendations, after controlling for past physical activity behavior. The effect was marginal, noting that both the inclusion of behavioral intention and previous physical activity improved prediction of physical activity status by an additional $10 \%$. This effect suggested better (i.e., more correct) prediction of those participants not meeting recommendations compared to those meeting recommendations. Additionally, the present finding indicated that an increase or strengthening of behavioral intention improved the odds of meeting physical activity recommendations. The third finding revealed that while behavioral intention was found to improve this prediction, the effect for barrier self-efficacy and action planning was not statistically significant. 
Interpretation of $\mathrm{H} 3 \mathrm{H} 3$ was partially supported in the present study. The finding of behavioral intention as a key determinant of participation in physical activity was consistent with all of the known published literature on physical activity and the Health Action Process Approach, in addition to the broader health behavior theory literature (Armitage \& Conner, 2000; Conner, 2008; Lippke \& Ziegelmann, 2008; Schwarzer, 2008; Sutton, 2008; Weinstein et al., 1998). In contrast, much of the published HAPA literature on physical activity diverges from the present study's findings with respect to the role of both barrier self-efficacy and to a larger extent, action planning (Scholz et al., 2005; Schwarzer et al., 2007; Schwarzer et al., 2008; Sniehotta, Scholz, et al., 2005; Ziegelmann et al., 2007). In each of these studies, action planning contributed unique variance to the prediction of physical activity. Such findings have yielded discussions regarding the role of action planning as a mediator (discussed below in Hypothesis 4) and have traditionally been argued to address the intention-behavior gap (Lippke \& Ziegelmann, 2008; Schwarzer, 2008). Despite this, no studies have previously examined action planning in the type 2 diabetes or DSME populations.

One possible explanation for the present finding with respect to action planning is that participants in DSME may not have formulated idiosyncratic planning strategies to a level that might help translate intention into action. As the measurement of Wave 2 action plans was only several weeks after the start of DSME, this may have been too soon for the plans to be fully developed and implemented.

With respect to barrier self-efficacy, the present study's findings suggest that selfefficacious beliefs to overcome obstacles did not improve prediction of those participants who met physical activity recommendations. The reasons for this are likely similar to 
those discussed above with respect to action planning. Another possible explanation for failure to find a statistically significant effect for barrier self-efficacy may be related to the HAPA model's formulation itself. Barrier self-efficacy (also defined as coping selfefficacy) has often been studied in the empirical literature as a key determinant of maintained behaviors, such as regular physical activity (Schwarzer et al., 2003;

Schwarzer, 2008). Recent evidence shows that barrier self-efficacy may serve as a mediator between a direct effect of task self-efficacy and behavior itself (Scholz et al., 2005; Sniehotta, Scholz, et al., 2005), albeit the effect of barrier self-efficacy on behavior has not been consistent (Schwarzer et al., 2007). As such, the present study's findings may highlight the fact that barrier self-efficacy in the DSME population either (a) does not play a role in physical activity initiation, or (b) development of barrier self-efficacy takes longer than the timeframe in which it was measured (e.g., roughly four weeks). Neither of these possible explanations could be substantiated with the present study's methodology and data analysis, and therefore should be interpreted with caution.

Another possibility that may explain the current findings may have been related to the statistical methods employed by H3. As previously noted, the present study originally was designed to test this hypothesis using linear regression, consistent with the broader HAPA literature that typically employs linear regression, path analysis, and SEM. Due to the extremely non-normal distribution of physical activity scores, linear regression was abandoned in favor of logistic regression. As the present study was not originally powered for logistic regression, it is possible that failure to find statistically significant effects of action planning and/or barrier self-efficacy in $\mathrm{H} 3$ may have been related to an underpowered analysis. This possible explanation is not entirely conclusive, 
as empirical evidence suggests that the present study was sufficiently powered based on previous simulation studies of logistic regression (Vittinghoff \& McCulloch, 2007). This latter point is further substantiated by the fact that an effect for behavioral intention was found.

Finally, it remains quite possible that, of those social-cognitive factors measured, only behavioral intention serves to add to the prediction of who will meet or not meet physical activity recommendations six to eight weeks following presentation of DSME guidelines. Behavioral intention only slightly improved this prediction, after controlling for the effects of prior physical activity. When combined with prior physical activity, the prediction of physical activity at Wave 3 was still statistically significant, yet this combination only marginally improved prediction of physical activity compared to simply guessing. This explanation highlights the finding that most of the DSME sample in the present study predominantly failed to meet the minimum physical activity recommendations across all three measurements. Considering that one could guess a patient's physical activity status correctly two-thirds of the time strictly by chance and without any other information, the present study's H3 findings dramatically highlight the need to re-evaluate DSME curriculum and methods to promote physical activity initiation.

Clinical implications of H3. These findings have two key implications for clinical practice in the DSME setting in particular, as well as in the type 2 diabetes population in general. First, these findings provide additional evidence in support of understanding and influencing a patient's behavioral intention to engage in physical activity. As intention increases, so too does the likelihood that a patient will initiate behavior and engage in the 
minimum levels of recommended physical activity within weeks of participating in DSME. As behavioral intention is theorized to be influenced by task self-efficacy by the HAPA model and as findings in the present study indicated, diabetes educators should focus their efforts on promoting the development of self-efficacy for engaging in physical activity, as discussed above. This remains a lofty goal and challenge, since it has been previously been noted that physical activity is often relegated to a second-class status in DSME in favor of self-management behaviors that may be more amenable to the provision of information only, such as nutritional guidance (Diedrich et al., 2010; Funnell et al., 2010). While this is often due to time and financial cost constraints, initial support from the present findings and empirical support from pre-diabetes populations highlight the need to expand DSME to focus on SCFs that could influence engagement in physical activity (Absetz et al., 2007; Laatikainen et al., 2007; Uutela et al., 2004).

Second, the unexpected finding of $\mathrm{H} 3$ highlights that a diabetes educator or other healthcare provider, meeting with a patient six to eight weeks following DSME instructions, may simply guess whether a patient is meeting the minimum physical activity recommendations and be accurate two-thirds of the time. The clinical implications for this finding are tantamount to difficulties assessed in DSME almost a decade ago (Norris et al., 2001). Considerable work remains in addressing physical activity initiation and adoption in the DSME population. These findings also underscore the overall difficulty in initiating a behavior such as physical activity, especially in the context of other competing self-management behaviors. As such, these findings suggest that an interdisciplinary approach to behavior change is necessary, and highlights that 
providing purely didactic information alone may be insufficient to translate behavioral intention into action.

Limitations of H3. Perhaps the single most prominent limitation to $\mathrm{H} 3$ was related to the methodological and statistical difficulties that presented post-hoc. Due to the extreme non-normal distribution of physical activity scores, logistic regression was performed in lieu of linear regression, as described above. While an argument has been made that this test was sufficiently powered, this remains a limitation noting the study was not originally designed to use logistic regression (Babyak, 2004; Vittinghoff \& McCulloch, 2007). As such, these findings must be interpreted with caution due to H3's analysis being (a) post-hoc by design, and (b) correlational in nature.

An additional limitation of $\mathrm{H} 3$ findings reflects divergence from previous HAPA literature in that it is difficult to directly assess the amount of variance accounted for in physical activity through the use of logistic regression. This limitation, while unique to this study, highlights greater concerns regarding the broader HAPA literature that will be discussed below in the general limitations section.

Future directions from $\mathrm{H} 3$. The use of a categorical dependent variable in health behavior literature is neither new nor unique, but its use in the HAPA literature, and the broader health behavior theory literature, appears limited in favor of continuous outcome variables. The use of categorical cut-offs, such as meeting or not-meeting physical activity recommendations, yields the potential for novel methodological approaches that are not limited by current statistical procedures, as many of the more advanced multivariate statistics have been adapted to logistic regression and other tests that 
represent extensions of the general linear model (e.g., generalized estimating equations) that may use dichotomous or multi-level outcome variables (Garson, 2009).

Clearly a future direction must be to clarify the role of action planning within the DSME population. Initial research into the functional role of action planning was addressed by $\mathrm{H} 4$, discussed below. With respect to barrier self-efficacy, its theoretical function in behavior initiation is less clear. While it has been conceptualized as predicting behavior initiation, empirical evidence suggests that this construct is a better predictor of maintained behavior (Schwarzer, 2008). As such, future research will be needed to determine whether barrier self-efficacy does predict physical activity initiation in the DSME setting, or if it should remain a construct specific to the maintenance of behavior.

Hypothesis 4 (H4). This hypothesis addressed the question of whether action planning mediated the intention - behavior relationship in the present study's sample. It was hypothesized that action planning would serve as a simple mediator (i.e., mediate the relationship) between behavioral intention and physical activity participation, which was consistent with the HAPA model's formulation. Three different statistical approaches were used to assess $\mathrm{H} 4$, yet all three failed to find the hypothesized mediator relationship. Interpretation of H4: This hypothesis was not supported by the present study's findings. The common factor across all three statistical approaches was noted above in $\mathrm{H} 3$, namely, that there was no direct effect of action planning on physical activity participation in the present study. This finding was confirmed by the third step of Baron and Kenny's (1986) causal steps process and supported by the additional tests using the Sobel $z$-test and Bootstrapping methods. 
Only one study among the HAPA / physical activity literature was found that concurred with the present study's finding (Schwarzer et al., 2007). In that sample of 365 internet surveyed participants in Germany, the indirect effect between behavioral intention and physical activity (i.e., the test of action planning as a simple mediator) was not supported. However, failure to find the mediating role of action planning has been observed in different populations looking at health behaviors other than physical activity (Conner, 2008; Schwarzer et al., 2007; Sutton, 2008) In contrast, most HAPA studies that have examined this relationship in the prediction of physical activity have discovered the simple mediator role of action planning (Lippke et al., 2009; Scholz et al., 2008;

Schwarzer et al., 2008; Sniehotta, Scholz, et al., 2005; Sniehotta, Schwarzer, et al., 2005; Wiedemann et al., 2009). There are three possible reasons that might explain the failure to discover the mediating role of action planning in the present study.

The first possible explanation, consistent with $\mathrm{H} 3$, considers whether the statistical analysis had sufficient power. The change from linear regression to logistic regression to address statistical assumptions introduced the possibility that $\mathrm{H} 4$ would also suffer a loss of power. While certainly a possibility, this is unlikely for several reasons. First, the causal steps process advocated by Baron and Kenny (1986) is believed to suffer from low power itself, a reflection on the statistical approach rather than the present study's design (Preacher et al., 2007; Preacher \& Hayes, 2004). Moreover, despite concerns regarding the causal steps process, it has been adapted to logistic regression and based on the present study's sample size and number of tested variables would likely have had sufficient power to test, at a minimum, the direct path between action planning and physical activity (Herr, 2010). The direct path, as noted above, was statistically not 
significant. Second, while the Sobel $z$-test could be affected by insufficient sample size, the Bootstrapping method is not as susceptible to this as the basic premise of this method is to randomly sample the available data and construct confidence intervals based on this random sampling. One of the HAPA studies reported (Wiedemann et al., 2009) was able to identify a simple mediator effect in post-hoc analyses using Bootstrapping for a sample of 124 participants (the present study's sample was 152). As such, it appeared the present study had adequate sample size and power to detect if action planning was to serve as a simple mediator.

The second possible explanation reflects a shift in recent HAPA research that has attempted to address the inconsistent findings of action planning serving as a simple mediator. Researchers have begun examining whether the mediating role of action planning is more complex than originally posited by the HAPA's simple mediation formulation. Several studies of the HAPA model and physical activity have examined moderated mediation, attempting to identify factors that might moderate or interact with the mediating relationship (Lippke et al., 2009; Scholz et al., 2008; Wiedemann et al., 2009). Their findings indicated that the mediating role of action planning may be moderated by (a) levels of behavioral intention, and (b) levels of self-efficacy. While these new findings provide additional information to the elaboration of the HAPA model and addressing the broader issue of the intention-behavior gap, significant challenges exist in identifying such complex relationships in the "real world." Each of these studies reported large to very large samples sizes, with the exception of Wiedemann et al., in which Bootstrapping methods were used to test moderated mediation. Unfortunately, the 
use of Bootstrapping methods in smaller samples, while informative, lack scientific rigor when used purely as a post-hoc analysis.

A third, final reason may be that action planning simply does not serve a direct or indirect (i.e., mediation) role in predicting physical activity initiation in the DSME population sampled in the present study. While certainly a possibility, this reason cannot be substantiated as it is tantamount to accepting the null hypothesis.

Clinical implications of $\mathrm{H} 4$ : With the present finding, there are no current clinical implications with respect to action planning's role in predicting physical activity initiation. Additional research is needed, as discussed below.

Limitations of H4: As the present hypothesis was not supported, there are no particular limitations to this finding.

Future directions from H4: Future research within the DSME and type 2 diabetes populations should focus on two key points based on the findings of H4. First, future studies will need to replicate these findings to determine whether action planning does directly influence physical activity behavior in this population. Second, clarification of how action planning affects physical activity initiation will be important to examine. This latter issue will require a priori study designs that account for both simple mediation, as well as moderated mediation, due to limited evidence that this relationship may be more complex than the HAPA model's current formulation.

Hypothesis 5 (H5). This hypothesis addressed the question of whether action planning moderated the intention - behavior relationship in the present study's sample. It was hypothesized that action planning (as an interaction with behavioral intention) would 
moderate the relationship between behavioral intention and physical activity. Analyses failed to find this relationship.

Interpretation of H5: This hypothesis was not supported by the present study's findings. While the role of statistical moderators have been explored with the HAPA literature, more recently in attempts to better explain the mediating role of action planning as noted above, no study identified has attempted to test whether action planning moderated the intention - behavior relationship. The suggestion to assess this potential relationship was proposed by theorists critical of the HAPA literature's limited empirical support and conceptualization of action planning as a key factor in closing the intention-behavior gap (Sutton, 2008). While it was possible that action planning may moderate this relationship, there is currently no evidence or empirical studies to support this.

With respect to the present study's $\mathrm{H} 5$ finding, failure to find this moderator relationship does not confirm that such a relationship does not exist in the DSME population. One plausible reason for failure to find an interaction effect comes from the fact that interaction effects in the real world setting are difficult to detect (McClelland \& Judd, 1993). It was quite possible that the present study simply lacked sufficient power or sample size to detect this difference. While increasing sample size would improve the likelihood of finding an interaction effect (assuming one exists), the feasibility of conducting a study with a large enough sample size presents serious challenges to researchers. 
Clinical implications of H5: With the present finding, there are no current clinical implications with respect to action planning as a moderator of the behavioral intention physical activity relationship in this study's DSME sample.

Limitations of H5: As the present hypothesis was not supported, there are no particular limitations to this finding.

Future directions from H5: While the question remains in both the present study and other published literature regarding whether action planning may moderate the intention-behavior relationship, there currently exists no identified literature that has tested this relationship. Moreover, recently published literature suggests that HAPA researchers are focused on better defining the mediational role of action planning (i.e., through moderated mediation) rather than action planning as a moderator between intention and behavior. This makes intuitive sense noting that the HAPA model does not view behavioral intention as the most proximal predictor of behavior, in contrast to other health behavior theories, such as the Theory of Planned Behavior. As such, future directions using the HAPA formulation should likely not include pursuing action planning as a moderator in the absence of large, population based data sets.

\section{Summary \& Interpretation of Ancillary Findings}

In addition to the present study's primary findings, a number of interesting findings ancillary to this study's purpose were found. These additional findings may further contribute to the empirical literature pertaining to social-cognitive factors and physical activity initiation in the type 2 diabetes and the DSME population. A summary of these findings is presented below. 
Socio-demographic differences in task self-efficacy. In the present study, gender differences were observed in task self-efficacy. Analyses revealed that women tended to have lower task self-efficacy than men at both time points measured. This finding must be interpreted with caution, noting that this study's sample was predominantly female. As such, the small number of males in the study may not necessarily represent task selfefficacy scores in males within the broader DSME population. Nevertheless, this finding has empirical support from the broader self-efficacy literature. Two studies were identified that lend support to the possibility that women beginning engagement in physical activity may report lower levels of initial task and barrier self-efficacy, but demonstrate an accelerated increase in self-reported self-efficacy in addition to physical activity behavior within a short amount of time (Blanchard, Rodgers, et al., 2002; Hankonen, Absetz, Ghisletta, Renner, \& Uutela, 2010). Blanchard et al.'s study revealed women reported less baseline task and barrier self-efficacy when beginning cardiac rehabilitation but quickly accelerated both their physical activity and self-efficacy scores. Hankonen et al.'s finding in a type 2 diabetes population found no baseline differences between male and female scores of self-efficacy, but women quickly demonstrated increased self-efficacy and associated physical activity behavior, outpacing men in the study. These findings offer insight into the fact that DSME interventions designed to enhance self-efficacy should not utilize a blanket approach. In other words, DSME interventions to increase self-efficacy should use a dose-response approach tailored differently between men and women. An example of this may be that interventions to improve self-efficacy may need longer duration or further instruction for male DSME participants compared to females. 
Gender differences highlighting differential effects of self-efficacy in women is an important finding, in particular when applied to the DSME population, as recent evidence tends to suggest that participation in DSME may be a female phenomenon (Gucciardi, Wang, DeMelo, Amaral, \& Stewart, 2008). The present study's findings would lend support to this idea leading DSME policy makers to question current strategies to involve men, since males are just as likely to develop type 2 diabetes (American Association of Diabetes Educators, 2009a).

The role of income. Previous research has demonstrated a number of sociodemographic barriers to engagement in physical activity initiation, including socioeconomic status (Estabrooks, Lee, \& Gyuresik, 2003; Marcus et al., 2000). In the present study, the most prominent socio-demographic factor that was related to both physical activity and most of the measured social-cognitive factors was annual household income, a measure of socio-economic status (SES). Annual household income for the present study yielded a median of approximately $\$ 39,000$, which was comparable to census data and DSME literature (Gucciardi et al., 2008). It remains unclear why annual household income demonstrated such a strong relationship with the primary study variables.

One possible explanation highlights differential effects of SES on physical activity patterns. Estabrooks et al. (2003) noted that among low and medium SES neighborhoods, there were less available free facilities accessible to the public, while still possessing as many pay-for-use facilities compared to upper SES neighborhoods. Reduced access to facilities is believed to reflect SES, which in turn is a reflection of annual household income. 
A second possible explanation is related to the unique financial circumstances present during the course of this study's recruitment period. Recruitment for this study occurred during the global economic crisis that began in the fourth quarter of 2008 , leading the U.S. and global economies into a recession that persists today (International Monetary Fund, 2009). While data has yet to be published on how the global economic crisis has affected diabetes education in general, or the U.S. specifically, the International Diabetes Federation recently published notes from their United Nations summit that highlighted increasing challenges to combating diabetes, partly attributed to the global economic crisis (International Diabetes Federation, 2010) . Further, as early as April, 2009 , media reports began noting the affects of the global recession on diabetes selfmanagement, with many people having to make difficult decisions about their care, such as skipping medical appointments or not refilling diabetes medication due to financial costs (The Associated Press, 2009). It was quite possible that the effects of the global economic crisis influenced the self-care behaviors, including physical activity, of study participants attending DSME.

These two explanations present interesting perspectives; however, the lack of empirical support highlights the uncertainty why annual household income was strongly related to the primary outcome measures of the present study. While this variable was associated with physical activity and social-cognitive factors, it failed to hold up when entered into the regression equations as a covariate. This may highlight another possibility: that annual household income covaried with another factor in the study, or perhaps an unmeasured variable that could explain these findings. 
As an aside, while it remained difficult to initially recruit participants into this study across the study's duration, retention between all three Waves was very good. This may in part have been due to the guaranteed $\$ 30$ gift card compensation and the possibility of winning a $\$ 100$ gift card in the lottery drawings. This may have explained differences between completers and non-completers, as most individuals in the lowest income bracket remained in the study, while most participants who left the study prematurely were in the middle income bracket. This may inform future longitudinal research in DSME settings that the use of gift card compensation may indeed assist retention, but more so for lower income participants.

BMI. Participants in the present study reported considerably higher BMIs compared to other DSME populations. With respect to this study's overall sample, most participants $(95.8 \%)$ had a BMI indicative of overweight or obese, with $30 \%$ in the highest World Health Organization obesity class (Class III) (World Health Organization, 2010). Relative to a comparable sample from two large DSME programs in Toronto, Canada, the mean BMI in the present study is higher than the Canadian sample by four points (Gucciardi et al., 2008). While African-American representation in the present study was comparable to local census data, the majority of African American participants was female and had a mean BMI higher than the overall study sample. Of the 20 AfricanAmerican women in the present study, most $(95.6 \%)$ had BMIs of overweight or greater, which is higher than observed figures in a large population based study of approximately 50,000 African-American women that reported $61 \%$ had BMIs of overweight or greater (Krishnan, Rosenberg, Djousse, Cupples, \& Palmer, 2007). The BMI results for the present study highlight the need in DSME settings to implement effective dietary and 
physical activity strategies to reduce BMI and associated health risks. The primary findings of the present study, in conjunction with these BMI results, reiterate the urgency and challenge to diabetes educators and other healthcare providers to encourage physical activity initiation in diabetes patients.

Physical activity patterns. The present study's physical activity measurements across three study Waves yielded key descriptive information missing in the DSME empirical literature. Across all three Waves most participants did not meet the minimum recommended amount of physical activity. Despite this, a trend was evident suggesting that participants as a whole were increasing their activity between Waves 1 and 3 , including some participants who achieved a high level of physical activity. These findings suggest that information presented in DSME was likely having an impact on physical activity initiation.

A unique finding was discovered related to the Wave 1 physical activity levels. As noted, $14.5 \%(n=22)$ of the sample engaged in a moderate level of physical activity at Wave 1 . This finding calls into question whether these participants self-selected into the study by failing to endorse one of the study's exclusion criteria that asks participants if they are currently engaging in activity for 30 accumulated minutes a day, four to five days a week. It is important to highlight that the definition of "moderate," was determined by the categorical classifications advocated by the IPAQ development committee (see Table 7) (Sjostrom et al., 2005). Part of that moderate level classification does incorporate the current $\mathrm{CDC} / \mathrm{ACSM}$ recommendation of 150-minutes per week. It also includes individuals who engage in three or more days of vigorous-intensity activity at least 20-minutes per day. A review of the data indicated three participants fit the 
moderate classification due to their vigorous-intensity activity, and as such would not have endorsed the exclusion item on the study's self-screener.

This leaves the remaining 19 participants who had moderately active IPAQ scores at Wave 1. It remains unclear why these individuals endorsed "no" on the screening item, thereby entering the study, but reported Wave 1 activity levels that would qualify for a moderate level of activity. While this calls into question whether these participants should have remained in the study, two key points must be stated. The first is the original intent of the screening measure was to prevent individuals who were exceeding the minimum physical activity recommendations from entering the study. As there were no identified published data on rates of physical activity participation in DSME prior to the present study, it was unclear how many participants would self-select out of the study. It should be noted that none of the participants at Wave 1 exceeded the minimum recommended amounts of physical activity (i.e., no one was in the "high" category). Second, with respect to the present study's purpose and hypothesis testing, the Wave 1 physical activity measurement was collected simply as a potential covariate to be controlled if it had demonstrated statistically significant relationships with other outcome measures of importance. In other words, the effect of Wave 1 physical activity was not an independent predictor of interest, and as such these few participants who were engaged in a moderate level of activity at baseline did not affect the results of the primary analyses in the present study. As such, these participants were kept in the study.

Overall, the present study revealed that far too many patients attending DSME are physically inactive or engaging in activity that just barely meets the minimum recommendations. Further discussion on this is presented below. 
Depressive Symptomology. Depressive symptomology in the present study was measured by the CES-D 10 and indicated that approximately half of the study's participants had a "positive flag" for probable depressive symptoms using a cut-off of $\geq$ 10 (Andresen et al., 1994; Center for Research on Women with Disabilities, 2009). If a cut-off score of 8 had been used, an even larger proportion of this study's sample would have been flagged "positive" for probable depressive symptoms. The present study's findings highlight two primary points: (a) methodological concerns about the utility of the CES-D 10, and (b) implications for clinical practice regarding potential rates of depression in the DSME setting.

Methodological concerns. First, as there was no published literature using the CES-D 10 in the DSME population, and only two studies found using the measure in diabetes populations in general, the present study's findings highlight methodological concerns about the CES-D 10 (Carnethon et al., 2007; Krein et al., 2005). In particular, it is possible that the CES-D 10 may actually reflect negative affect rather than depressive symptoms. The potential for this possibility comes from the fact that most patients attending DSME have been recently diagnosed with type 2 diabetes. As they enter DSME, these patients are confronted with an overwhelming amount of information that communicates the need to change their lifestyle behaviors and that these changes must be permanent. Evidence in support of this possibility comes from a population-based study of patients with type 2 diabetes $(n=506)$ using the larger, full scale CES-D (20-item version) (Fisher et al., 2007). Their study used structured interviews to validate the presence of major depression among those participants who scored in the depressive range of symptoms on the CES-D. Results revealed greater than $70 \%$ of those individuals 
who met criteria for depression using the CES-D were not clinically depressed.

Additionally, they discovered that despite the CES-D's over-reporting of depressive symptomology, there was very little functional difference with respect to diabetes selfmanagement between those who were clinically depressed and those who were not. Fisher et al. (2007) suggested two unique findings. First, that the CES-D may likely be a more appropriate measure of emotional (affective) distress in patients with diabetes, rather than a measure of depressive symptomology. Second, there were many functional similarities among those diagnosed with depression and those without. These similarities re-iterated that whether depression or simply affective distress, these symptoms were detrimental to effective diabetes self-management.

A second methodological concern is related to the use of different cut-off scores for the CES-D 10 in the empirical literature. In the diabetes literature, Krein et al. (2005) reported using a cut-off of 10, Carnethon et al. (2007) reported using a cut-off of 8 ; in the general literature cut-off scores as low as 4 have been reported (Irwin, Artin, \& Oxman, 1999). This variability in the limited empirical literature highlights the need to determine an appropriate CES-D 10 cut-off score that would represent both an empirically and clinically meaningful finding that could benefit patients and providers in DSME.

Future studies should consider measuring depressive symptomology in DSME with both the CES-D 10 and a measure of affect (e.g., the Positive and Negative Affect Scale - PANAS) to address the possible overlap of depressive symptomology with negative distress or affect. It would also be important to examine similarities and differences between the 10-item and 20-item CES-D within the DSME setting. Further, studies are also needed to examine differences between cut-offs for depressive 
symptomology on the CES-D 10 (e.g., $\geq 8$ versus $\geq 10$ ). Such information is needed to identify whether there is any empirical and/or clinical difference between a cut-off of two points and to better differentiate those patients who are depressed or at-risk for depression .

A review of the literature yielded no published data on the CES-D 10 in the DSME population and only one study was found that provided insight into the rates of possible depressive symptoms in the DSME setting (Gucciardi et al., 2008). Gucciardi et al.'s study, using the Beck Depression Inventory - II, revealed approximately $10.4 \%$ and $13.1 \%$ of its total sample $(n=275)$ endorsed mild and moderate symptoms of depressed mood, respectively. Moreover, the study revealed gender differences in self-reported depressive symptoms. Women $(n=143)$ tended to report mild and moderate symptoms of depression compared to men $(n=132)$, who endorsed more minimal symptoms. These findings highlight the need to further understand both the rates of depressive symptoms in DSME, and how this may present differently based on gender.

Clinical implications. Despite methodological concerns regarding the CES-D 10, the present study's findings highlight the need for diabetes educators to be alert to possible depressive symptomology among DSME participants. Fisher et al. (2007) indicated that there was very little functional difference with respect to diabetes selfmanagement between those who screened positive for depression, but were not clinically depressed, and those who were diagnosed with major depression. In either case, effective diabetes self-management was threatened, emphasizing the need to address potential depressive symptoms early in DSME. Additionally, whether emotional distress or depressive symptomology, empirical evidence suggests that stressful life events (such as 
being diagnosed with diabetes) can influence the development of depressive symptoms, which would then influence effective diabetes self-care (Maciejewski, Prigerson, \& Mazure, 2000). These findings highlight the need for future collaboration between DSME providers and mental health professionals in order to maximize translation of diabetes education curriculum into successful and enduring behavior change.

To date, no studies were identified examining the HAPA model and physical activity in conjunction with depressive symptomology. Evidence from the broader socialcognitive literature suggests that SCFs, in particular self-efficacy, can serve as a protective factor against depressive symptoms, but is also vulnerable to the effects of depression in the management of chronic medical illness (Jerant, Kravitz, Moore-Hill, \& Franks, 2008). Further research is needed to determine how depressive symptoms may interact with SCFs, and how such relationships may impact health behavior change in the DSME setting.

\section{General Conclusions and Implications}

The present study yielded three predominant contributions to the empirical literature. These included (a) insight into physical activity patterns of type 2 diabetes patients attending DSME, (b) clarification of which social-cognitive factors may predict physical activity initiation in this population, and (c) expansion of the current, yet limited HAPA literature as it applies to the diabetes population. A review of each of these domains will summarize the implications of these findings as applied to further empirical exploration and highlight considerations for future clinical practice. 


\section{Physical Activity and DSME}

Regular physical activity remains a key component of successful diabetes selfmanagement with significant empirical evidence supporting its role in delaying diabetesrelated complications (Albright et al., 2000; American Association of Diabetes Educators, 2009a; American Diabetes Association, 2002; American Diabetes Association, 2010; Zinman, Ruderman, Campaigne, Devlin, \& Schneider, 2002). People with type 2 diabetes attending DSME are in a key position to learn the recommended levels of physical activity and strategies to implement these recommendations. While the benefits of DSME to other self-management behaviors (e.g., diet, self-monitoring of blood glucose, foot care, etc.) has been well-established, limited evidence exists about DSME's effectiveness to significantly increase or improve physical activity behavior (American Association of Diabetes Educators, 2009a; Boren, Fitzner, Panhalkar, \& Specker, 2009; Davies et al., 2008; Norris et al., 2001). Findings in the present study revealed that most participants during and several weeks following DSME failed to demonstrate changes in their physical activity patterns.

As the present study was focused on identifying social-cognitive predictors of physical activity initiation in DSME, rather than evaluating the effectiveness of DSME, inferences drawn from this study regarding the effectiveness of DSME must be cautioned. However, one observation from a review of the literature suggests that there may be a clinical disconnect between how much physical activity is recommended in the empirical literature and what recommendations and strategies are presented within the limited timeframe of DSME. Recent revisions to the physical activity literature, based on reviews of randomized trials, indicate that current recommended amounts of physical 
activity (e.g., 150 minutes of moderate-intensity activity per week) represent the barest minimum of activity to prevent weight gain and manage chronic disease complications (Donnelly et al., 2009). However, these recommendations from the ACSM recognize, "there is likely a dose effect of PA [physical activity], with greater weight loss and enhanced prevention of weight regained with doses of PA that approximate 250 to 300 [minutes a week] of moderate-intensity PA (Donnelly et al., 2009, p. 467).

Recognizing the difficulty of patients to meet even the minimum recommendations, educational materials often promote accumulated levels of activity, in minimum bouts of 10 -minutes, with suggestions including parking further from work, using stairs, etc. (American Association of Diabetes Educators, 2009a; American Association of Diabetes Educators, 2009b). These recommendations recognize that initiation of behavior change requires small, manageable, and achievable steps. However, it remains unclear whether these recommendations may be sufficient to help patients in DSME achieve the minimum recommended levels of physical activity. Donnelly et al.'s (2009) findings highlight a problem that surpasses the DSME population and may likely reflect to a greater degree general difficulty facing public health: translating empirical evidence into clinical application.

An important clinical implication for diabetes educators and DSME curriculum in general may be the need to re-evaluate how physical activity recommendations are promoted in the DSME setting. This would entail (a) explicitly discussing how much physical activity really is needed to produce benefits to diabetes outcomes (i.e., presenting the actual, evidence-based recommendations necessary to lose weight and manage diabetes symptoms), and (b) developing and implementing strategies that would 
affect the mechanisms involved in physical activity behavior change. This latter requirement, discussed below, reflected the predominant focus of the present study.

\section{Social-Cognitive Factors in DSME Physical Activity Initiation}

Findings in the present study offer a preliminary understanding of the socialcognitive factors that may affect physical activity initiation in people with type 2 diabetes who participate in DSME. These SCFs include the predominant constructs conceptualized in the broader health behavior theory literature and have considerable empirical support across a myriad of health behaviors and chronic illness populations (Armitage \& Conner, 2000; Conner, 2008; Lippke \& Ziegelmann, 2008; Renner \& Schwarzer, 2003; Schwarzer, 1992; Schwarzer, 2008; Sutton, 2008). As the current literature on SCFs and physical activity initiation within the DSME population is extremely limited, the present study's findings highlight early promising evidence on some SCFs while adding to inconsistencies found in the broader health behavior literature.

Risk perception. The role of risk perceptions in physical activity initiation remains in question. In the present study it was not associated with behavioral intention or physical activity. As discussed previously, methodological challenges to the risk perception construct likely contributed to risk perception's consistent inconsistency in both this study and the health behavior literature (Schwarzer et al., 2003; Schwarzer, 2008). Yet even with sound psychometric measures, risk perception may simply not affect the formation of behavioral intention or behavior change itself in certain (or many) behaviors. This may seem exceptionally true for lifestyle behaviors such as diet and physical activity, as the consequence for failing to make lifestyle behavior changes are 
extremely distal. For many individuals with type 2 diabetes, decades may pass before diabetes-related complications become evident. This is in stark contrast to acute symptoms of pain or illness that would likely influence risk perceptions to formulate action-oriented behaviors, such as seeking medical attention.

Clinical implications directly relate to DSME curriculum, as it often emphasizes the costs of not engaging in appropriate diabetes self-care. With respect to physical activity, if such teaching affects risk perception, but risk perception fails to influence behavioral intention and subsequently behavior change itself, perhaps DSME should shy away from emphasis on health promoting messages. The alternative would enable a greater amount of time developing and implementing strategies to translate intention into action and addressing barriers to behavior change. This conclusion would tend to be supported by the broader health behavior theory literature discussed above.

Outcome expectancies. The role of outcome expectancy in physical activity initiation in the DSME population remains clouded. While the present study revealed positive outcome expectancies predicted behavioral intention, their contribution was relatively weak compared to that of self-efficacy. This finding is not unique in the broader health behavior literature and reasons for this have been cited as mainly a reflection of methodological concerns, including whether studies should include both positive and negative outcome expectancies (Williams et al., 2005; Williams et al., 2008). However, the present study's finding reflects the HAPA model's formulation and its underlying premise of social-cognitive theory that posits outcome expectancies in general predict the formation of self-efficacy, in contrast to behavioral intention (Bandura, 1997; Schwarzer, 1992; Schwarzer et al., 2003; Schwarzer, 2008). Future studies within the 
DSME population incorporating the HAPA framework should explicitly examine the predictive role of outcome expectancies on both self-efficacy and behavioral intention concurrently.

Self-efficacy. The present study dichotomized self-efficacy into stage specific self-efficacious beliefs based on the HAPA model's formulation of a motivational and volitional stage. Consistent with the HAPA literature, task self-efficacy was a prominent predictor of behavioral intention. In contrast, barrier self-efficacy failed to predict (i.e., increase the odds) that a patient would meet physical activity recommendations. The present study's findings suggest that the importance of barrier self-efficacy to physical activity initiation remains unclear in the DSME population, despite empirical support in cardiac and orthopedic rehabilitation populations (Blanchard, Courneya, et al., 2002; Blanchard, Rodgers, et al., 2002; Scholz et al., 2005; Schwarzer, 2008; Schwarzer \& Renner, 2000). One possible implication for these findings may be the need to examine the role of task self-efficacy on actual behavior. Some HAPA model formulations suggest task self-efficacy predicts behavior itself via (i.e., mediated by) barrier self-efficacy (Schwarzer, 2008). As there was no existing data prior to the present study on this relationship within the DSME populations, this alternative pathway of task self-efficacy was not tested. Future empirical investigations should explore the potential mediated relationship between task and barrier self-efficacy predicting physical activity in the DSME population. The support for task self-efficacy alone suggests that diabetes educators should promote interventions to increase patient's self-efficacious beliefs to improve subsequent engagement in physical activity. 
Behavioral intention. The present study contributed to a small but promising literature of social-cognitive predictors of physical activity in diabetes populations (Omondi et al., 2010; Plotnikoff et al., 2010). Knowledge of a patient's intention (or lack thereof) to engage in physical activity enables diabetes educators and healthcare providers alike to prioritize interventions. Applied to DSME, these findings suggested that for those patients with intentions to engage in physical activity, clinical efforts should be focused on assisting patients in transforming intentions into action. For those without such intentions, efforts can be focused on clarifying the patient's outcome expectancies and task self-efficacy in the hopes of moving a patient closer to engaging in physical activity. The challenge for healthcare providers and diabetes educators lies in translating these intentions into actual behavior - the notorious intention-behavior gap (Schwarzer, 2008; Sutton, 2008).

Action planning. Action planning failed to yield a direct or indirect effect on physical activity in the present study. One of the key issues with this finding may be what was not measured in the present study, namely, coping planning. Coping planning is conceptualized as a means of applying cognitive strategies to managing barriers that may interfere with ongoing behavior (Schwarzer et al., 2003; Schwarzer, 2008). As such, this construct was omitted in the design of the present study since it was assumed a priori that patients in DSME would be initiating physical activity, rather than maintaining the behavior. Future studies examining the planning construct in the DSME population should include both action and coping planning as a means to determine if patients in DSME have begun formulating coping plans. While the present study did not support the role of action plans in predicting physical activity, current DSME curriculum provides 
some, albeit limited, instruction in basic planning strategies. Expansion of these topics would benefit theoretical processes such as action or coping planning, as well as facilitate a larger portion of DSME time to physical activity, thereby likely affecting other socialcognitive constructs such as self-efficacy, outcome expectancies, and behavioral intention.

The present study yielded early information on the predictive role and function of SCFs as they pertain to physical activity initiation in DSME. However, it was their conceptualization within the broader framework of a health behavior theory that offers promise for future empirical endeavors and clinical applications.

\section{HAPA Framework Applied to DSME and Type 2 Diabetes}

The Health Action Process Approach was selected as the health behavior theory for the present study due to its parsimonious conceptualization, its prominence at the forefront of empirical interest in current research, and the general lack of evidence of the HAPA within the DSME and type 2 diabetes populations. It is recognized by this author, as well as others in the empirical literature, that there are other prominent social-cognitive factors (e.g., social norms) and other models (e.g., Theory of Planned Behavior) that have contributed to the prediction of physical activity in chronic illness populations (Armitage \& Conner, 2000; Schwarzer, 2008). As such, the HAPA framework is just one of several theories that could enhance understanding of physical activity initiation in DSME and type 2 diabetes populations.

In the present study, the HAPA model's motivational stage comprised of behavioral intentions formed by both task self-efficacy and positive outcome expectancies, with task self-efficacy playing a larger role, consistent with the HAPA 
conceptualization. With respect to the volitional stage, only behavioral intention predicted whether participants engaged in physical activity at the minimum recommended level. This finding was contrary to the HAPA model's formulation and suggested that behavioral intention may have been the most proximal predictor of behavior, a finding consistent with the Theory of Planned Behavior. Recent evidence has supported the Theory of Planned Behavior's utility in this population (Omondi et al., 2010; Plotnikoff et al., 2010). Does this suggest that the Theory of Planned Behavior would be better suited to predict physical activity initiation in type 2 diabetes? Not necessarily, as recently published data suggests the possible presence of more complex relationships than originally posited by the HAPA model, such as moderated-mediation of the intention-behavior gap (Lippke et al., 2009; Scholz et al., 2008; Wiedemann et al., 2009).

With the present study being the first to examine the HAPA within a type 2 diabetes and DSME population, it remains to be seen whether behavioral intention truly is the most proximal predictor for physical activity initiation, or whether other intermediate SCFs (such as coping planning) may present as key factors. Despite some inconsistent findings, overall early evidence from the present study suggests SCFs, as conceptualized by the HAPA model, may play an important role in physical activity initiation within the DSME population.

\section{Summary}

The present study's findings reveal a challenging duality facing health behavior theorists and diabetes health care providers. On one side, the challenge remains to establish scientifically rigorous, theoretically-driven evidence for promoting physical 
activity initiation early in DSME. On the other side, the challenge remains to translate the scientific evidence into the logistically restrictive context in which DSME operates. Noting time and financial constraints on DSME educators and patients alike, the need to identify parsimonious interventions that yield effective and timely results remains a high priority. While striking a balance between these two unique positions will remain a challenge for the foreseeable future, the present study's findings are a first step to improving physical activity behavior in the DSME population.

\section{General Strengths and Limitations}

\section{Strengths of the Present Study}

Study Design. The present study had several strengths related to its study design. These included using a theory-driven, longitudinal design and the use of a representative sample of participants with very little attrition across the duration of the study.

Theory-driven. Pivotal to the understanding of social-cognitive factors and how they may affect behavior is their empirical exploration within the greater framework of a theory. The empirical literature is wrought with studies in which SCFs, usually selfefficacy, is touted as important without any theoretical explanation; unfortunately the diabetes literature is not immune to this (American Association of Diabetes Educators, 2009a; Lippke \& Ziegelmann, 2008). The present study's use of the HAPA model as an overarching framework allowed for the assessment of potential social-cognitive predictors of physical activity while providing insight as to why such SCFs might predict such behavior.

Longitudinal design. The present study's prospective, longitudinal design yielded key information regarding physical activity patterns in patients with type 2 diabetes as 
they moved through DSME. Moreover, this descriptive information revealed the proportion of patients who met the minimum physical activity recommendations.

Although this study was correlational, its longitudinal design improved the amount of inference that could be drawn from regression analyses on physical activity. For example, since the dependent variable was measured at a date later than the theorized predictors, temporal precedence could be established (Cohen et al., 2003).

Sampling. A convenience sample was used for the present study. In general, with exception to gender, this sample was very similar to the socio-demographic characteristics of the surrounding metropolitan area (U.S. Census Bureau, 2010). Additionally, while initial recruitment of study participants remained a challenge throughout the duration of the study, attrition between study waves remained relatively low. Of those who did drop out, differences between completers and non-completers were few and not related to the study's primary outcomes.

Expansion of a limited literature. A clear strength of this study was its expansion of an extremely limited empirical literature. Findings in the present study lay the ground work for future scientific exploration in the type 2 diabetes and more specifically, DSME populations. Specifically, the findings in this study helped clarify and inform which social-cognitive factors may play a key role in predicting physical activity initiation. Coupled with the use of a health behavior theory, this study enabled both the identification of SCFs and facilitated elaboration on how these factors may have influenced physical activity initiation. Additionally, this study contributed to the extremely limited data on physical activity rates in DSME. 


\section{Limitations of the Present Study}

In addition to the limitations to the specific hypotheses addressed above, the present study had several general limitations that limit the interpretation and generalizability of the current findings. These limitations were predominantly methodological yet serve to remind the reader of the delicate balance between scientific rigor and study feasibility.

Correlational design. Consistent with most of the available health behavior theory literature, the present study used a correlational design to model relationships among social-cognitive factors and their ability to predict behavior. The hallmark limitation to this falls back on the adage of correlation does not equal causation. As such, causal inferences drawn from this study must be restricted to merely informing directions for future controlled, experimental studies.

Self-report data. Perhaps the most widely used and financially affordable modalities for collecting data, the use of self-report measures are limited by their subjective nature. The present study's use of self-report measures, while consistent with the broader empirical literature, limits the reliability and validity of the findings. This limitation was reflected in two particular categories, (a) physical activity measurement, and (b) social-cognitive measurement. As previously noted, the use of physical activity self-report data can be heavily skewed and biased by memory, social-desirability, overreporting, etc. Despite this, a recent meta-analysis of both subjective self-report and objective measures of physical activity revealed both types of measures were susceptible to under- and over-reporting bias (Prince et al., 2008). As such, evidence suggests there are drawbacks to both self-report and objective measures of physical activity. With 
respect to measurement of social-cognitive factors, inference drawn from the present study's findings are limited by the psychometric properties of these measures. Since the measurement of SCFs is limited to self-report and specific behavior and population domains, the lack of gold standard measures represents a significant barrier to the evolution of current health behavior theory.

Measurement error. Measurement error may have affected findings of certain SCFs (e.g., risk perception, outcome expectancies) in the present study, Limitations in the measurement of risk perception appear related to a broader conceptual debate in the literature. Conversely, limitations to the measurement of outcome expectancies appears more related to a lack of a gold standard across studies, even within the same research domain (Williams et al., 2005). In the present study, the positive outcome expectancies measure yielded fair to sub-par internal consistency which may have affected its utility. Perhaps the most prominent measure with potential error in the present study was the IPAQ. As recent evidence has suggested its potential for over-reporting bias, it remains unclear whether self-reported scores in this study's sample were both reliable and valid (Bauman et al., 2009). It was also unclear why the telephone-based IPAQ demonstrated poorer sub-scale internal consistency compared to the self-administered forms. One possibility may have been related to the IPAQ's tendency to yield heavily skewed data . Greer, Dunlap, Hunter, and Berman (2006) examined the effects of skew on standardized item alphas, which were used to assess the IPAQ sub-scale reliabilities. Their findings suggested that skewed data could decrease inter-item correlations, which could potentially explain the lowered internal consistency on the IPAQ L/T in the present 
study. Without knowing the exact cause, these limitations suggest caution when interpreting and applying findings in the present study.

Statistical limitations. Closely related to limitations of measurement error, statistical limitations were present in this study due to the extreme non-normal distribution of data measured by the IPAQ. This non-normal distribution required a posthoc adjustment to this study's data analytic plan. The present study's shift from linear regression to logistic regression made relative comparisons with the current HAPA literature difficult. In particular, logistic regression does not necessarily allow for one to identify the amount of variance accounted for by the regression model. Future studies in the DSME setting may face this difficulty if the physical activity patterns observed in the present study were representative of the broader DSME population.

Potential sampling bias. A key limitation to the present study was reflected in the over-representation of women in the present sample. Women represented $73.2 \%$ of this sample compared to $26.8 \%$ men. The sampling bias towards women may reflect more of a systematic phenomenon in the DSME population. Women are often more likely than men to attend DSME (Gucciardi et al., 2008). The generalizability of this study's findings is likely limited to female patients with type 2 diabetes attending DSME.

Another potential source of sampling bias in the present study was related to this sample's high level of education, with most $(>60 \%)$ of participants with some form of post-high school education. Comparisons with the surrounding metropolitan area were limited as Census data for the year 2010 was not yet available and the last available comparative figures were from 2000 . While this may have reflected an overrepresentation of higher educated adults, it was unclear to what degree. 
Lengthy data collection. The final limitation of the present study was related to the amount of time necessary to obtain the study's longitudinal sample. Spanning approximately 17 months, the present study revealed significant challenges to subject recruitment. Once participants entered the study, attrition was kept low. However, the amount of time needed to acquire 152 cases with three complete Waves of data highlight specific challenges to conducting research in this population. The reasons for such slow recruitment are pure speculation and could not be confirmed, yet serve as a "food for thought" for future researchers interested in studying lifestyle behavior change in the DSME population.

\section{Final Summary}

In this prospective, longitudinal study, social-cognitive determinants of physical activity were studied in people with type 2 diabetes participating in Diabetes SelfManagement Education. Findings of this study revealed that several key social-cognitive factors, as conceptualized by the Health Action Process Approach, predicted both the formation of behavioral intention to engage in physical activity, as well as which participants met the minimum amounts of physical activity promoted in DSME. Findings suggest that the HAPA model provides a useful framework for understanding the functional roles of these social-cognitive constructs, while highlighting the need for future research in this population to best understand how to translate intention into actual behavior change.

Additionally, the present study revealed physical activity patterns of DSME participants up to eight weeks after learning the specific physical activity guidelines. Future directions for research should include controlled, experimental designs to establish 
the causal role of social-cognitive factors in physical activity initiation. Also, expansion of the present study through the use of more power statistical tests may allow better understanding of the inter-relationships among the social-cognitive factors and their relationship with physical activity. Clinical implications for these findings are highlighted by the considerable work that remains to improve rates of physical activity initiation in the DSME population. The pandemic of type 2 diabetes presents a significant challenge to researchers and clinicians alike. Furthering empirical understanding and clinical application of these findings should become a public health priority if the projected prevalence of type 2 diabetes and its debilitating complications are to be restrained. 
Table 1

Factors that Influence Initiation \& Maintenance of Physical Activity

General Population $^{\text {a }}$ Specific to T2DM ${ }^{\mathrm{a}}$

Physical Activity History Hyper-/Hypoglycemia

BMI

Neuropathy

Health Risk Profile

Lower-limb Amputation

Smoking, ETOH use

Medication/Insulin Side Effects

Stress / Psychological Distress

Fatigue

Depression

Gender/Age/Ethnicity

Socio-economic status

Time

Access to exercise facilities

Chronic pain

Social-cognitive processes

Note. T2DM: type 2 diabetes mellitus; BMI: body mass index; ETOH: alcohol.

a Adapted from: (American Diabetes Association, 2002; Sherwood \& Jeffery, 2000; Thomas et al., 2004). 
Table 2

Overlap of Key Social-Cognitive Constructs Across Health Behavior Models

Social-Cognitive Construct

Health Behavior Model (Model Construct)

Risk Perception

HBM (Perceived Threat)

HBM (Cues to Action)

TPB (Normative Beliefs)

TTM (Contemplation Phase)

Outcome Expectancies

HBM (Benefits vs. Barriers)

PMT (Response Efficacy)

TPB (Behavioral Beliefs)

TTM (Decisional Balance)

Self-Efficacy

HBM (Self-Efficacy)

PMT (Self-Efficacy)

TTM (Self-Efficacy)

TPB (Perceived Behavioral Control)

Behavioral Intention

PMT (Intention)

TPB (Behavioral Intention)

Note. HBM: Health Belief Model; TTM: Transtheoretical Model; PMT: Protection Motivation Theory; TPB: Theory of Planned Behavior. 
Table 3

Data Collection Sites with Sample Size and Diabetes Education Class Format

\begin{tabular}{ccccc}
\hline Site & $\begin{array}{c}\text { Completers } \\
n(\%)\end{array}$ & $\begin{array}{c}\text { Non-Completers } \\
n(\%)\end{array}$ & $\begin{array}{c}\text { Site } \\
\text { Type }^{\mathrm{a}}\end{array}$ & $\begin{array}{c}\text { Class } \\
\text { Format }^{\mathrm{b}}\end{array}$ \\
\hline 1 & $33(21.7)$ & $18(31.0)$ & Primary & $\mathrm{M}$ \\
2 & $26(17.1)$ & $13(22.4)$ & Primary & $\mathrm{S}$ \\
3 & $6(3.9)$ & $3(5.2)$ & Secondary & $\mathrm{S}$ \\
5 & $52(34.2)$ & $11(19.0)$ & Primary & $\mathrm{M}$ \\
6 & $6(3.9)$ & $0(0)$ & Secondary & $\mathrm{M}$ \\
& $29(19.1)$ & $13(22.4)$ & Primary & $\mathrm{M} / \mathrm{S}$ \\
\hline
\end{tabular}

Note. Site 1: Clark Memorial Hospital, Indiana; Site 2: Norton Healthcare, Louisville Metro area; Site 3: Baptist Hospital East, Louisville; Site 4: Jewish Hospital East / St. Mary \& Elizabeth Hospitals, Louisville; Site 5: Louisville Metro Public Health Department; Site 6: Joslin Diabetes Center at Floyd Memorial Hospital, Indiana; M: Multi-Session; S: Single Session

${ }^{\text {a }}$ Primary sites accounted for the majority of recruitment invites ( $95 \%$ of 720 invites).

${ }^{\mathrm{b}}$ Multi-session/Single Session: Curriculum taught across several weeks versus one session, respectively. 
Table 4

Data Collection Waves, Timeline \& Construct Measurement

\begin{tabular}{|c|c|c|c|}
\hline Wave & $\begin{array}{l}\text { Time } \\
M(S D)^{\mathrm{a}}\end{array}$ & Method of Administration & Constructs Measured \\
\hline \multirow[t]{4}{*}{1} & Baseline & Self-Administered & Demographics, Diabetes \\
\hline & & & History, HAPA constructs, \\
\hline & & & Depressive Symptomology, \\
\hline & & & Physical Activity \\
\hline \multirow[t]{2}{*}{2} & $27.93(9.9)$ & Self-Administered & HAPA constructs, Physical \\
\hline & & & Activity \\
\hline \multirow[t]{2}{*}{3} & $22.76(6.36)$ & Telephone (Interviewer) & Physical Activity, Cognitive \\
\hline & & Administered & Screening ${ }^{b}$ \\
\hline
\end{tabular}

Note. HAPA: Health Action Process Approach.

${ }^{a} \mathrm{M}(\mathrm{SD})$ : Mean and Standard Deviation in days between each wave; e.g., Wave 2 Time reflects average number of days between the Wave 1 and Wave 2 measurements.

${ }^{\mathrm{b}}$ Cognitive screening administered to participants 60 years and older $(n=49)$. 
Table 5

Psychological \& Physical Activity Study Measures with Associated Constructs

Study Measure

Diabetes Health Beliefs

Opinions about Exercise

Confidence in Exercise

Exercise Intention

Confidence in Overcoming

Exercise Barriers

Exercise Planning

Center for Epidemiologic Studies -

Short Depression Scale

Telephone Interview for Cognitive

Status

International Physical

Activity Questionnaire
Construct

Risk Perception

Positive Outcome Expectancies

Task Self-Efficacy

Behavioral Intention

Barrier Self-Efficacy

Action Planning

Depressive Symptomology

Mental Status

Physical Activity 
Table 6

Summary of Reliability Statistics on Study Measures

\begin{tabular}{|c|c|c|c|c|c|}
\hline \multirow[t]{3}{*}{ Construct } & \multirow[t]{3}{*}{ Measure } & \multicolumn{4}{|c|}{ Reliability Statistics ${ }^{a}$} \\
\hline & & \multicolumn{3}{|c|}{ Internal Consistency $^{\mathrm{b}}$} & \multirow[t]{2}{*}{ Test-Retest ${ }^{c}$} \\
\hline & & Wave 1 & Wave 2 & Wave 3 & \\
\hline $\mathrm{RP}$ & $\begin{array}{l}\text { Diabetes Health } \\
\text { Beliefs }\end{array}$ & .66 & .74 & & .60 \\
\hline $\mathrm{OE}$ & $\begin{array}{l}\text { Opinions About } \\
\text { Exercise }\end{array}$ & .30 & .54 & & .40 \\
\hline $\operatorname{SE}(\mathrm{t})$ & $\begin{array}{l}\text { Confidence in } \\
\text { Exercise }\end{array}$ & .94 & .94 & & .61 \\
\hline BI & Exercise Intention & .73 & .81 & & .57 \\
\hline $\operatorname{SE}(b)$ & $\begin{array}{l}\text { Confidence in } \\
\text { Overcoming } \\
\text { Barriers }\end{array}$ & .93 & .93 & & .70 \\
\hline $\mathrm{AP}$ & Exercise Planning & .90 & .90 & & .51 \\
\hline \multirow{4}{*}{$\begin{array}{l}\text { IPAQ } \\
\text { Domain } \\
\text { Scales }^{d}\end{array}$} & Work Sub-scale & .90 & .90 & .78 & .52 \\
\hline & $\begin{array}{c}\text { Transportation } \\
\text { Domain Sub-Scale }\end{array}$ & .54 & .61 & .51 & .25 \\
\hline & $\begin{array}{c}\text { Domestic \& } \\
\text { Garden Sub-Scale }\end{array}$ & .72 & .70 & .38 & .51 \\
\hline & $\begin{array}{l}\text { Leisure-Time Sub- } \\
\text { Scale }\end{array}$ & .72 & .65 & .46 & .40 \\
\hline
\end{tabular}




\begin{tabular}{|c|c|c|c|c|c|}
\hline \multirow[t]{3}{*}{ Construct } & \multirow[t]{3}{*}{ Measure } & \multicolumn{4}{|c|}{ Reliability Statistics } \\
\hline & & \multicolumn{3}{|c|}{ Internal Consistency $^{b}$} & Test-Retest $^{\mathrm{c}}$ \\
\hline & & Wave 1 & Wave 2 & Wave 3 & \\
\hline $\begin{array}{l}\text { Depressive } \\
\text { Symptoms }\end{array}$ & CES-D 10 & .83 & & & \\
\hline
\end{tabular}

Note: RP: risk perception, OE: positive outcome expectancies, $\mathrm{SE}(\mathrm{t})$ : task self-efficacy, BI: behavioral intention, SE(b): barrier self-efficacy, AP: action planning; IPAQ: International Physical Activity Questionnaire; CES-D 10: Center for Epidemiologic Studies Short Scale

${ }^{a}$ All $n$ 's $=152$ (completers only), except: Wave $1 \mathrm{RP}(n=150)$, Wave $1 \mathrm{SE}(\mathrm{t})(n=151)$, Wave 1 and 2 $\operatorname{SE}(\mathrm{b})(n$ 's $=146$ and 148, respectively), and CES-D $10(n=148)$; all figures rounded to hundredths.

${ }^{b}$ All internal consistency statistics are Cronbach's Alpha, except IPAQ (see below)

' Test-retest data for time between Wave 1 and Wave 2 only, all statistics are Pearson's $r$, except IPAQ (see below).

dStandardized Item Cronbach's Alpha reported for IPAQ subscales due to different scaling within each sub-scale (e.g., time versus days within the same scales). Spearman's rho statistics used for IPAQ testretest correlations. 
Table 7

Summary of Recommended Classifications of Physical Activity

Category

Low

Moderate

High
OR

OR

3 or more days of vigorous-intensity activity of at least 20-minutes per day.

Lowest level of physical activity; Individuals who do not meet Moderate or High Categories.

5 or more days of moderate-intensity activity and/or walking of at least 30 minutes per day.

5 or more days of any combination of walking, moderate-intensity, or vigorous intensity activities achieving a minimum total physical activity of at least 600 MET-minutes per week. ${ }^{a}$

Vigorous-intensity activity on at least 3 days achieving a minimum total physical activity of at least $1500 \mathrm{MET}$ minutes per week.

OR

7 or more days of any combination of walking, moderateintensity, or vigorous-intensity activities achieving a minimum total physical activity of at least 3000 METminutes per week.

Note. From (Sjostrom et al., 2005). MET: metabolic equivalents.

${ }^{a}$ Formerly known as the leisure-time physical activity recommendation, this is consistent with current American Diabetes Association and American College of Sports Medicine recommendations for the minimum amount of weekly physical activity taught in diabetes education. 
Table 8

Socio-Demographic Characteristics of Participants (Completers Only)

\begin{tabular}{|c|c|c|}
\hline Variable & Frequency & $\%$ \\
\hline Age & & \\
\hline Gender & & \\
\hline Female & 109 & 73.2 \\
\hline Male & 40 & 26.8 \\
\hline
\end{tabular}

Ethnicity

$\begin{array}{lcc}\text { Caucasian } & 123 & 82.6 \\ \text { African American } & 24 & 16.1 \\ \text { Hispanic } & 1 & 0.7 \\ \text { Other } & 1 & 0.7\end{array}$

Marital Status

Never Married

Married

Separated

Divorced

Widowed

Current Living Arrangement

Live alone

Live w/spouse/partner

Live w/spouse/partner and children

$\begin{array}{lll}148 & 53.9 & 11.6\end{array}$

149

n $\quad M \quad S D$

49




\begin{tabular}{|c|c|c|c|c|c|}
\hline$\overline{\text { Variable }}$ & Frequency & $\%$ & $n$ & $M$ & $S D$ \\
\hline $\begin{array}{l}\text { Live w/children } \\
\text { (no spouse/partner) }\end{array}$ & 14 & 9.4 & & & \\
\hline $\begin{array}{l}\text { Live with roommate } \\
\text { who is not partner }\end{array}$ & 2 & 1.3 & & & \\
\hline Live with parents & 2 & 1.3 & & & \\
\hline $\begin{array}{l}\text { Other living } \\
\text { arrangement }\end{array}$ & 7 & 4.7 & & & \\
\hline $\begin{array}{l}\text { Highest Education Level } \\
\text { Attained }\end{array}$ & & & 149 & & \\
\hline $\begin{array}{l}\text { Partial High School } \\
\text { or less }\end{array}$ & 11 & 7.4 & & & \\
\hline High School Grad & 46 & 30.9 & & & \\
\hline $\begin{array}{l}\text { Some College/Trade } \\
\text { school }\end{array}$ & 39 & 26.2 & & & \\
\hline College Graduate & 34 & 22.4 & & & \\
\hline Graduate Degree & 19 & 12.8 & & & \\
\hline Annual Household Income & & & 142 & & \\
\hline Less than $\$ 10,000$ & 14 & 9.9 & & & \\
\hline$\$ 10,000-\$ 19,999$ & 18 & 12.7 & & & \\
\hline$\$ 20,000-\$ 39,999$ & 41 & 28.9 & & & \\
\hline$\$ 40,000$ - $\$ 59,999$ & 29 & 20.4 & & & \\
\hline$\$ 60,000-\$ 100,000$ & 23 & 16.2 & & & \\
\hline Over $\$ 100,000$ & 17 & 12.0 & & & \\
\hline
\end{tabular}




\begin{tabular}{cccccc}
\hline Variable & Frequency & $\%$ & $n$ & $M$ & $S D$ \\
\hline Current Employment Status & & & 150 & & \\
Working full time & 66 & 44.0 & & & \\
Working part time & 14 & 9.3 & & \\
On leave with pay & 1 & 0.7 & & \\
On leave without pay & 1 & 0.7 & & \\
Disabled & 20 & 13.3 & & \\
Seeking work & 7 & 4.7 & & \\
Retired & 33 & 22.0 & \\
Homemaker & 7 & 4.7 & \\
Student & 1 & 0.7 & \\
\hline
\end{tabular}


Table 9

Percentage Comparison of Study Sample to Local, State, and National SocioDemographic Characteristics

\begin{tabular}{|c|c|c|c|c|}
\hline Characteristic & $\begin{array}{l}\text { Study } \\
\text { Sample }\end{array}$ & $\begin{array}{l}2008^{a} \\
\text { Local }\end{array}$ & $\begin{array}{l}\text { State } \\
(\mathrm{KY})\end{array}$ & National \\
\hline Female & 73.2 & 51.85 & 51.1 & 50.7 \\
\hline \multicolumn{5}{|l|}{ Ethnicity } \\
\hline Caucasian & 82.6 & 80.4 & 89.9 & 79.8 \\
\hline African-American & 16.1 & 16.5 & 7.7 & 12.8 \\
\hline \multicolumn{5}{|l|}{ Education $^{\mathrm{b}}$} \\
\hline High School Grad & 92.6 & & 74.1 & 80.4 \\
\hline $\begin{array}{l}\text { College Grad or } \\
\text { Higher }\end{array}$ & 35.2 & & 17.14 & 24.4 \\
\hline $\begin{array}{l}\text { Annual Household } \\
\text { Income }^{c}\end{array}$ & $<39,999$ & 45,050 & 41,538 & 52,029 \\
\hline
\end{tabular}

Note. All data from (U.S. Census Bureau, 2010)

a Combined (averaged) 2008 estimates from Louisville-Metro, Jefferson County (Kentucky), Floyd County (Indiana) and Clark County (Indiana).

${ }^{\mathrm{b}} 2000$ Estimates.

${ }^{c}$ Annual Household Income is defined as $<50 \%$ of study sample at 39,999 or less; other values reported are median income for 2008 
Table 10

BMI, Depressive Symptomology, and Diabetes-Related Characteristics of Participants

\begin{tabular}{llllll}
\hline Variable & Frequency & $\%$ & $n$ & $M$ & $S D$ \\
\hline
\end{tabular}

Body Mass Index

Body Mass Index

Classifications $^{\text {a }}$

Normal

Pre-Obese

Class I Obesity

Class II Obesity

Class III Obesity

CES-D $10^{b}$

Not Depressed

Possible Depression

Duration Diagnosed with

Type 2 Diabetes Mellitus in Months

1 Month or Less

Greater than 1 Month

Insulin Use

Yes

26

126

6

44

84

65

54

98

No

$\begin{array}{lll}144 & 36.5 & 8.7\end{array}$

144

4.2

18.1

23.6

23.6

30.6

$\begin{array}{lll}149 & 9.77 \quad 6.01\end{array}$

56.4

43.6

$152^{\mathrm{c}}$

35.5

64.5

152

17.1

82.9 


\begin{tabular}{|c|c|c|c|c|c|}
\hline Variable & Frequency & $\%$ & $n$ & $M$ & $S D$ \\
\hline $\begin{array}{l}\text { Co-Occurring Health } \\
\text { Conditions } \\
\text { (\% Endorsed) }\end{array}$ & & & 152 & & \\
\hline Myocardial Infarction & 13 & 8.6 & & & \\
\hline Heart Failure & 7 & 4.6 & & & \\
\hline High Cholesterol & 103 & 67.8 & & & \\
\hline Angina & 15 & 9.9 & & & \\
\hline High Blood Pressure & 113 & 74.3 & & & \\
\hline CABG & 4 & 2.7 & & & \\
\hline Angioplasty & 6 & 3.9 & & & \\
\hline $\begin{array}{l}\text { Peripheral Vascular } \\
\text { Disease }\end{array}$ & 9 & 5.9 & & & \\
\hline $\begin{array}{l}\text { Intermittent } \\
\text { Claudication }\end{array}$ & 9 & 6.0 & & & \\
\hline $\begin{array}{l}\text { Peripheral } \\
\text { Neuropathy }\end{array}$ & 34 & 22.5 & & & \\
\hline $\begin{array}{l}\text { Cerebrovascular } \\
\text { Accident }\end{array}$ & 5 & 3.3 & & & \\
\hline $\begin{array}{l}\text { Transient Ischemic } \\
\text { Attack }\end{array}$ & 9 & 6.0 & & & \\
\hline
\end{tabular}

Note. All data reported is for completers only. CES-D 10: Center for Epidemiologic Studies Short Scale.

${ }^{a}$ Body Mass Index Classifications based on World Health Organization standards: (World Health

Organization, 2010).

${ }^{b}$ CES-D 10 cut-off score of $\geq 10$ used to identify probable depressive symptomology.

${ }^{\mathrm{c}}$ For Duration Diagnosed with Type 2 Diabetes Mellitus, median: 4 months and mode: 1 month or less. 


\section{Table 11}

Participant Leisure-Time Physical Activity Characteristics - Continuous Scores of Energy Expenditure

\section{Wave 1}

LT Walking Activity

LT Moderate-Intensity Activity

LT Vigorous-Intensity Activity

Total LT Physical Activity

Wave 2

LT Walking Activity

LT Moderate-Intensity Activity

LT Vigorous-Intensity Activity

Total LT Physical Activity

Wave 3

LT Walking Activity

LT Moderate-Intensity Activity 128.60

LT Vigorous-Intensity Activity

Total LT Physical Activity

Note. $N=152$ (completers only). LT=Leisure Time; MET: metabolic equivalents; Continuous scores for this sample were significantly positively skewed.

Median values for continuous scores presented as recommended by the International Physical Activity Questionnaire (IPAQ) Development Committee (Sjostrom et al., 2005). 
Table 12

Participant Levels of Leisure-Time Physical Activity

\begin{tabular}{lcll}
\hline Level of Activity & Study Wave & $\%$ & $n$ \\
\hline Low & 1 & 85.5 & 130 \\
& 2 & 78.3 & 119 \\
& 3 & 67.8 & 103 \\
& & & \\
Moderate & 1 & 14.5 & 22 \\
& 2 & 18.4 & 28 \\
& 3 & 30.3 & 46 \\
High & & & \\
& 1 & 0 & 0 \\
& 2 & 3.3 & 5 \\
\hline
\end{tabular}

Note. $N=152$; Categorical classifications of "Low, Moderate, High" defined per by the International Physical Activity Questionnaire (IPAQ) Development Committee (Sjostrom et al., 2005). See Table 7 for definitions. 
Table 13

Social-Cognitive Characteristics of Participants

\begin{tabular}{lcccc}
\hline Variable & $n$ & $M$ & $S D$ & Min-Max \\
\hline & & Wave 1 & & \\
& & & & \\
& 150 & 8.06 & 2.51 & $0-12$ \\
Risk Perception & 152 & 69.72 & 13.83 & $0-100$ \\
$\begin{array}{l}\text { Positive Outcome } \\
\text { Expectancies }\end{array}$ & 152 & 5.67 & 2.66 & $1-10$ \\
Task Self-Efficacy & 152 & 4.19 & 1.37 & $1-7$ \\
Behavioral Intention & 152 & 52.30 & 22.39 & $0-100$ \\
Barrier Self-Efficacy & 152 & 2.58 & 0.93 & $1-4$
\end{tabular}

Wave 2

\begin{tabular}{lcccc} 
Risk Perception & 152 & 8.00 & 2.75 & $0-12$ \\
$\begin{array}{l}\text { Positive Outcome } \\
\text { Expectancies }\end{array}$ & 152 & 71.84 & 15.01 & $0-100$ \\
$\begin{array}{l}\text { Task Self-Efficacy } \\
\text { Behavioral Intention }\end{array}$ & 152 & 5.76 & 2.56 & $1-10$ \\
Barrier Self-Efficacy & 152 & 4.20 & 1.51 & $1-7$ \\
Action Planning & 152 & 50.76 & 22.36 & $0-100$ \\
\hline
\end{tabular}

Note. Min-Max $=$ Scale minimum and maximum possible scores. Higher scores represent higher levels of social-cognitive factor on all measures. 
Table 14

Differences between Depression Status and Social-Cognitive Factors

\begin{tabular}{|c|c|c|c|c|c|}
\hline SCF & $M(S D)$ & $t$ & $p<$ & Mean Difference & $95 \% \mathrm{CI}$ \\
\hline W1OE & $\begin{array}{l}66.7(13.6) \dagger \\
71.9(13.6) \ddagger\end{array}$ & -2.33 & .05 & -5.22 & -9.65 to -.79 \\
\hline W1SEt & $\begin{array}{l}5.19(2.7) \dagger \\
6.10(2.6) \ddagger\end{array}$ & -2.03 & .05 & -.89 & -1.75 to -.02 \\
\hline W1BI & $\begin{array}{l}3.92(1.5) \dagger \\
4.42(1.3) \ddagger\end{array}$ & -2.24 & .05 & -.50 & -.94 to -.06 \\
\hline W1SEb & $\begin{array}{l}46.70(23.9) \dagger \\
56.75(20.5) \dagger\end{array}$ & -2.80 & .01 & -10.05 & -17.24 to -2.86 \\
\hline W1AP & $\begin{array}{l}2.40(.90) \dagger \\
2.72(.94) \dagger\end{array}$ & -2.32 & .05 & -.35 & -.65 to -.05 \\
\hline W2BI & $\begin{array}{l}3.91(1.6) \dagger \\
4.41(1.5) \ddagger\end{array}$ & -2.00 & .05 & -.50 & -.99 to -.005 \\
\hline W2SEb & $\begin{array}{l}46.12(23.8) \dagger \\
54.20(21.0) \dagger\end{array}$ & -2.20 & .05 & -8.05 & -15.30 to -.80 \\
\hline W2AP & $\begin{array}{l}2.60(.93) \dagger \\
2.91(.85) \dagger\end{array}$ & -2.32 & .05 & -.34 & -.63 to -.05 \\
\hline
\end{tabular}

Note. SCF: social-cognitive factor, W1OE:Wave 1 Positive Outcome Expectancies, W1SEt:Wave 1 Task Self-Efficacy, WIBI: Wave 1 Behavioral Intention, W1SEb:Wave 1 Barrier Self-Efficacy, W1AP:Wave 1 Action Planning, W2BI: Wave 2 Behavioral Intention, W2SEb:Wave 2 Barrier Self-Efficacy, W2AP:

Wave 2 Action Planning. Degrees of freedom $=147$ for all analyses.

$\dagger$ : Depression status: Probable $(n=65)$

\$: Depression status: Not depressed $(n=85)$ 
Table 15

Differences between Annual Household Income and Social-Cognitive Factors

\begin{tabular}{|c|c|c|c|c|c|}
\hline $\mathrm{SCF}$ & $M(S D)$ & $t$ & $p<$ & Mean Difference & $95 \% \mathrm{CI}$ \\
\hline W1RP & $\begin{array}{l}7.70(2.3) \dagger \\
8.53(2.5) \ddagger\end{array}$ & -2.06 & .05 & -.83 & -1.62 to -.03 \\
\hline W1SEt & $\begin{array}{l}6.4(2.6) \dagger \\
5.0(2.6) \ddagger\end{array}$ & 3.30 & .001 & 1.42 & .60 to 2.3 \\
\hline W2RP & $\begin{array}{l}7.34(2.7) \dagger \\
8.70(2.7) \ddagger\end{array}$ & -2.90 & .01 & -1.34 & -2.23 to -.45 \\
\hline W2OE & $\begin{array}{l}75.1(12.5) \dagger \\
69.6(17.1) \ddagger\end{array}$ & 2.16 & .05 & 5.5 & .47 to 10.5 \\
\hline W2SEt & $\begin{array}{l}6.40(2.5) \dagger \\
5.22(2.6) \ddagger\end{array}$ & 2.70 & .01 & 1.14 & .30 to 2 \\
\hline W2BI & $\begin{array}{l}4.60(1.5) \dagger \\
3.90(1.5) \ddagger\end{array}$ & 2.7 & .01 & .68 & .18 to 1.18 \\
\hline $\mathrm{W} 2 \mathrm{SEb}$ & $\begin{array}{l}56.01(22.5) \dagger \\
46.30(22.3) \dagger\end{array}$ & 2.6 & .05 & 9.71 & 2.29 to 17.17 \\
\hline
\end{tabular}

Note, SCF: social-cognitive factor, W1RP: Wave 1 Risk Perception, W1SEt:Wave 1 Task Self-Efficacy, W2RP: Wave 2 Risk Perception, W2OE: Wave 2 Outcome Expectancies, W2SEt: Wave 2 Task SelfEfficacy, W2BI: Wave 2 Behavioral Intention, W2SEb:Wave 2 Barrier Self-Efficacy. Degrees of freedom $=140$ for all analyses except W1RP $(d f=139)$.

$\dagger$ : Annual Household Income status: $\geq \$ 40,000(n=69)$, except for W1RP $(n=68)$

‡: Annual Household Income status: $\leq \$ 39,000(n=73)$ 
Table 16

Intercorrelations between 42 Dependent Variable, Independent Predictors and Covariates

\begin{tabular}{|c|c|c|c|c|c|c|c|c|}
\hline Variable & 1 & 2 & 3 & 4 & 5 & 6 & 7 & 8 \\
\hline 1. $\mathrm{W}_{2} \mathrm{BI}^{+}$ & 1.00 & & & & & & & \\
\hline 2. $\mathrm{W}^{2} \mathrm{OE}^{\dagger}$ & $.435^{* * *}$ & 1.00 & & & & & & \\
\hline 3. $\mathrm{W} 2 \mathrm{SEt}^{\dagger}$ & $.797^{* * *}$ & $.393^{* * *}$ & 1.00 & & & & & \\
\hline 4. $\mathrm{W} 1 \mathrm{BI}^{+}$ & $.641^{* * *}$ & $.399^{* * *}$ & $.650^{* * *}$ & 1.00 & & & & \\
\hline 5. DEPRESS ${ }^{\dagger}$ & $-.200^{*}$ & -.118 & $-.227^{* *}$ & -.126 & 1.00 & & & \\
\hline 6. W2LTPA ${ }^{\ddagger}$ & $.476^{* * *}$ & .051 & $.466^{* * *}$ & $.340^{* * *}$ & $-.180^{\circ}$ & 1.00 & & \\
\hline 7. DURDX ${ }^{\ddagger}$ & $-.202^{*}$ & -.051 & -.134 & -.056 & .110 & -.094 & 1.00 & \\
\hline 8. INCOME & $.257^{* *}$ & $.195^{*}$ & $.236^{* *}$ & .170 & $-.296^{* *}$ & $.186^{\circ}$ & -.134 & 1.00 \\
\hline
\end{tabular}

Note. Dependent variable: W2BI: Wave 2 Behavioral Intention; independent predictors: W2OE:Wave 2 Positive Outcome Expectancies, W2SEt:Wave 2 Task Self-Efficacy; covariates: W1BI:Wave 1 Behavioral, Depress: Depression status score; W2LTPA: Wave 2 Leisure-time Physical Activity, DURDX: Duration since diabetes diagnosis, INCOME $=$ annual household income

Listwise deletion for all variables yielded $n=131$.

${ }^{*} p<.05,{ }^{* *} p<.01,{ }^{* * *} p<.001$

$\dagger$ : Pearson's $r$

\#: Spearman's rho 
Table 17

H2 Regression Coefficients, Standard Errors, $\beta$ Weights, and Confidence Intervals for Model 2 (Full Model)

\begin{tabular}{ccc}
$b$ & Std Error $\quad$ & $\begin{array}{c}95 \% \mathrm{CI} \text { of } \\
b\end{array}$ \\
\hline
\end{tabular}

Block 2

W2OE

.011

.006

.113

.00 to .02

.046

W2SEt

.333

.042

.561

.25 to .42

$<.001$

Block 1

$\begin{array}{llllll}\text { W1BI } & .204 & .071 & .185 & .06 \text { to } .34 & .005 \\ \text { W2LTPA } & .444 & .209 & .122 & .03 \text { to } .90 & .035 \\ \text { DEPRESS } & .003 & .014 & .009 & -.03 \text { to } .03 & \text { NS } \\ \text { INCOME } & .104 & .165 & .034 & .43 \text { to } .23 & \text { NS } \\ \text { DURDX } & -.237 & .163 & -.073 & -.60 \text { to } .10 & \text { NS }\end{array}$

Note. $n=131$; Dependent variable: Wave 2 Behavioral Intention. Block 2 predictors: W2OE: Wave 2 Positive Outcome Expectancies, W2SEt: Wave 2 Task Self-Efficacy; Block 1 covariates: W1BI: Wave 1 Behavioral Intention, W2LTPA: Wave 2 Leisure-time Physical Activity, Depress: Depression score, INCOME: annual household income, DURDX: Duration since diabetes diagnosis. $b=$ unstandardized regression coefficient, Std Error: Standard Error of $b ; \beta$ : standardized regression coefficient, $\mathrm{CI}$ : Confidence Interval, NS: not statistically significant. All variables continuous scores except W2LTPA, DEPRESS, INCOME, DURDX, which are dummy (binary) coded categorical scores. 
Table 18

Intercorrelations between $H 3$ Dependent Variable, Independent Predictors and Covariates

$\begin{array}{llllll}1 & 2 & 3 & 4 & 5 & 6\end{array}$

1. W3LTPA ${ }^{\ddagger} 1.00$

2. $\mathrm{W} \mathrm{BI}^{\dagger} \quad .445^{* * *} \quad 1.00$

3. $\mathrm{W} 2 \mathrm{SEb}^{\dagger} \quad .337^{* * *} \quad .738^{* * *} \quad 1.00$

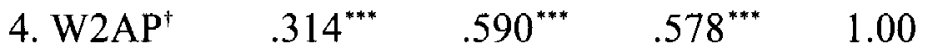

5. W2LTPA W $^{\ddagger} .365^{* * *} \quad .476^{* * *} \quad .428^{* * *} \quad .363^{* * *} \quad 1.00$

6. $\mathrm{INCOME}^{\ddagger} \quad .234^{* *} \quad .257^{* *} \quad .253^{* *} \quad .159 \quad .186^{*} \quad 1.00$

Note. Dependent variable: W3LTPA:Wave 3 Leisure-time Physical Activity; independent predictors: W2BI: Wave 2 Behavioral Intention, W2SEb: Wave 2 Barrier Self-Efficacy; W2AP:Wave 2 Action Planning; covariates: W2LTPA:Wave 2 Leisure-time Physical Activity, INCOME: annual household income. Listwise deletion for all variables yielded $n=131$.

All correlations are Spearman's tho except for intercorrelations between variables 2, 3, and 4, exclusively, which are Pearson's $r$.

${ }^{*} p<.05,{ }^{* *} p<.01,{ }^{* * *} p<.001$.

$\dagger:$ Pearson's $r$

\$: Spearman's rho 
Table 19

H3 Unstandardized Logistic Regression Coefficients, Wald Statistics, Adjusted Odds Ratios, and Confidence Intervals for Model 2 (Full Model)

\begin{tabular}{cccccc}
\hline & $b$ & Wald & $\begin{array}{c}\text { Adjusted } \\
\text { OR }\end{array}$ & $\begin{array}{c}95 \% \text { CI of } \\
\text { OR }\end{array}$ & $p$ \\
\hline Block 2 & & & & & \\
W2BI & .541 & 6.13 & 1.72 & 1.12 to 2.64 & .013 \\
W2SEb & -.003 & .06 & .997 & .97 to 1.02 & $N S$ \\
W2AP & .291 & .94 & 1.34 & .74 to 2.41 & $N S$ \\
Block 1 & & & & & \\
W2LTPA & -.933 & 3.35 & .393 & .15 to 1.07 & $N S$ \\
INCOME & -.611 & 2.13 & .543 & .24 to 1.23 & $N S$ \\
\hline
\end{tabular}

Note. $\mathrm{N}=142$; Dependent variable: binary W3 Leisure-time Physical Activity; Block 2 predictors: W2BI: Wave 2 Behavioral Intention, W2SEb:Wave 2 Barrier Self-Efficacy, W2AP:Wave 2 Action Planning; Block 1 covariates: W2LTPA: Wave 2 Leisure-time Physical Activity, INCOME: annual household income. $b=$ unstandardized logistic regression coefficient, $\mathrm{OR}=$ Odds Ratio, $\mathrm{CI}=$ Confidence Interval, $N S$ $=$ not statistically significant 


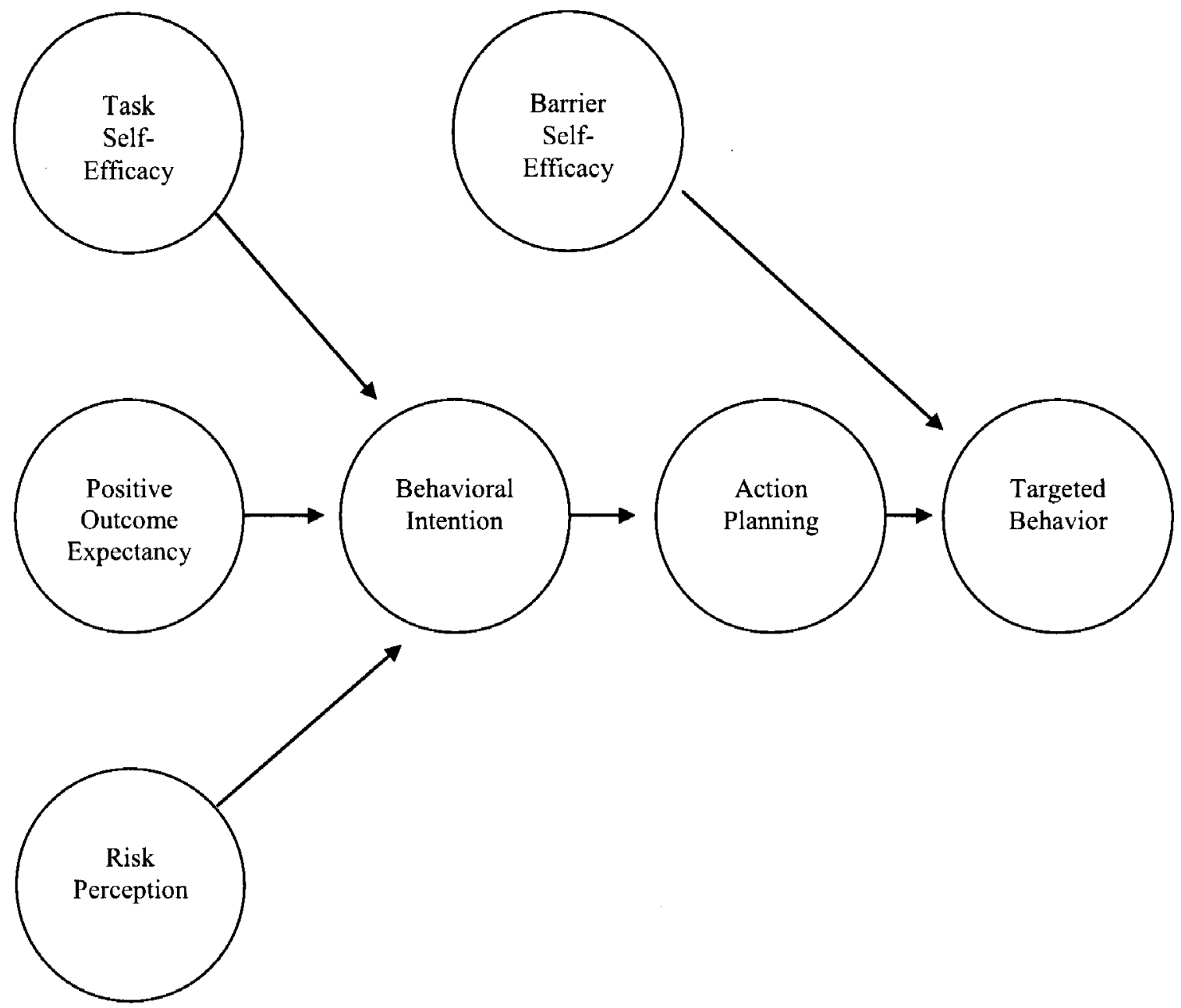

Figure 1. The Health Action Process Approach (basic model depicting behavior initiation). 


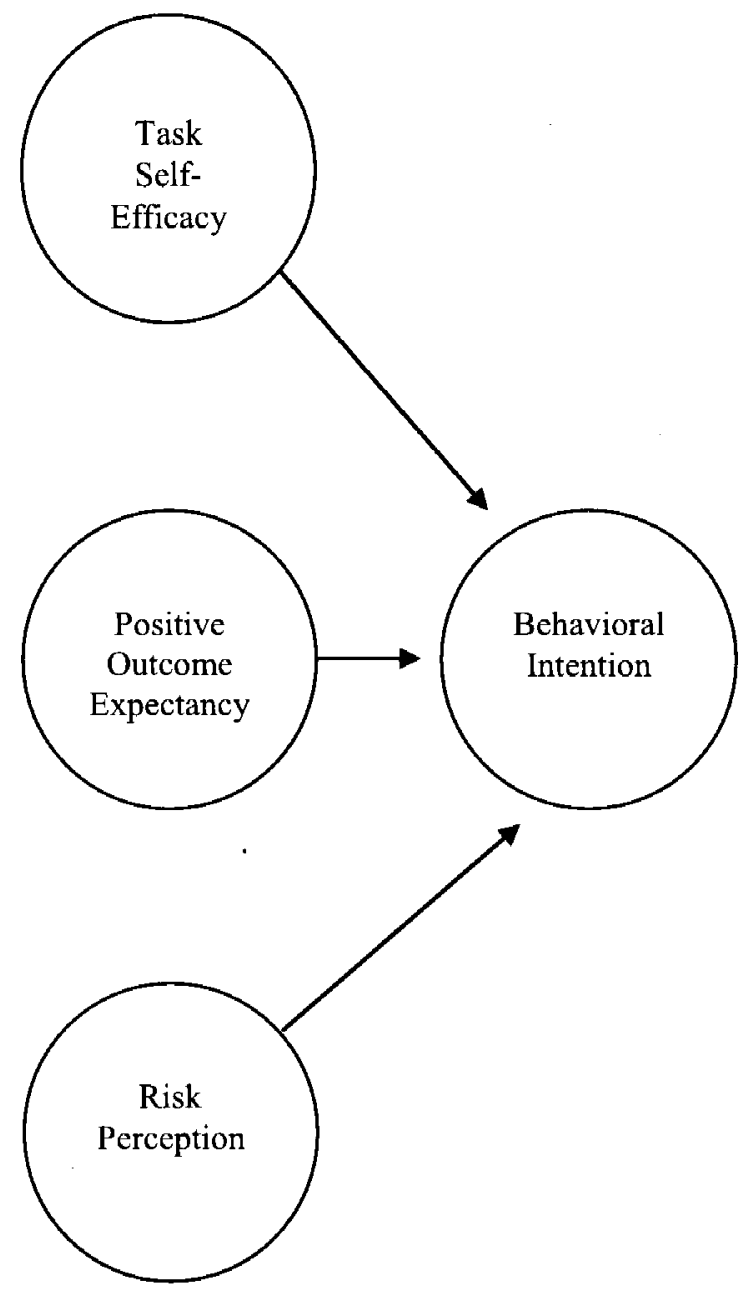

Figure 2. Health Action Process Approach Motivational Stage 


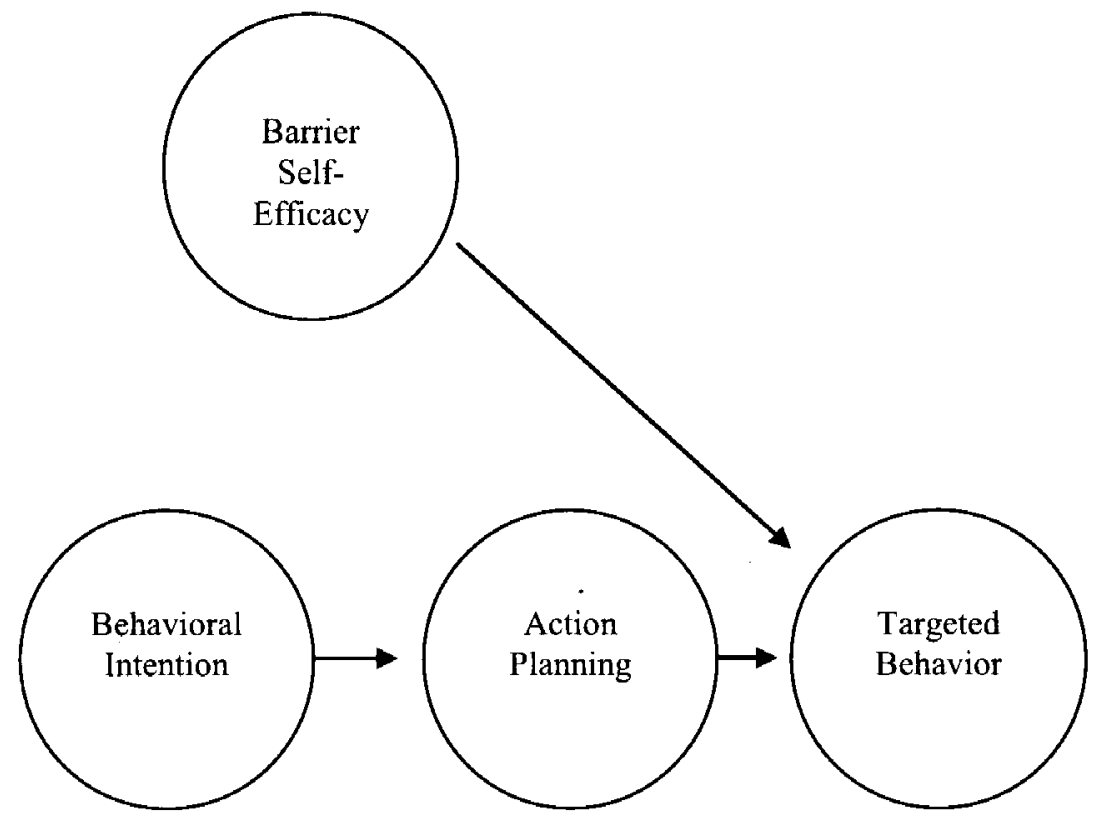

Figure 3. Health Action Process Approach Volitional Stage 


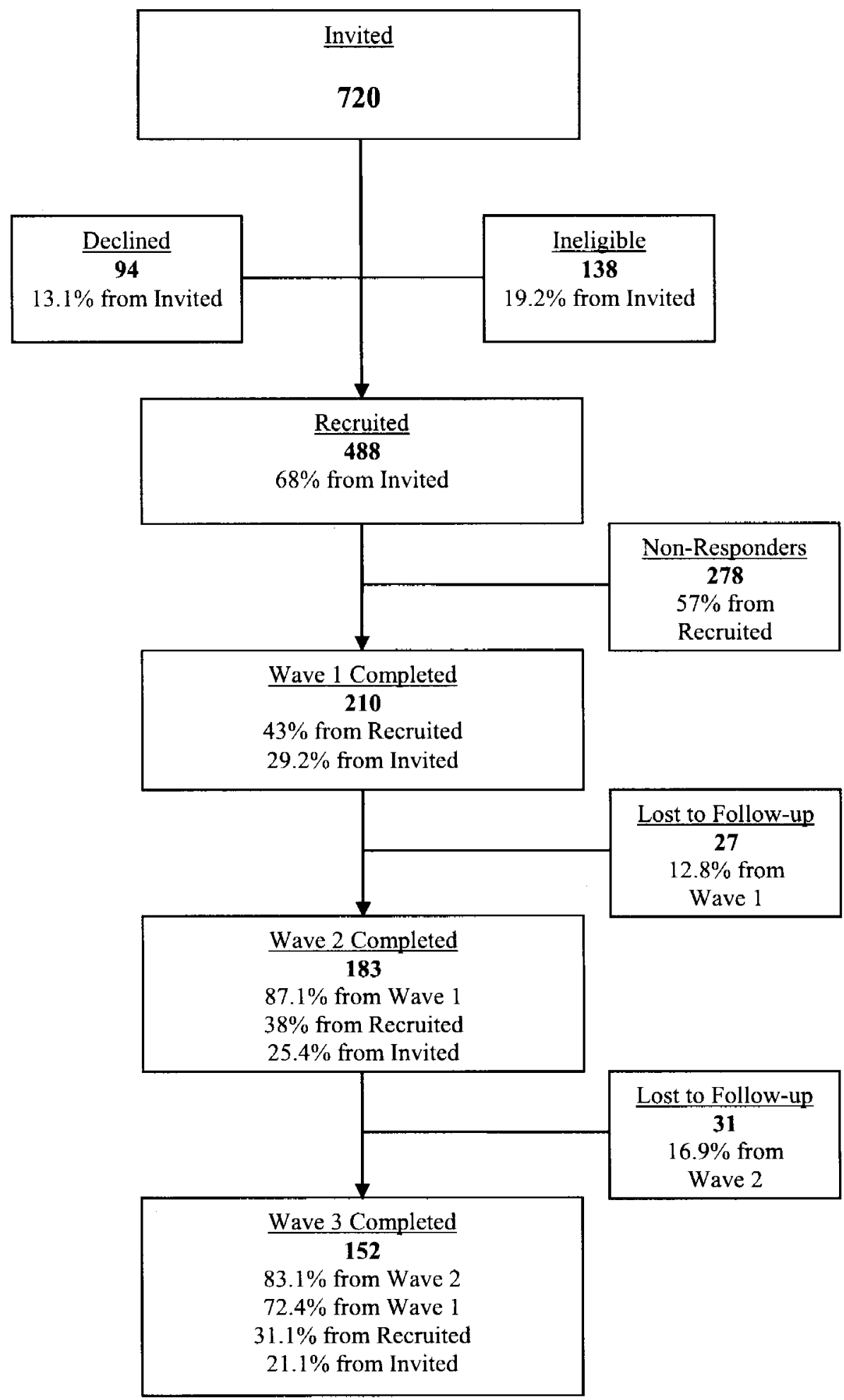

Figure 4. Flow Chart of Study Recruitment (all sites). 


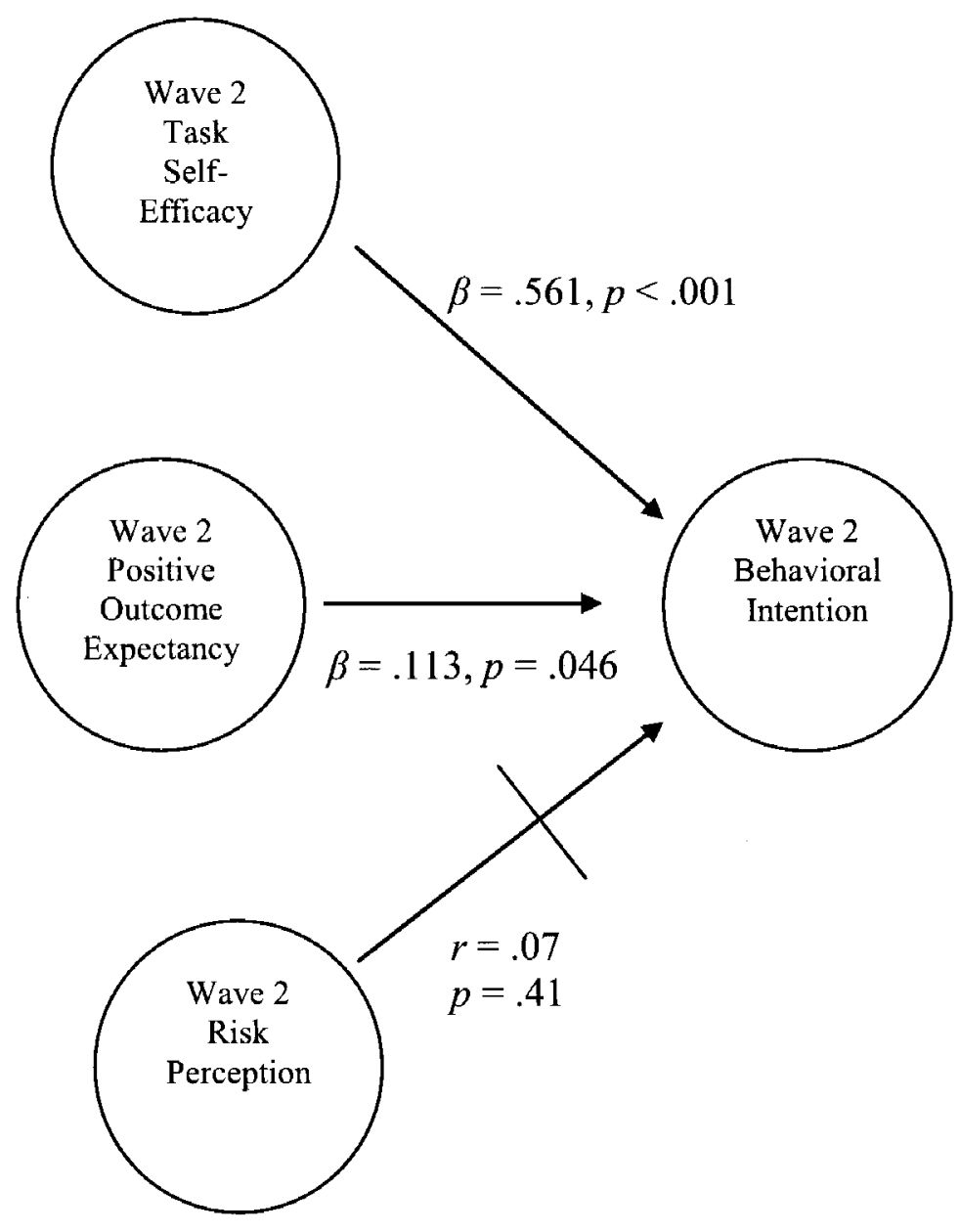

Figure 5. Regression Coefficients of Linear Regression Test of HAPA Motivational Stage Constructs. 


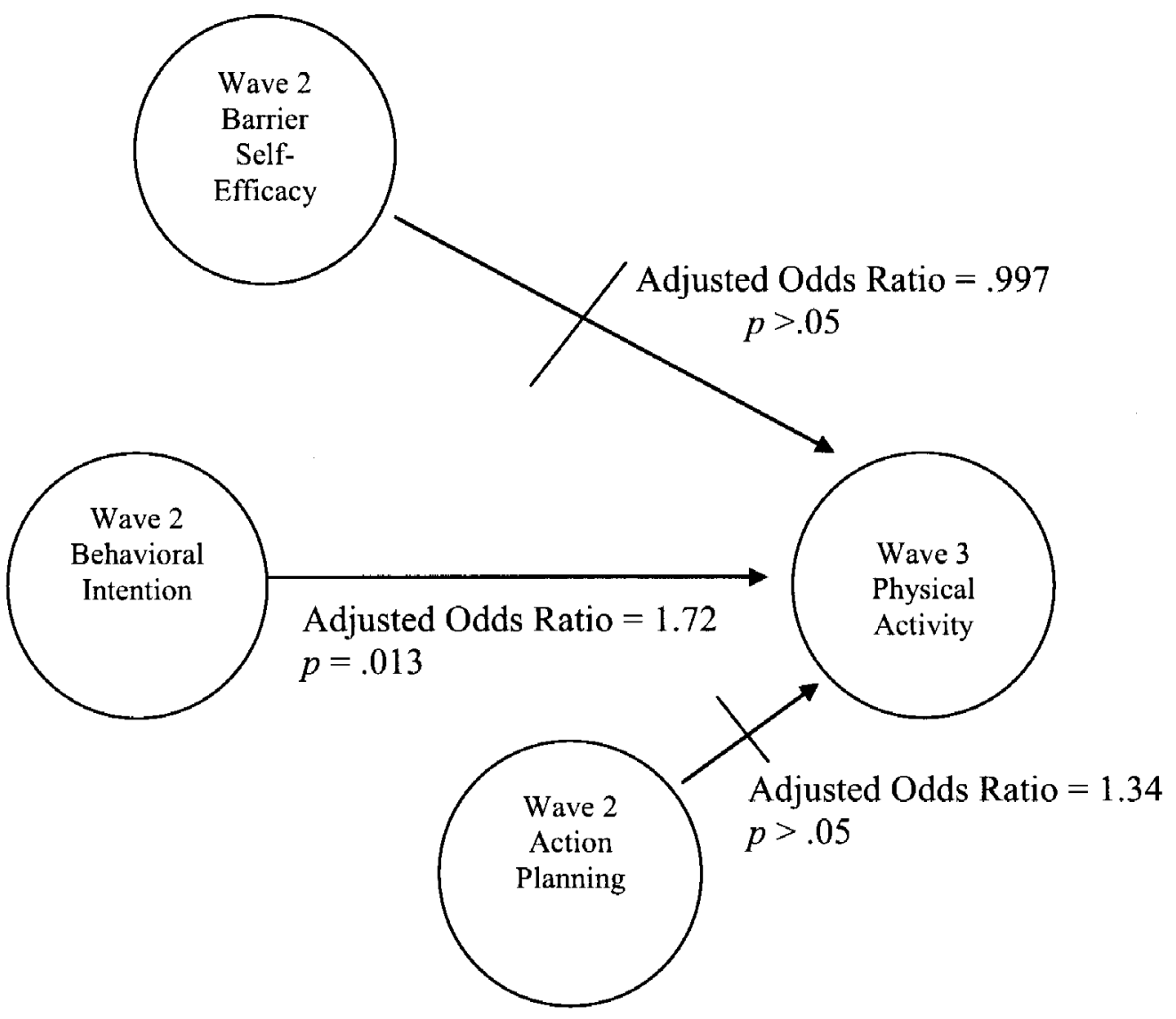

Figure 6. Adjusted Odds Ratios for Logistic Regression Test of HAPA Volitional Stage Constructs. 


\section{REFERENCES}

Absetz, P., Valve, R., Oldenburg, B., Heinonen, H., Nissinen, A., Fogelholm, M.,...Uutela, A. (2007). Type 2 diabetes prevention in the "real world": One-year results of the GOAL implementation trial. Diabetes Care, 30, 2465-2470.

Ajzen, I. (1988). Attitudes, personality, and behavior. Milton Keynes, U.K.: Open University Press.

Ajzen, I. (1991). The theory of planned behavior. Organizational Behavior and Human Decision Processes, 50, 179-211.

Ajzen, I. (2002). Perceived behavioral control, self-efficacy, locus of control, and the theory of planned behavior. Journal of Applied Social Psychology, 32, 665-683.

Albright, A., Franz, M., Hornsby, G., Kriska, A., Marrero, D., Ullrich, I., \& Verity, L. S. (2000). American college of sports medicine position stand: Exercise and type 2 diabetes. Medicine \& Science in Sports \& Psychology, 32, 1345-1360. doi: 10.1249/MSS.0b013e3181949333.

Allen, N. A. (2004). Social cognitive theory in diabetes exercise research: An integrative literature review. The Diabetes Educator, 30, 805-819.

American Association of Diabetes Educators. (2009a). AADE guidelines for the practice of diabetes self-management education and training (DSME/T). The Diabetes Educator, 35, 85S-107S.

American Association of Diabetes Educators. (2009b). AADE-7 self-care behaviors: Being active. Retrieved from http://www.diabeteseducator.org/export/sites/aade/ _resources/pdf/general/AADE7_being_active.pdf

American Diabetes Association. (2002). Diabetes mellitus and exercise. Diabetes Care, 25(Suppl. 1), S64-S68.

American Diabetes Association. (2003). Economic costs of diabetes in the U.S. in 2002. Diabetes Care, 26, 917-932.

American Diabetes Association. (2008). Economic costs of diabetes in the U.S. in 2007. Diabetes Care, 31(3), 1-20. 
American Diabetes Association. (2010). Standards of medical care in diabetes - 2010. Diabetes Care, 33(Suppl. 1), S11-S61.

Andresen, E. M., Malmgren, J. A., Carter, W. B., \& Patrick D.L. (1994). Screening for depression in well older adults: Evaluation of a short form of the CES-D (center for epidemiologic studies depression scale). American Journal of Preventive Medicine, 10, 77-84.

Armitage, C. J., \& Conner, M. (2000). Social cognition models and health behaviour: A structured review. Psychology and Health, 15, 173-189.

Babyak, M. A. (2004). What you see may not be what you get: A brief, nontechnical introduction to overfitting in regression-type models. Psychosomatic Medicine, $66,411-421$.

Bandura, A. (1997). Self-efficacy: The exercise of control. New York, New York: W. H. Freeman and Company.

Baron, R. M., \& Kenny, D. A. (1986). The moderator-mediator variable distinction in social psychological research: Conceptual, strategic, and statistical considerations. Journal of Personality and Social Psychology, 51, 1173-1182.

Bauman, A., Ainsworth, B. E., Bull, F., Craig, C. L., Hagstromer, M., Sallis, J. F.,...Sjostrom, M. (2009). Progress and pitfalls in the use of the international physical activity questionnaire (IPAQ) for adult physical activity surveillance. Journal of Physical Activity and Health, 6(Suppl. 1), S5-S8.

Benjamin, Y., \& Hochberg, Y. (1995). Controlling the false discovery rate: A practical and powerful approach to multiple testing. Journal of the Royal Statistical Society, 57, 289-300.

Blanchard, C. M., Courneya, K. S., Rodgers, W. M., Daub, B., \& Knapik, G. (2002). Determinants of exercise intention and behavior during and after phase 2 cardiac rehabilitation: An application of the theory of planned behavior. Rehabilitation Psychology, 47, 308-323.

Blanchard, C. M., Rodgers, W. M., Courneya, K. S., Daub, B., \& Blonde, L. (2002). Selfefficacy and mood in cardiac rehabilitation: Should gender be considered? Behavioral Medicine, 27, 149-160.

Blonde, L., \& Karter, A. (2005). Current evidence regarding the value of self-monitored blood glucose testing. The American Journal of Medicine, 118(9A), 20S-26S.

Blue, C. L. (2007). Does the theory of planned behavior identify diabetes-related cognitions for intention to be physically active and eat a healthy diet. Public Health Nursing, 24, 141-150. 
Boren, S. A., Fitzner, K. A., Panhalkar, P. S., \& Specker, J. E. (2009). Costs and benefits associated with diabetes education: A review of the literature. The Diabetes Educator, 35, 72-96.

Brewer, N. T., Chapman, G. B., Gibbons, F. X., Gerrard, M., McCaul, K. D., \& Weinstein, N. D. (2007). Meta-analysis of the relationship between risk perception and health behavior: the example of vaccination. Health Psychology, $26,136-145$.

Brewer, N. T., Weinstein, N. D., Cuite, C. L., \& Herrington, J. E. (2004). Risk perception and their relation to risk behavior. Annals of Behavioral Medicine, 27, 125-130.

Buchner, A., Erdfelder, E., Faul, F., \& Lang, A. (2006). G*Power (Version 3.0.5). Retrieved from http://www.psycho.uni-duesseldorf.de/abteilungen/aap/gpower3/

Calles-Escandon, J., \& Cipolla, M. (2001). Diabetes and endothelial dysfunction: A clinical perspective. Endocrine Reviews, 22, 36-52.

Carnethon, M. R., Biggs, M. L., Barzilay, J. I., Smith, N. L., Vaccarino, V., Bertoni, A. G.,...Siscovick, D. (2007). Longitudinal association between depressive symptoms and incident type 2 diabetes mellitus in older adults: The cardiovascular health study. Archives of Internal Medicine, 167, 802-807.

Center for Research on Women with Disabilities. (2009). Health constructs and measurement. Retrieved from http://www.crowdbcm.net/measures/Measures-IHealthoutcomes.htm

Centers for Disease Control and Prevention. (2007). National diabetes fact sheet: General information and national estimates on diabetes in the United States. U.S. Department of Health and Human Services. Retrieved from http://www.cdc.gov/ diabetes/pubs/pdf/ndfs_2007.pdf

Centers for Disease Control and Prevention. (2008). Behavioral risk factor surveillance system: SMART BRFSS city and county data: national center for chronic disease prevention and health promotion. Retrieved from http://apps.nccd.cdc.gov/ BRFSS-SMART/MMSARiskChart.asp?yr=2008\&MMSA= 51\&cat=DB\&qkey $=1363 \& \operatorname{grp}=0$

Centers for Disease Control and Prevention. (2009). Behavioral risk factor surveillance system survey data: National center for chronic disease prevention and health promotion. Retrieved from http://apps.nccd.cdc.gov/brfss/display.asp?cat= $\mathrm{DB} \& \mathrm{yr}=2009$ \&qkey $=1363 \&$ state $=\mathrm{KY}$

Chipkin, S. R., Klugh, S. A., \& Chasan-Taber, L. (2001). Exercise and diabetes. Cardiology Clinics, 19, 489-505. 
Christensen, A. J. (2002). Patient-by-treatment context interaction in chronic disease: A conceptual framework for the study of patient adherence. Psychosomatic Medicine, 62, 435-443.

Cohen, J. (1992). A power primer. Psychological Bulletin, 112, 155-159.

Cohen, J., Cohen, P., West, S. G., \& Aiken, L. S. (2003). Applied Multiple Regression/ Correlation Analysis for the Behavioral Sciences ( $3^{\text {rd }}$ ed.). London: Lawrence Erlbaum Associates.

Conner, M. (2000). Temporal stability as a moderator of relationships in the theory of planned behavior. British Journal of Social Psychology, 39, 469-493.

Conner, M. (2008). Initiation and maintenance of health behaviors. Applied Psychology: An International Review, 57, 42-50.

Courneya, K. S., \& McAuley, E. (1994). Factors affecting the intention-physical activity relationship: Intention versus expectation and scale correspondence. Research Quarterly for Exercise and Sport, 65(3), 280-285.

Cowie, C. C., Rust, K. F., Byrd-Holt, D. D., Eberhardt, M. S., Flegal, K. M., Engelgau, M. M.,...Gregg, E. W. (2006). Prevalence of diabetes and impaired fasting glucose in adults in the U.S. population: National health and nutrition examination survey 1999-2002. Diabetes Care, 29, 1263-1268.

Craig, C. L., Marshall, A. L., Sjorstrom, M., Bauman, A. E., Booth, M. L., Ainsworth, B. E.,...Oja, P. (2003). International physical activity questionnaire: 12-country reliability and validity. Medicine \& Science in Sports \& Psychology, 35, 13811395.

Davies, M. J., Heller, S., Skinner, T. C., Campbell, M. J., Carey, M. E., Cradock, S.,...Khunti, K. (2008). Effectiveness of the diabetes education and selfmanagement for ongoing and newly diagnosed (DESMOND) programme for people with newly diagnosed type 2 diabetes: Cluster randomised controlled trial. British Medical Journal, 336, 491-495.

Desmond, D. W., Tatemichi, T. K., \& Hanzawa, L. (1994). The telephone interview for cognitive status (TICS): Reliability and validity in a stroke sample. International Journal of Geriatric Psychiatry, 9(10), 803-807.

Diabetes Control and Complications Trial Research Group. (1993). The effect of intensive treatment of diabetes on the development and progression of long-term complications in insulin-dependent diabetes mellitus. New England Journal of Medicine, 329, 977-986. 
Diabetes Prevention Program Research Group. (2002). Reduction in the incidence of type 2 diabetes with lifestyle intervention or metformin. New England Journal of Medicine, 346, 393-403.

Diedrich, A., Munroe, D. J., \& Romano, M. (2010). Promoting physical activity for persons with diabetes. The Diabetes Educator, 36, 132-140.

Division of Cancer Control and Population Sciences. (2008). Health behavior constructs: Theory, measurement \& research. Retrieved from http://cancercontrol.cancer.gov/ $\mathrm{brp} /$ constructs /perceived_severity/ps3.html

Donnelly, J. E., Blair, S. N., Jakicic, J. M., Manore, M. M., Rankin, J. W., \& Smith, B. K. (2009). Appropriate physical activity intervention strategies for weight loss and prevention of weight regain for adults: American college of sports medicine position statement. Medicine \& Science in Sports \& Exercise, 41, 459-471.

Dunlap, W. P., Cortina, J. M., Vaslow, J. B., \& Burke, M. J. (1996). Meta-analysis of experiments with matched groups or repeated measures designs. Psychological Methods, 1, 170-177.

Estabrooks, P., Lee, R. E., \& Gyurcsik, N. C. (2003). Resources for physical activity participation: Does availability and accessibility differ by neighborhood socioeconomic status? Annals of Behavioral Medicine, 25, 100-104.

Fillipas, S., Cicuttini, F., Holland, A. E., \& Cherry, C. L. (2010). The international physical activity questionnaire overestimates moderate and vigorous activity in HIV-infected individuals compared with accelerometry. Journal of the Association of Nurses in AIDS Care, 21, 173-181.

Fisher, L., Skaff, M. M., Mullan, J. T., Arean, P., Mohr, D., Masharani, U.,...Laurencin, G. (2007). Clinical depression versus distress among patients with type 2 diabetes: Not just a question of semantics. Diabetes Care, 30, 542-548.

Floyd, D. L., Prentice-Dunn, S., \& Rogers, R. W. (2000). A meta-analysis of research on protection motivation theory. Journal of Applied Social Psychology, 30, 407-429.

Funnell, M. M., Brown, T. L., Childs, B. P., Haas, L. B., Hosey, G. M., Jensen, B.,...Weiss, M. A. (2010). National standards for diabetes self-management education. Diabetes Care, 33(Suppl. 1), S89-S96.

Garcia, K., \& Mann, T. (2003). From 'I wish' to 'I will': Social-cognitive predictors of behavioral intentions. Journal of Health Psychology, 8, 347-360.

Garson, G. D. (2008a). Correlation. Statnotes: Topics in multivariate analyses. Retrieved from http://www2.chass.ncsu.edu/garson/PA765/correl.htm 
Garson, G. D. (2008b). Student's t-test of difference of means. Statnotes: Topics in multivariate analyses. Retrieved from http://www2.chass.ncsu.edu/garson/ PA765/ttest.htm

Garson, G. D. (2009). Generalized linear models and generalized estimating equations. Statnotes: Topics in multivariate analyses. Retrieved from http://faculty.chass. ncsu.edu/garson/PA765/gzlm_gee.htm

Garson, G. D. (2010a). Logistic regression. Statnotes: Topics in multivariate analyses. Retrieved from http:/faculty.chass.ncsu.edu/ garson/PA765/logistic.htm

Garson, G. D. (2010b). Multiple regression. Statnotes: Topics in multivariate analyses. Retrieved from http://faculty.chass.ncsu.edu/garson/PA765/regress.htm\#variables

Gerich, J. E. (2005). The importance of tight glycemic control. The American Journal of Medicine, $118(9 \mathrm{~A}), 7 \mathrm{~S}-11 \mathrm{~S}$.

Glanz, K., Rimer, B. K., \& Lewis, F. M. (2002). Health Behavior and Health Education Theory, Research, and Practice. San Francisco: Wiley \& Sons.

Gollwitzer, P. M. (1993). Goal achievement: The role of intentions. In W. Stroebe \& M. Hewstone (Eds.), European Review of Social Psychology (pp. 141-185) John Wiley \& Sons Ltd.

Greer, T., Dunlap, W. P., Hunter, S. T., \& Berman, M. E. (2006). Skew and internal consistency. The Journal of Applied Psychology, 91, 1351-1358.

Gucciardi, E., Wang, S. C.-T., DeMelo, M., Amaral, L., \& Stewart, D. E. (2008). Characteristics of men and women with diabetes. Canadian Family Physician, 54, 219-227.

Hankonen, N., Absetz, P., Ghisletta, P., Renner, B., \& Uutela, A. (2010). Gender differences in social cognitive determinants of exercise adoption. Psychology \& Health, 25, 55-69.

Harrison, T., \& Stuifbergen, A. (2001). Disability, social support, and concern for children: Depression in mothers with multiple sclerosis. Journal of Obstetric, Gynecologic, and Neonatal Nursing, 31, 444-453.

Herr, N. R. (2010). Mediation with dichotomous outcomes. Retrieved from http://nrherr.bol.ucla. edu/Mediation/logmed.html

International Diabetes Federation. (2010). The MDGs at 10 and civil society. Retrieved from http://www.un-ngls.org/spip.php?page=amdg10\&id_article $=2490$ 
International Monetary Fund. (2009). World economic outlook: Crisis and recovery. Retrieved from http://www.imf.org/external/pubs/ft/weo/2009/01/pdf/text.pdf

Irwin, M., Artin, K. H., \& Oxman, M. N. (1999). Screening for depression in the older adult: Criterion validity of the 10 -item center for epidemiological studies depression scale (CES-D). Archives of Internal Medicine, 159, 1701-1704.

Jack, Jr. L. (2003). Diabetes self-management education research: An international review of intervention methods, theories, community partnerships and outcomes. Diabetes Management \& Health Outcomes, 11, 415-428.

Jerant, A., Kravitz, R., Moore-Hill, M., \& Franks, P. (2008). Depressive symptoms moderated the effect of chronic illness self-management training on self-efficacy. Medical Care, 46, 523-531.

Johnson-Kozlow, M., Sallis, J. F., Gilpin, E. A., Rock, C. L., \& Pierce, J. P. (2006). Comparative validation of the IPAQ and the 7-day PAR among women diagnosed with breast cancer. International Journal of Behavioral Nutrition and Physical Activity, 3(7), 1-10.

King, D. K., \& Glasgow, R. E. (2003). Behavioral self-management of type 2 diabetes: Key issues, evidence-based recommendations, and future directions. In J.A.Trafton \& W.P.Gordon (Eds.), Best practices in the behavioral management of chronic disease (Vol. 2). Los Altos, CA: Institute for Disease Management.

Kirk, A., Mutrie, N., MacIntyre, P., \& Fisher, M. (2003). Increasing physical activity in people with type 2 diabetes. Diabetes Care, 26(4), 1186-1192. Retrieved from $\mathrm{http}: / /$ care. diabetesjournals.org/cgi/content/abstract/26/4/1186

Kline, R. B. (2005). Principles and practice of structural equation modeling. ( $2^{\text {nd }}$ ed.). New York: The Guilford Press.

Kozakova, M., Palombo, C., Mhamdi, L., Konrad, T., Nilsson, P., Staehr, P. B.,...Balkau, B. (2007). Habitual physical activity and vascular aging in a young to middle-age population at low cardiovascular risk. Stroke, 38, 2549-2555.

Krein, S. L., Heisler, M., Piette, J. D., Makki, F., \& Kerr, E. A. (2005). The effect of chronic pain on diabetes patients' self-management. Diabetes Care, 28(1), 65-70.

Krishnan, S., Rosenberg, L., Djousse, L., Cupples, L. A., \& Palmer, J. R. (2007). Overall and central obesity and risk of type 2 diabetes in U.S. black women. Obesity, 15 , $1860-1866$. 
Laatikainen, T., Dunbar, J. A., Chapman, A., Kilkkinen, A., Vartiainen, E., Heistaro, S.,...Janus, E. D. (2007). Prevention of type 2 diabetes by lifestyle intervention in an Australian primary health care setting: Greater green triangle (GGT) diabetes prevention project. Bio-Med Central Public Health, 7, 249-255.

Lewis, K. S., \& Bradley, C. (1994). Measures of Diabetes-Specific Health Beliefs. In C. Bradley (Ed.), Handbook of Psychology and Diabetes (pp. 247-289). Amsterdam, The Netherlands: Harwood Academic Publishers.

Lewis, K. S., Jennings, A. M., Ward, J. D., \& Bradley, C. (1990). Health belief scales developed specifically for people with tablet-treated type 2 diabetes. Diabetic Medicine, 7, 148-155.

Lin, E. H. B., Katon, W., Korff, M. V., Rutter, C., Simon, G. E., Oliver, M.,...Young, B. (2004). Relationship of depression and diabetes self-care, medication adherence, and preventive care. Diabetes Care, 27, 2154-2160.

Lindstrom, J., Louheranta, A., Mannelin, M., Rastas, M., Salminen, V., Eriksson, J.,...Tuomilehto, J. (2003). The Finnish diabetes prevention study (DPS): Lifestyle intervention and 3-year results on diet and physical activity. Diabetes Care, 26, 3230-3236.

Lippke, S., Wiedemann, A. U., Ziegelmann, J. P., Reuter, T., \& Schwarzer, R. (2009). Self-efficacy moderates the mediation of intentions into behavior via plans. American Journal of Health Behavior, 33, 521-529.

Lippke, S., \& Ziegelmann, J. P. (2008). Theory-based health behavior change: Developing, testing, and applying theories for evidence-based interventions. Applied Psychology: An International Review, 57, 698-716.

Lippke, S., Ziegelmann, J. P., \& Schwarzer, R. (2005). Stage-specific adoption and maintenance of physical activity: Testing a three-stage model. Psychology of Sport and Exercise, 6, 585-603.

Lustman, P., Penckofer, S. M., \& Clouse, R. E. (2007). Recent advances in understanding depression in adults with diabetes. Current Diabetes Reports, 7, 114-122.

Maciejewski, P. K., Prigerson, H. G., \& Mazure, C. M. (2000). Self-efficacy as a mediator between stressful life events and depressive symptoms: Differences based on a history of prior depression. The British Journal of Psychiatry, 176, 373-378.

MacKinnon, D. P., \& Dwyer, J. H. (1993). Estimating mediated effects in prevention studies. Evaluation Review, 17, 144-158. 
Maddison, R., \& Prapavessis, H. (2004). Using self-efficacy and intention to predict exercise compliance among patients with ischemic heart disease. Journal of Sport \& Exercise Psychology, 26, 511-524.

Maddux, J. E. (1993). Social cognitive models of health and exercise behavior: An introduction and review of conceptual issues. Journal of Applied Sport Psychology, 5, 116-140.

Marcus, B. H., Dubbert, P. M., Forsyth, L. H., McKenzie, T. L., Stone, E. J., Dunn, A. L.,...Blair, S. N. (2000). Physical activity behavior change: Issues in adoption and maintenance. Health Psychology, 19(Suppl. 1), 32-41.

Marlatt, G. A., Baer, J. S., \& Quigley, L. A. (1995). Self-efficacy and addictive behavior. In A. Bandura (Ed.), Self-efficacy in changing societies (pp. 289-315). New York: Cambridge University Press.

Marshall, S. J., \& Biddle, S. J. H. (2001). The transtheoretical model of behavior change: A meta-analysis of applications to physical activity and exercise. Annals of Behavioral Medicine, 23, 229-246.

McAuley, E., \& Mihalko, S. L. (1998). Measuring Exercise-Related Self-Efficacy. In J.L. Duda (Ed.), Advances in sport and exercise psychology measurement (pp. 371390). Morgantown, W.V.: Fitness Information Technology, Inc.

McClelland, G. H., \& Judd, C. M. (1993). Statistical difficulties of detecting interactions and moderator effects. Psychological Bulletin, 114, 376-390.

McNabb, W. L. (1997). Adherence in diabetes: Can we define it and can we measure it? Diabetes Care, 20, 215-218.

Michigan Diabetes Research and Training Center. (1998). Diabetes History. Retrieved from http://www.med.umich.edu/mdrtc/profs/survey.html\#dmh

Mulcahy, K., Maryniuk, M., Peeples, M., Peyrot, M., Tomky, D., Weaver, T.,...Y Yarborough, P. (2003). Diabetes self-management education core outcomes measures. The Diabetes Educator, 29, 768-803.

Norris, S. L., Engelgau, M. M., \& Venkat-Narayan, K. M. (2001). Effectiveness of selfmanagement training in type 2 diabetes: A systematic review of randomized controlled trials. Diabetes Care, 24, 561-587.

Omondi, D. O., Walingo, M. K., Mbagaya, G. M., \& Othuon, L. O. A. (2010). Understanding physical activity behavior of type 2 diabetics using the theory of planned behavior and structural equation modeling. International Journal of Social Sciences, 5, 160-167. 
Pedersen, B. K., \& Saltin, B. (2006). Evidence for prescribing exercise as therapy in chronic disease. Scandinavian Journal of Medicine \& Science in Sports, 16(Suppl. 1), 3-63.

Peduzzi, P. N., Concato, J., Holford, T. R., \& Feinstein, A. R. (1995). The importance of events per variable in multivariate analysis: Accuracy and precision of regression estimates. Journal of Clinical Epidemiology, 48, 1503-1510.

Peduzzi, P. N., Concato, J., Kemper, E., Holford, T. R., \& Feinstein, A. R. (1996). A simulation study of the number of events per variable in logistic regression analysis. Journal of Clinical Epidemiology, 49, 1373-1379.

Plotnikoff, R. C., Lippke, S., Courneya, K. S., Birkett, N., \& Sigal, R. (2010). Physical activity and diabetes: An application of the theory of planned behavior to explain physical activity for Type 1 and Type 2 diabetes in an adult population sample. Psychology and Health, 25, 7-23.

Preacher, A. K. (2009). SPSS SOBEL Macro Syntax Reference. Retrieved from http://www. comm.ohio-state.edu/ahayes/SPSS\% 20programs/ Sobel\%20syntax .pdf

Preacher, A. K. (2010). Reporting bootstrap results. Retrieved from http://www. facebook.com/topic.php?uid=44574520333\&topic $=7795$

Preacher, K. J., \& Hayes, A. F. (2004). SPSS and SAS procedures for estimating indirect effects in simple mediation models. Behavior Research Methods, Instruments, \& Computers, 36, 717-731.

Preacher, K. J., Rucker, D. D., \& Hayes, A. F. (2007). Addressing moderated mediation hypothesis: Theory, methods, and prescriptions. Multivariate Behavioral Research, 42, 185-227.

Prince, S. A., Adamo, K. B., Hamel, M. E., Hardt, J., Gorber, S. C., \& Tremblay, M. (2008). A comparison of direct versus self-report measures for assessing physical activity in adults: A systematic review. International Journal of Behavioral Nutrition and Physical Activity. Retrieved from http://www.ijbnpa.org/content/ $5 / 1 / 56$

Prochaska, J. O., \& DiClemente, C. C. (1984). The transtheoretical approach: Crossing the traditional boundaries of change. Homewood, IL: J. Irwin.

Renner, B., \& Schwarzer, R. (2003). Social-cognitive factors in health behavior change. In Jerry Suls \& Kenneth A.Wallston (Eds.), Social psychological foundations of health and illness (pp. 169-196). Oxford: Blackwell Publishing Ltd. 
Reuter, T., Ziegelmann, J. P., Lippke, S., \& Schwarzer, R. (2009). Long-term relations between intentions, planning, and exercise. A 3-year longitudinal study after orthopedic rehabilitation. Rehabilitation Psychology, 54, 363-371.

Rizvi, A. A. (2004). Type 2 diabetes: Epidemiologic trends, evolving pathogenic concepts, and recent changes in therapeutic approach. Southern Medical Journal, 97, 1079-1087.

Rogers, R. W. (1983). Cognitive and physiological processes in fear appraisals and attitude change: A revised theory of protection motivation. In J.T. Cacioppo \& R. E. Petty (Eds.), Social psychophysiology (pp. 153-176). New York: Guilford Press.

Rosenstock, I. M. (1974). Historical origins of the health belief model. Health Education Monographs, 2, 1-8.

Sallis, J. F., \& Saelens, B. E. (2000). Assessment of physical activity by self-report: Status, limitations, and future directions. Research Quarterly for Exercise and Sport, 71, 1-14.

Scholz, U., Schuz, B., Ziegelmann, J. P., Lippke, S., \& Schwarzer, R. (2008). Beyond behavioral intentions: Planning mediates between intentions and physical activity. British Journal of Health Psychology, 13, 479-494.

Scholz, U., Sniehotta, F. F., \& Schwarzer, R. (2005). Predicting physical exercise in cardiac rehabilitation: The role of phase-specific self-efficacy beliefs. Journal of Sport \& Exercise Psychology, 27, 135-151.

Schwarzer, R. (1992). Self-efficacy in the adoption and maintenance of health behaviors: Theoretical approaches and a new model. In R. Schwarzer (Ed.), Self-efficacy: Thought control of action. London, England: Hemisphere Publishing Corporation.

Schwarzer, R. (1999). Self-regulatory processes in the adoption and maintenance of health behaviors: The role of optimism, goals, and threats. Journal of Health Psychology, 4, 115-127.

Schwarzer, R. (2008). Modeling health behavior change: How to predict and modify the adoption and maintenance of health behaviors. Applied Psychology: An International Review, 57, 1-29.

Schwarzer, R., Luszczynska, A., Ziegelmann, J. P., Scholz, U., \& Lippke, S. (2008). Social-cognitive predictors of physical exercise adherence: Three longitudinal studies in rehabilitation. Health Psychology, 27(Suppl.), S54-S63.

Schwarzer, R., \& Renner, B. (2000). Social-cognitive predictors of health behavior: Action self-efficacy and coping self-efficacy. Health Psychology, 19, 487-495. 
Schwarzer, R., Schuz, B., Ziegelmann, J. P., Lippke, S., Luszczynska, A., \& Scholz, U. (2007). Adoption and maintenance of four health behaviors: Theory-guided longitudinal studies on dental flossing, seat belt use, dietary behavior, and physical activity. Annals of Behavioral Medicine, 33(2), 156-166.

Schwarzer, R., Sniehotta, F. F., Lippke, S., Luszczynska, A., Scholz, U., Schuz, B.,...Ziegelmann, J. P. (2003). On the assessment and analysis of variables in the health action process approach: Conducting an investigation. Retrieved from http://web.fu-berlin.de/gesund/hapa_web.pdf

Shaw, J. E., Sicree, R. A., \& Zimmet, P. Z. (2010). Global estimates of the prevalence of diabetes for 2010 and 2030. Diabetes Research and Clinical Practice, 87, 4-14.

Sherwood, N. E., \& Jeffery, R. W. (2000). The behavioral determinants of exercise: Implications for physical activity interventions. Annual Review of Nutrition, 20, 21-44.

Sjostrom, M., Ainsworth, B. E., Bauman, A. E., Bull, F., Craig, C., \& Sallis, J. F. (2005). Guidelines for data processing and analysis of the international physical activity questionnaire (IPAQ). Retrieved from http://www.ipaq.ki.se/scoring.htm

Skelly, A. H., Marshall, J. R., Haughey, B. P., Davis, P. J., \& Dunford, R. G. (1995). Self-efficacy and confidence in outcomes as determinants of self-care practices in inner-city, african-american women with non-insulin-dependent diabetes. The Diabetes Educator, 2l(1), 38-46.

Sniehotta, F. F., Scholz, U., \& Schwarzer, R. (2005). Bridging the intention-behaviour gap: Planning, self-efficacy, and action control in the adoption and maintenance of physical exercise. Psychology \& Health, 20, 143-160.

Sniehotta, F. F., Scholz, U., \& Schwarzer, R. (2006). Action plans and coping plans for physical exercise: A longitudinal intervention study in cardiac rehabilitation. British Journal of Health Psychology, 11, 23-37.

Sniehotta, F. F., Schwarzer, R., Scholz, U., \& Schuz, B. (2005). Action planning and coping planning for long-term lifestyle change: Theory and assessment. European Journal of Social Psychology, 35, 565-576.

Sutton, S. (2008). How does the health action process approach (HAPA) bridge the intention-behavior gap? An examination of the model's causal structure. Applied Psychology: An International Review, 57, 66-74.

The Associated Press. (2009). Diabetics skimp on lifesaving care in recession. Retrieved from http://www.nydailynews.com/lifestyle/health/2009/04/13/2009-04-13_ diabetics_cut_back_on_health_care_in_recession.html 
Thomas, N., Alder, E., \& Leese, G. P. (2004). Barriers to physical activity in patients with diabetes. Postgraduate Medical Journal, 80, 287-291.

Tudor-Locke, C. E., \& Myers, A. M. (2001). Challenges and opportunities for measuring physical activity in sedentary adults. Sports Medicine, 31, 91-100.

U.K. Prospective Diabetes Study (UKPDS) Group. (1998). Intensive blood-glucose control with sulphonylureas or insulin compared with conventional treatment and risk of complications in patients with type 2 diabetes (UKPDS 33). The Lancet, $352,837-853$.

U.S. Census Bureau. (2010). State and County Quick Facts. Retrieved from http://quickfacts.census.gov/qfd/states/21000.html

Uutela, A., Absetz, P., Nissinen, A., Valve, R., Talja, M., \& Fogelholm, M. (2004). Health psychological theory in promoting population health in Paijat-Hame, Finland: First steps toward a type 2 diabetes prevention study. Journal of Health Psychology, 9(1), 73-84.

Velicer, W. F., \& Prochaska, J. O. (2008). Stage and non-stage theories of behavior and behavior change: A comment on Schwarzer. Applied Psychology: An International Review, 57, 75-83.

Vittinghoff, E., \& McCulloch, C. E. (2007). Relaxing the rule of ten events per variable in logistic and cox regression. American Journal of Epidemiology, 165, 710-718.

Walker, E. A., \& Usher, J. A. (2003). Understanding and enhancing adherence in adults with diabetes. Current Diabetes Reports, 3, 141-148.

Weinstein, N. D. (2000). Perceived probability, perceived severity, and health-protective behavior. Health Psychology, 19, 65-74.

Weinstein, N. D., Rothman, A. J., \& Sutton, S. (1998). Stage theories of health behavior: Conceptual and methodological issues. Health Psychology, 17, 290-299.

Wiedemann, A. U., Schuz, B., Sniehotta, F., Scholz, U., \& Schwarzer, R. (2009). Disentangling the relation between intentions, planning, and behavior: A moderated mediation analysis. Psychology and Health, 24, 67-79.

Wild, S., Roglic, G., Green, A., Sicree, R., \& King, H. (2004). Global prevalence of diabetes: estimates for the year 2000 and projections for 2030. Diabetes Care, 27, 1047-1053.

Williams, D. M., Anderson, E. S., \& Winett, R. A. (2005). A review of the outcome expectancy construct in physical activity research. Annals of Behavioral Medicine, 29, 70-79. 
Williams, D. M., Lewis, B. A., Dunsiger, S., Whiteley, J. A., Papandonatos, G. D., Napolitano, M. A.,... Marcus, B. H. (2008). Comparing psychosocial predictors of physical activity adoption and maintenance. Annals of Behavioral Medicine, 36, 186-194.

Williams, K. E., \& Bond, M. J. (2002). The roles of self-efficacy, outcome expectancies, and social support in the self-care behaviors of diabetics. Psychology, Health, \& Medicine, 7(2), 127-141.

Wing, R. R., Sallis, J. F., Goldstein, M. G., Smith-West, D., Acton, K. J., Jeffery, R. W.,...Jakicic, J. M. (2001). Behavioral science research in diabetes: Lifestyle changes related to obesity, eating behavior, and physical activity. Diabetes Care, $24,117-123$.

World Health Organization. (2010). Global database on body mass index. Retrieved from http://apps.who.int/bmi/index.jsp?introPage=intro_3.html

Zhang, P., Zhang, X., Brown, J., Vistisen, D., Sicree, R., Shaw, J., \& Nichols, G. (2010). Global healthcare expenditure on diabetes for 2010 and 2030. Diabetes Research and Clinical Practice, 87, 293-301.

Ziegelmann, J. P., Luszczynska, A., Lippke, S., \& Schwarzer, R. (2007). Are goal intentions or implementation intentions better predictors of health behavior? A longitudinal study in orthopedic rehabilitation. Rehabilitation Psychology, 52(1), 97-102.

Zinman, B., Ruderman, N., Campaigne, B. N., Devlin, J. T., \& Schneider, S. H. (2002). American diabetes association (position statement): Diabetes mellitus and exercise. Diabetes Care, 25(Suppl. 1), S64-S68. 


\section{APPENDIX}

\section{Study Measures}

\section{Eligibility Screening:}

\section{Attitudes and Beliefs about Physical Activity and Type II Diabetes}

Directions: Please answer the following questions to determine if you are eligible to participate in this study. Please answer each question by checking the box (YES or NO) that best answers the question for you. After selecting your answers, please review the instructions on Page 2.

1) Are you 21 years old or older?

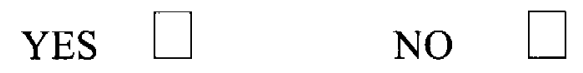

2) Have you been diagnosed by your doctor as having type 2 diabetes (also referred to as adult-onset or non-insulin dependent diabetes)?

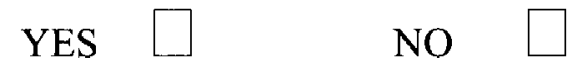

3) Can you read, write, and understand English?

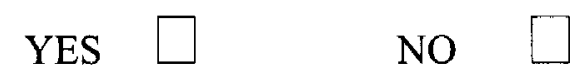

4) Do you have access to a working telephone number?

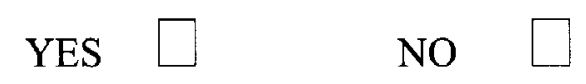

5) Have you been told by your doctor that you cannot participate in moderate or vigorous physical activity?

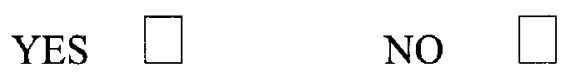


6) Are you able to participate in any physical activity?

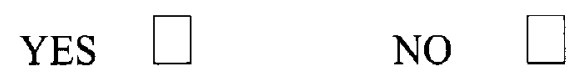

7) Do you currently exercise or participate in physical activity 4 or more days a week for 30 accumulated minutes each day (i.e., 30 or more total minutes each day, 4 days a week)?

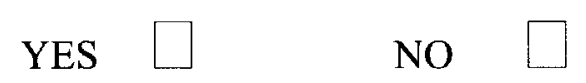

Screening Instructions:

If you answered "NO" for Questions 1 through 4, thank you for your time. Unfortunately, you are currently ineligible to participate in this study. Please return any study materials in this packet to your first Diabetes Education class. Thank you.

If you answered "YES" to either Question 5 or 7, thank you for your time. Unfortunately, you are currently ineligible to participate in this study. Please return any study materials in this packet to your first Diabetes Education class. Thank you.

*If neither of these situations above apply to you, you are ELIGIBLE to participate in this study. Please follow the directions provided on the survey packet to complete the questionnaires and return them to the first Diabetes Education class. You may also choose to mail back your questionnaires in the provided pre-addressed/stamped envelope.

*PLEASE COMPLETE THE FOLLOWING INFORMATION: If you are eligible to participate, we will need your name, mailing address, and telephone number for two reasons. First, to send you compensation for your time in the form of a Kroger Gift Card for each wave or time you participate in the study. Second, the third wave or time of this study is telephone-based. This means that the study coordinator or a member of the study team will contact you at the telephone number you provide to ask you the last set of questions to complete this study, approximately 6-weeks after you begin this study.

NAME:

MAILING ADDRESS:

BEST TELEPHONE NUMBER TO REACH YOU: 
Demographics

\section{GENERAL BACKGROUND INFORMATION}

1. Today's date: (month/day/year)

2. How old are you? (years old)

3. Gender

$\square \quad$ Female $\quad \square \quad$ Male

4. How tall are you?

_ feet __ inches

5. How much do you currently weigh?

_ _ _ pounds

6. Ethnic group (circle one):

1. White (non-Hispanic)

2. Black

3. Hispanic

4. Asian

5. Specify

7. Marital status (circle one):

1. Never married

2. Currently married

3. Separated

4. Divorced

5. Widowed

8. Current living arrangement (circle one):

1. Live alone

2. Live $w /$ spouse/partner

3. Live $w /$ spouse/partner and children

4. Live with children (no spouse/partner)

5. Live with roommate who is not partner

6. Live with parents

7. Other (specify) 
9. Level of school completed (circle one):

1. Less than $7^{\text {th }}$ grade

2. Junior high school $\left(7^{\text {th }}, 8^{\text {th }}, \& 9^{\text {th }}\right.$ grade $)$

3. Partial high school $\left(10^{\text {th }}\right.$ or $11^{\text {th }}$ grade $)$

4. High School graduate (included G.E.D.)

5. Partial college or training

6. College or university

7. Graduate professional (graduate degree)

10. Approximate annual gross income for your household: (circle one):

(Remember all information you provide will remain completely confidential)

1. Less than $\$ 10,000$

2. $\$ 10,000-\$ 19,999$

3. $\$ 20,000-\$ 39,999$

4. $\$ 40,000-\$ 59,999$

5. $\$ 60,000-\$ 100,000$

6. Greater than $\$ 100,000$

11. Which category best describes your usual occupation? If you are not currently employed, which category best describes your LAST job? (circle one number):

1. Professional (e.g., teachers/professors, nurses, lawyers, physicians, \& engineers)

2. Manager/Administrator (e.g., sales managers)

3. Clerical (e.g., secretaries, clerks or mail carriers)

4. Sales (e.g., sales persons, agents \& brokers)

5. Service (e.g., police, cooks, waitress, or hairdressers)

6. Skilled Crafts, Repairer (e.g., carpenters)

7. Equipment or Vehicle Operator (e.g., truck drivers)

8. Laborer (e.g., maintenance factory workers)

9. Farmer (e.g., owners, managers, operators or tenants)

10. Member of the military

11. Homemaker (with no job outside the home)

12. Other (please describe)

12. Current employment situation (circle all that apply):

1. Full time at job

2. Part time at job

3. On leave with pay

4. On leave without pay

5. Disabled

6. Seeking work

7. Retired

8. Homemaker

9. Student 


\section{YOUR DIABETES HISTORY}

We would like to ask you about the health care you have received recently. Please answer every question by filling in the blank(s), circling the correct answer, or checking the correct box.

Q1. How long have you been diagnosed with type 2 diabetes? years months

Q2. Do you currently take insulin to treat your diabetes? yes no

Q3. Are you currently taking medications for high cholesterol? yes no

Q4. Have you ever been told by a health care provider that you have any of the following problems related to your heart or circulation? (circle one answer on each line)

\begin{tabular}{|llcc|}
\hline & & No & Yes \\
\hline A. & Heart attack & 1 & 2 \\
\hline B. & Heart failure & 1 & 2 \\
\hline C. & High cholesterol & 1 & 2 \\
\hline D. & Angina & 1 & 2 \\
\hline
\end{tabular}

Q5. Have you ever been told by a health care provider that you have high blood pressure? (check one box)

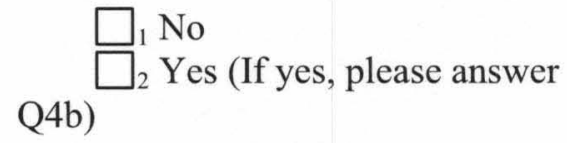

Q5b. Do you now take medication for your high blood pressure? (Check one box)

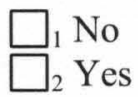

Q6. Have you ever had any of the following operations or procedures related to your heart? (circle one answer on each line)

\begin{tabular}{|llcc|}
\hline & & No & Yes \\
\hline A. & Coronary artery bypass surgery (open heart surgery) & 1 & 2 \\
\hline B. & Coronary angioplasty ("balloon" heart procedure) & 1 & 2 \\
\hline C. & Heart catheterization (angiogram) & 1 & 2 \\
\hline
\end{tabular}


Q7. Have you ever been told by a health care provider that you have any of the following problems with your feet or legs? (circle one answer on each line)

\begin{tabular}{|llcc|}
\hline & & No & Yes \\
\hline A. & Peripheral vascular disease (poor circulation in the legs) & 1 & 2 \\
\hline B. & Intermittent claudication (cramping in the calves after exercise) & 1 & 2 \\
\hline C. & $\begin{array}{l}\text { Peripheral neuropathy (nerve problems causing numbness, } \\
\text { tingling, or burning) }\end{array}$ & 1 & 2 \\
\hline
\end{tabular}

Q8. Have you ever been told by a health care provider that you have had any of the following problems? (circle one answer on each line)

\begin{tabular}{|llcc|}
\hline & & No & Yes \\
\hline A. & Stroke & 1 & 2 \\
\hline B. & Transient ischemic attacks (TIA or "mini-strokes") & 1 & 2 \\
\hline
\end{tabular}




\section{Mood}

Below is a list of some of the ways you may have felt or behaved. Please indicate how often you have felt this way during the PAST WEEK by checking the appropriate box for each question.

\begin{tabular}{|c|c|c|c|c|}
\hline & $\begin{array}{l}\text { Rarely or } \\
\text { none of the } \\
\text { time } \\
\text { (less than } 1 \\
\text { day) }\end{array}$ & $\begin{array}{l}\text { Some or a } \\
\text { little of the } \\
\text { time } \\
(1-2 \text { days })\end{array}$ & $\begin{array}{l}\text { Occasionally } \\
\text { or a moderate } \\
\text { amount of } \\
\text { time } \\
(3-4 \text { days })\end{array}$ & $\begin{array}{c}\text { All of } \\
\text { the time } \\
(5-7 \\
\text { days })\end{array}$ \\
\hline \multicolumn{5}{|c|}{$\begin{array}{l}\text { Was bothered by things that usually don't } \\
\text { bother me }\end{array}$} \\
\hline \multicolumn{5}{|c|}{$\begin{array}{l}\text { I had trouble keeping my mind on what I was } \\
\text { doing }\end{array}$} \\
\hline \multicolumn{5}{|l|}{ I felt depressed } \\
\hline \multicolumn{5}{|c|}{ I felt that everything I did was an effort } \\
\hline \multicolumn{5}{|c|}{ I felt hopeful about the future } \\
\hline \multicolumn{5}{|l|}{ I felt fearful } \\
\hline \multicolumn{5}{|l|}{ My sleep was restless } \\
\hline \multicolumn{5}{|l|}{ I was happy } \\
\hline \multicolumn{5}{|l|}{ I felt lonely } \\
\hline I could not "get going" & & & & \\
\hline
\end{tabular}




\section{Diabetes Health Beliefs}

(Severity)

In this section would you please circle a number on each of the scales to indicate how serious you think the following problems would be if you were to develop them.

On these scales:

$$
\begin{aligned}
& 0=\text { would indicate that the problem is not serious at all } \\
& 1=\text { not serious enough to be worrying } \\
& 2=\text { moderately serious } \\
& 3=\text { very serious } \\
& 4=\text { extremely serious }
\end{aligned}
$$

If you are unable to rate the seriousness of a problem because you are not sure what the problem is, please tick the box on the right-hand side.

Not serious
at all

1. High blood pressure

2. Stomach ulcer

3. Blindness

4. Ear infection

5. Kidney disease

6. Aching legs

7. Leukemia (cancer of the blood)

8. Gum disease

9. Bronchitis

10. Deafness

(complẹte loss of hearing)

11. Numbness in the feet

12. Heart disease

13. Asthma

14. Failing eyesight

15. Loss of hearing (partly deaf)

16. Gangrene

17. Your diabetes now

18. Your diabetes in 10 years
Extremely

Serious
Not sure what the problem is 


\section{Diabetes Health Beliefs}

(Vulnerability)

In this section we are asking you to make two ratings for each of the problems listed.

First: $\quad$ Consider an average person with your kind of diabetes who is

- your age

- your sex

- follows the same kind of treatment as yourself

- has average control over her or his diabetes and indicate how likely you feel it is that this person will develop the following problems.

Second: Indicate how likely you feel it is that you will develop the following problems.

On these scales: $0=$ would indicate that you feel the development of the problem is very unlikely

$1=$ quite unlikely

$2=$ neither likely nor unlikely

3 = quite likely

$4=$ very likely

If you already have or think you may have any of these problems, please tick the box on the right-hand side.

1. High blood pressure

$\begin{array}{llr}\text { Very } & \text { Very } & \text { I already have } \\ \text { unlikely } & \text { likely } & \text { this problem }\end{array}$

Average person with

$\begin{array}{lllll}0 & 1 & 2 & 3 & 4\end{array}$

your kind of diabetes

Yourself

0

2. Stomach ulcer

Average person with your kind of diabetes

$\begin{array}{lllll}0 & 1 & 2 & 3 & 4\end{array}$

Yourself

0

1

2

34

\section{Blindness}

$\begin{array}{llllll}\begin{array}{l}\text { Average person with } \\ \text { your kind of diabetes }\end{array} & 0 & 1 & 2 & 3 & 4 \\ \text { Yourself } & 0 & 1 & 2 & 3 & 4\end{array}$

4. Ear infection

$\begin{array}{llllll}\begin{array}{l}\text { Average person with } \\ \text { your kind of diabetes }\end{array} & 0 & 1 & 2 & 3 & 4 \\ \begin{array}{l}\text { Yourself } \\ \text { yisease }\end{array} & 0 & 1 & 2 & 3 & 4 \\ \begin{array}{l}\text { Average person with } \\ \text { your kind of diabetes }\end{array} & 0 & 1 & 2 & 3 & 4 \\ \text { Yourself } & 0 & 1 & 2 & 3 & 4\end{array}$




$\begin{array}{llr}\begin{array}{l}\text { Very } \\ \text { unlikely }\end{array} & \begin{array}{l}\text { Very } \\ \text { likely }\end{array} & \begin{array}{r}\text { I already have } \\ \text { this problem }\end{array}\end{array}$

6. Aching legs

$\begin{array}{llllll}\begin{array}{l}\text { Average person with } \\ \text { your kind of diabetes }\end{array} & 0 & 1 & 2 & 3 & 4 \\ \text { Yourself } & 0 & 1 & 2 & 3 & 4\end{array}$

7. Leukemia

(cancer of the blood)

$\begin{array}{lllllll}\begin{array}{l}\text { Average person with } \\ \text { your kind of diabetes }\end{array} & 0 & 1 & 2 & 3 & 4\end{array}$

$\begin{array}{llllll}\text { Yourself } & 0 & 1 & 2 & 3 & 4\end{array}$

8. Gum disease

$\begin{array}{lllllll}\begin{array}{l}\text { Average person with } \\ \text { your kind of diabetes }\end{array} & 0 & 1 & 2 & 3 & 4\end{array}$

$\begin{array}{lllllll}\text { Yourself } & 0 & 1 & 2 & 3 & 4\end{array}$

9. Bronchitis

$\begin{array}{llllll}\begin{array}{l}\text { Average person with } \\ \text { your kind of diabetes }\end{array} & 0 & 1 & 2 & 3 & 4 \\ \text { Yourself } & 0 & 1 & 2 & 3 & 4\end{array}$

10. Deafness

(complete loss of hearing)

Average person with your kind of diabetes

Yourself

$\begin{array}{lllll}0 & 1 & 2 & 3 & 4\end{array}$

11. Numbness in the feet

Average person with your kind of diabetes

Yourself

$\begin{array}{lllll}0 & 1 & 2 & 3 & 4\end{array}$

12. Heart disease

Average person with your kind of diabetes

Yourself

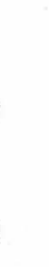

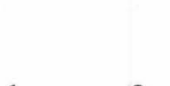




$\begin{array}{ccc}\text { Very } & \text { Very } & \text { I already have } \\ \text { unlikely } & \text { likely } & \text { this problem }\end{array}$

14. Failing eyesight

$\begin{array}{llllll}\begin{array}{l}\text { Average person with } \\ \text { your kind of diabetes }\end{array} & 0 & 1 & 2 & 3 & 4 \\ \text { Yourself } & 0 & 1 & 2 & 3 & 4\end{array}$

15. Loss of hearing (partly deaf)

$\begin{array}{llllll}\begin{array}{l}\text { Average person with } \\ \text { your kind of diabetes }\end{array} & 0 & 1 & 2 & 3 & 4 \\ \text { Yourself } & 0 & 1 & 2 & 3 & 4\end{array}$

16. Gangrene

$\begin{array}{llllll}\begin{array}{l}\text { Average person with } \\ \text { your kind of diabetes }\end{array} & 0 & 1 & 2 & 3 & 4 \\ \text { Yourself } & 0 & 1 & 2 & 3 & 4\end{array}$

17. Complications arising from diabetes

$\begin{array}{llllll}\begin{array}{l}\text { Average person with } \\ \text { your kind of diabetes }\end{array} & 0 & 1 & 2 & 3 & 4 \\ \text { Yourself } & 0 & 1 & 2 & 3 & 4\end{array}$




\section{OPINIONS ABOUT EXERCISING}

(Outcome Expectancy)

Please indicate how much you agree or disagree with each of the following statements. Rate your agreement by choosing any number between 0 and 100 using the scale below as a guide.

**Please note that the terms "exercise" and "exercising" can also be defined as "physical activity or being physically active."

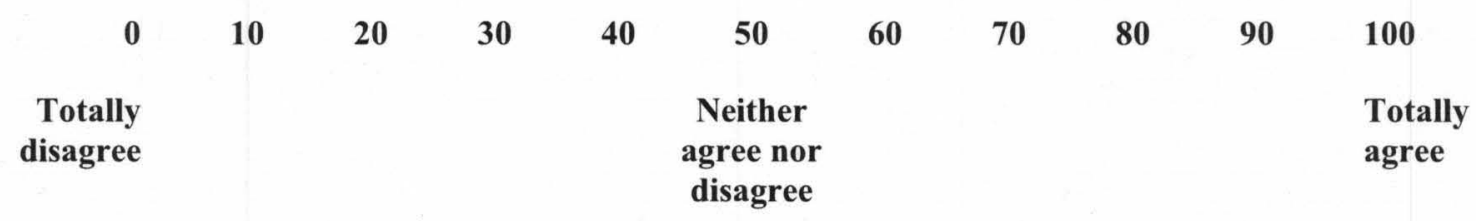

Agreement Rating

1. Exercising will disrupt my daily schedule.

2. Exercising regularly will make me feel healthier.

3. Exercising regularly will reduce my chances of developing chronic health problems.

4. I will get sore if I exercise. 


\section{CONFIDENCE IN EXERCISE}

(Task Self-Efficacy)

Please answer the following question by circling your confidence for each frequency (i.e., number of times) asked below.

**Please note that the terms "exercise" and "exercising" can also be defined as "physical activity or being physically active."

How confident are you that you can exercise for $\mathbf{3 0}$ accumulated minutes at a moderate, but comfortable, intensity...

A) 2 times per week?

$\begin{array}{lccccccccc}1 \quad 2 & 3 & 4 & 5 & 6 & 7 & 8 & 9 & 10 \\ \begin{array}{l}\text { Not at all } \\ \text { Confident }\end{array} & & & \text { Confident } & & & & \begin{array}{c}\text { Very } \\ \text { Confident }\end{array} \\ & & & & & & & & \end{array}$

B) 3 times per week?

$\begin{array}{lccccccccc}1 \quad 2 & 3 & 4 & 5 & 6 & 7 & 8 & 9 & 10 \\ \begin{array}{l}\text { Not at all } \\ \text { Confident }\end{array} & & & \begin{array}{c}\text { Confident } \\ \text { Very } \\ \text { Confident }\end{array}\end{array}$

C) 4 times per week?

$\begin{array}{lcllccllll}1 & 2 & 3 & 4 & 5 & 6 & 7 & 8 & 9 & 10 \\ \begin{array}{l}\text { Not at all } \\ \text { Confident }\end{array} & & & \begin{array}{c}\text { Confident } \\ \text { Cery } \\ \text { Confident }\end{array}\end{array}$

D) 5 times per week?

\begin{tabular}{|c|cccccccc}
$1 \quad 2$ & 3 & 4 & 5 & 6 & 7 & 8 & 9 & 10 \\
$\begin{array}{l}\text { Not at all } \\
\text { Confident }\end{array}$ & & & Confident & & & & $\begin{array}{c}\text { Very } \\
\text { Confident }\end{array}$ \\
\end{tabular}




\section{EXERCISE INTENTION}

(Behavioral Intention)

Please answer the following question regarding your intention to be physically active (or exercising) by circling the number that best describes your answer.

**Please note that the terms "exercise" and "exercising" can also be defined as "physical activity or being physically active."

1. My goal during the next 3 weeks is to exercise for 30 accumulated minutes at a moderate intensity

$\begin{array}{llccll}1 & 2 & 3 & 4 & 5 & 6\end{array}$

2. I intend to exercise for 30 accumulated minutes at a moderate intensity, 3 to 5 times per week, over the next 3 weeks.

$\begin{array}{lccccc}1 & 3 & 4 & 5 & 6 & 7 \\ \text { STRONGLY } & & & & & \text { STRONGLY } \\ \text { DISAGREE } & & & & & \text { AGREE }\end{array}$




\section{CONFIDENCE IN OVERCOMING EXERCISE BARRIERS}

(Barrier Self-efficacy)

The items below reflect common reasons preventing people from participating in physical activity. Using the scale below, please indicate (circle) how confident you are that you could be physically active in the event that any of the following circumstances were to occur. For example, if you have complete confidence that you can continue to exercise (be physically active), even if you are bored by the activity, you would circle $100 \%$. However, if you are absolutely sure that you could not exercise if you failed to make or continue to make progress you would circle $0 \%$ (No confidence at all).

**Please note that the terms "exercise" and "exercising" can also be defined as "physical activity or being physically active."

\section{I believe that I can exercise 3 times per week if:}

1) The weather is very bad (hot, humid, rainy, snow, cold).

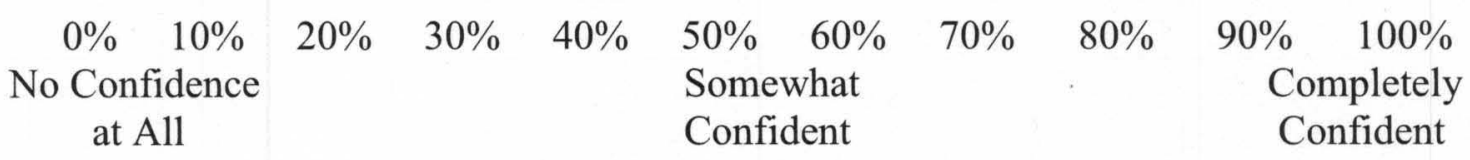

2) I was bored by the (exercise) program or activity.

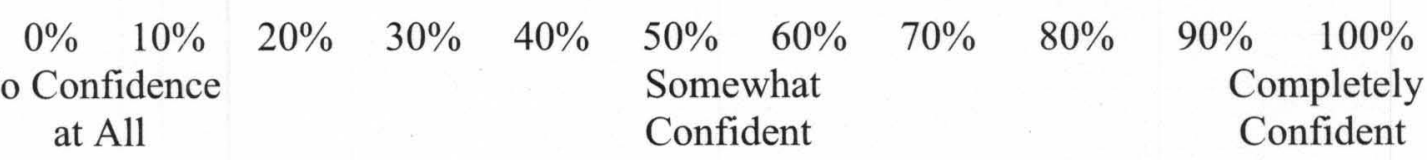

3) I was on vacation.

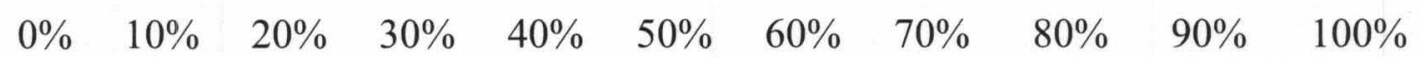

No Confidence Somewhat Completely

at All Confident Confident

4) I felt pain or discomfort when exercising.
$0 \% \quad 10 \%$
Confidence
$40 \%$
$50 \% \quad 60 \%$
$70 \%$
$80 \%$
Somewhat
Confident
$90 \% \quad 100 \%$
at All
Completely
Confident

5) I had to exercise alone.

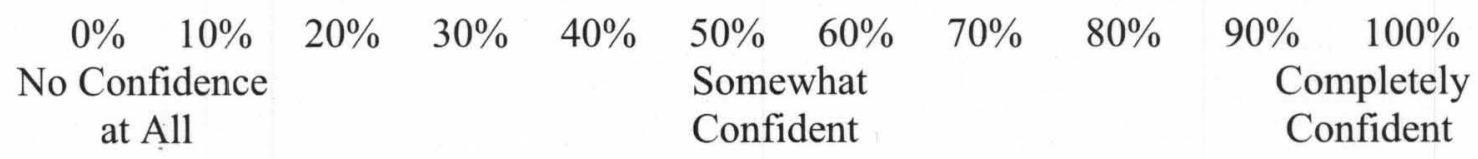


6) Exercise was not enjoyable or fun.

$\begin{array}{lllllllllll}0 \% & 10 \% & 20 \% & 30 \% & 40 \% & 50 \% & 60 \% & 70 \% & 80 \% & 90 \% & 100 \%\end{array}$

No Confidence

at All

Somewhat

Completely

Confident

Confident

7) It became difficult to get to the exercise location (i.e., where you exercise).

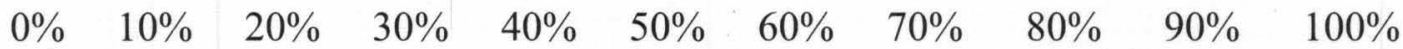

No Confidence $\quad$ Somewhat Completely

at All Confident Confident

8) I didn't like the particular activity program that I was involved in.

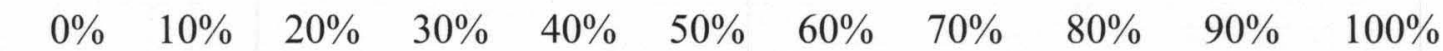

No Confidence Somewhat Completely

at All Confident Confident

9) My work schedule conflicted with my exercise session.

$\begin{array}{lllllllllll}0 \% & 10 \% & 20 \% & 30 \% & 40 \% & 50 \% & 60 \% & 70 \% & 80 \% & 90 \% & 100 \%\end{array}$

No Confidence Somewhat Completely
at All
Confident
Confident

10) I felt self-conscious about my appearance when I exercised.

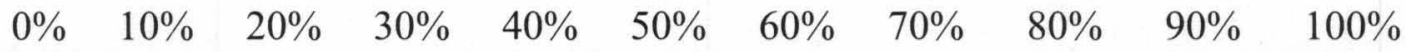

No Confidence $\quad$ Somewhat Completely
at All Confident Confident

11) The instructor (of an exercise program) did not offer me any encouragement.

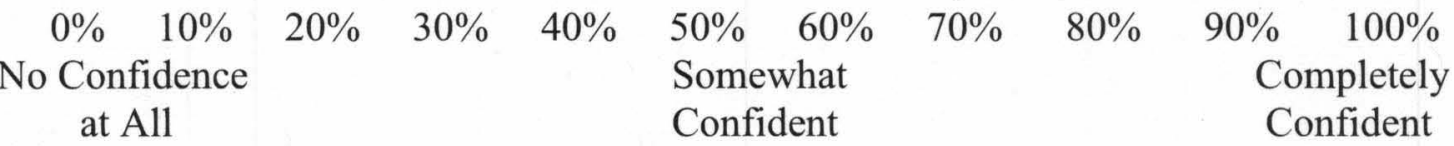

12) I was under personal stress of some kind.

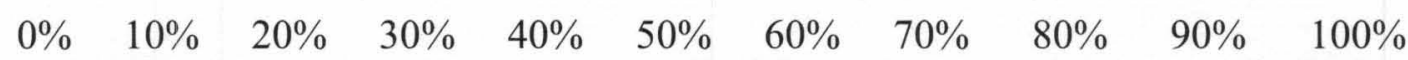

No Confidence Somewhat Completely

at All Confident Confident




\section{EXERCISE PLANNING}

(Action Planning)

Please read the statement below for each item (A through D) and answer each by circling how true or untrue this statement applied to you.

I have made a detailed plan regarding...

A) when to do my physical activity

$\begin{array}{cccc}1 & 2 & 3 & 4 \\ \begin{array}{c}\text { Not at all } \\ \text { True }\end{array} & & & \text { Exactly } \\ & & \text { True }\end{array}$

B) where to do my physical activity

$\begin{array}{cccc}1 & 2 & 3 & 4 \\ \begin{array}{c}\text { Not at all } \\ \text { True }\end{array} & & & \text { Exactly } \\ \text { True }\end{array}$

C) how to do my physical activity

(for example: walking, jogging, bicycling, swimming, other, etc.)

$\begin{array}{cccc}1 & 2 & 3 & 4 \\ \begin{array}{c}\text { Not at all } \\ \text { True }\end{array} & & & \begin{array}{c}\text { Exactly } \\ \text { True }\end{array}\end{array}$

D) how often to do my physical activity

$\begin{array}{cccc}1 & 2 & 3 & 4 \\ \begin{array}{c}\text { Not at all } \\ \text { True }\end{array} & & & \text { Exactly } \\ \text { True }\end{array}$




\section{PHYSICAL ACTIVITY QUESTIONNAIRE \\ (International Physical Activity Questionnaire)}

We are interested in finding out about the kinds of physical activities that people do as part of their everyday lives. The questions will ask you about the time you spent being physically active in the last 7 days. Please answer each question even if you do not consider yourself to be an active person. Please think about the activities you do at work, as part of your house and yard work, to get from place to place, and in your spare time for recreation, exercise or sport.

Think about all the vigorous and moderate activities that you did in the last 7 davs. Vigorous physical activities refer to activities that take hard physical effort and make you breathe much harder than normal. Moderate activities refer to activities that take moderate physical effort and make you breathe somewhat harder than normal.

\section{PART 1: JOB-RELATED PHYSICAL ACTIVITY}

The first section is about your work. This includes paid jobs, farming, volunteer work, course work, and any other unpaid work that you did outside your home. Do not include unpaid work you might do around your home, like housework, yard work, general maintenance, and caring for your family. These are asked in Part 3.

1. Do you currently have a job or do any unpaid work outside your home?

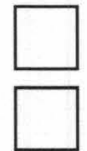

Yes

No

Skip to PART 2: TRANSPORTATION

The next questions are about all the physical activity you did in the last $\mathbf{7}$ days as part of your paid or unpaid work. This does not include traveling to and from work.

2. During the last $\mathbf{7}$ days, on how many days did you do vigorous physical activities like heavy lifting, digging, heavy construction, or climbing up stairs as part of your work? Think about only those physical activities that you did for at least 10 minutes at a time.

\section{days per week}

No vigorous job-related physical activity

Skip to question 4

3. How much time did you usually spend on one of those days doing vigorous physical activities as part of your work?

hours per day

minutes per day 
4. Again, think about only those physical activities that you did for at least 10 minutes at a time. During the last $\mathbf{7}$ days, on how many days did you do moderate physical activities like carrying light loads as part of your work? Please do not include walking.

\section{days per week}

No moderate job-related physical activity

5. How much time did you usually spend on one of those days doing moderate physical activities as part of your work?

\section{hours per day \\ minutes per day}

6. During the last 7 days, on how many days did you walk for at least 10 minutes at a time as part of your work? Please do not count any walking you did to travel to or from work.

\section{days per week}

No job-related walking

7. How much time did you usually spend on one of those days walking as part of your work?

hours per day

minutes per day

\section{PART 2: TRANSPORTATION PHYSICAL ACTIVITY}

These questions are about how you traveled from place to place, including to places like work, stores, movies, and so on.

8. During the last $\mathbf{7}$ days, on how many days did you travel in a motor vehicle like a train, bus, car, or tram?

\section{days per week}

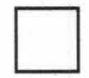

No traveling in a motor vehicle

Skip to question 10

9. How much time did you usually spend on one of those days traveling in a train, bus, car, tram, or other kind of motor vehicle?

hours per day

minutes per day 
Now think only about the bicycling and walking you might have done to travel to and from work, to do errands, or to go from place to place.

10. During the last 7 days, on how many days did you bicycle for at least 10 minutes at a time to go from place to place?

days per week

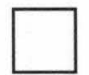

No bicycling from place to place

Skip to question 12

11. How much time did you usually spend on one of those days to bicycle from place to place?

hours per day
$-\quad$ minutes per day

12. During the last $\mathbf{7}$ days, on how many days did you walk for at least 10 minutes at a time to go from place to place?

days per week

No walking from place to place

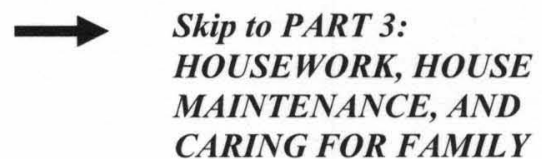

13. How much time did you usually spend on one of those days walking from place to place?

hours per day

minutes per day

\section{PART 3: HOUSEWORK, HOUSE MAINTENANCE, AND CARING FOR FAMILY}

This section is about some of the physical activities you might have done in the last $\mathbf{7}$ days in and around your home, like housework, gardening, yard work, general maintenance work, and caring for your family.

14. Think about only those physical activities that you did for at least 10 minutes at a time. During the last 7 days, on how many days did you do vigorous physical activities like heavy lifting, chopping wood, shoveling snow, or digging in the garden or yard?

\section{days per week}

No vigorous activity in garden or yard

Skip to question 16

15. How much time did you usually spend on one of those days doing vigorous physical activities in the garden or yard?

hours per day

minutes per day 
16. Again, think about only those physical activities that you did for at least 10 minutes at a time. During the last 7 days, on how many days did you do moderate activities like carrying light loads, sweeping, washing windows, and raking in the garden or yard?

\section{days per week}

No moderate activity in garden or yard

Skip to question 18

17. How much time did you usually spend on one of those days doing moderate physical activities in the garden or yard?

hours per day

minutes per day

18. Once again, think about only those physical activities that you did for at least 10 minutes at a time. During the last 7 days, on how many days did you do moderate activities like carrying light loads, washing windows, scrubbing floors and sweeping inside your home?

\section{days per week}

No moderate activity inside home

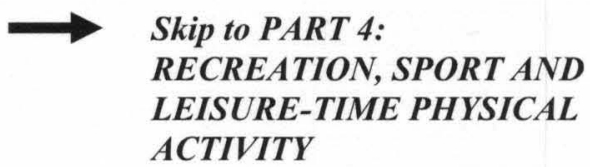

19. How much time did you usually spend on one of those days doing moderate physical activities inside your home?
hours per day
minutes per day

PART 4: RECREATION, SPORT, AND LEISURE-TIME PHYSICAL ACTIVITY

This section is about all the physical activities that you did in the last $\mathbf{7}$ days solely for recreation, sport, exercise or leisure. Please do not include any activities you have already mentioned.

20. Not counting any walking you have already mentioned, during the last $\mathbf{7}$ days, on how many days did you walk for at least 10 minutes at a time in your leisure time?

\section{days per week}

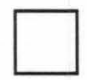

No walking in leisure time

Skip to question 22

21. How much time did you usually spend on one of those days walking in your leisure time?

hours per day

minutes per day 
22. Think about only those physical activities that you did for at least 10 minutes at a time. During the last 7 days, on how many days did you do vigorous physical activities like aerobics, running, fast bicycling, or fast swimming in your leisure time?

\section{days per week}

No vigorous activity in leisure time

Skip to question 24

23. How much time did you usually spend on one of those days doing vigorous physical activities in your leisure time?

\section{hours per day}

minutes per day

24. Again, think about only those physical activities that you did for at least 10 minutes at a time. During the last 7 days, on how many days did you do moderate physical activities like bicycling at a regular pace, swimming at a regular pace, and doubles tennis in your leisure time?

\section{days per week}

No moderate activity in leisure time

Skip to PART 5: TIME SPENT SITTING

25. How much time did you usually spend on one of those days doing moderate physical activities in your leisure time?

hours per day

minutes per day

\section{PART 5: TIME SPENT SITTING}

The last questions are about the time you spend sitting while at work, at home, while doing course work and during leisure time. This may include time spent sitting at a desk, visiting friends, reading or sitting or lying down to watch television. Do not include any time spent sitting in a motor vehicle that you have already told me about.

26. During the last $\mathbf{7}$ days, how much time did you usually spend sitting on a weekday?

hours per day

minutes per day

27. During the last $\mathbf{7}$ days, how much time did you usually spend sitting on a weekend day?

hours per day

minutes per day 


\section{PHYSICAL ACTIVITY QUESTIONNAIRE (Interviewer Administered IPAQ Telephone Long Form)}

READ: I am going to ask you about the time you spent being physically active in the last 7 days. Please answer each question even if you do not consider yourself to be an active person. Think about the activities you do at work, as part of your house and yard work, to get from place to place, and in your spare time for recreation, exercise or sport.

PART 1: JOB-RELATED PHYSICAL ACTIVITY

READ: The first questions are about your work. This includes paid jobs, farming, volunteer work, course work and any other unpaid work that you did outside your home. Do not include unpaid work you might do around your home, like housework, yard work, general maintenance, and caring for your family. I will ask you about these later.

1. Do you currently have a job or do any unpaid work outside your home?

Yes

$\begin{array}{ll}\text { N. } & \text { No [Skip to PART 2] } \\ \text { 9. } & \text { Don't Know/Not Sure [Skip to PART 2] } \\ & \text { Refused [Skip to PART 2] }\end{array}$

[Interviewer clarification: This also includes credit and non-credit classes or course work. It also includes volunteer work and time spent looking for work. It does not includes unpaid house or yard work, nor caring for dependents, this will be asked in a later section.]

READ: The following questions are about all the physical activity you did as part of your paid or unpaid work. This does not include traveling to and from work.

READ: First, think about all the vigorous activities which take hard physical effort that you did as part of your work. Vigorous activities make you breathe much harder than normal. These may include things like heavy lifting, digging, heavy construction work, or climbing up stairs. Think about only those vigorous physical activities that you did for at least 10 minutes at a time.

2. During the last 7 days, on how many days did you do vigorous physical activities as part of your work?

\begin{tabular}{ll} 
& Days per week [If respondent answers 0, skip to Question 4] \\
\hline 8. & Don't Know/Not Sure [Skip to Question 4] \\
9. & Refused [Skip to Question 4]
\end{tabular}

[Interviewer clarification: Think about only those physical activities that you did for at least 10 minutes at a time.]

[Interviewer clarification: Work includes paid and unpaid work as well as course work. Include all jobs and volunteer work.] 
3. How much time did you usually spend on one of those days doing vigorous physical activities as part of your work?

\begin{tabular}{|c|c|}
\hline - & $\begin{array}{l}\text { Hours per day } \\
\text { Minutes per day }\end{array}$ \\
\hline & Don't Know/Not Sure \\
\hline & Refused \\
\hline
\end{tabular}

[Interviewer clarification: Think about only those physical activities you did for at least $\mathbf{1 0}$ minutes at a time.]

[Interviewer probe: An average time per day is being sought. If the respondent can't answer because the pattern of time spent varies widely from day to day, or includes time spent doing a variety of paid and unpaid work, ask: "What is the total amount of time you spent over the last 7 days doing vigorous physical activities as part of your work?"

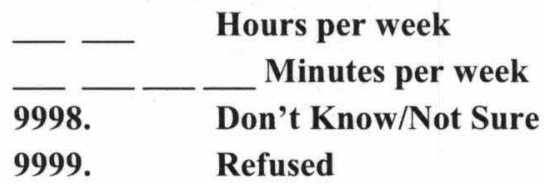

READ: Now think about activities which take moderate physical effort that you did as part of you work. Moderate physical activities make you breathe somewhat harder than normal and may include activities like carrying light loads. Do not include walking. Again, think about only those moderate physical activities that you did for at least 10 minutes at a time.

4. During the last 7 days, on how many days did you do moderate physical activities as part of your work?
8. Don't Know/Not Sure [Skip to Question 6]
9. $\quad$ Refused [Skip to Question 6]

[Interviewer clarification: Think about only those physical activities that you did for at least 10 minutes at a time.]

[Interviewer clarification: Work includes paid and unpaid work as well as course work. Include all jobs.]

5. How much time did you usually spend on one of those days doing moderate physical activities as part of your work?

$\begin{array}{ll}-- & \begin{array}{l}\text { Hours per day } \\ \text { Minutes per day }\end{array} \\ 998 . & \begin{array}{l}\text { Don't Know/Not Sure } \\ \text { Refused }\end{array} \\ 999 . & \quad \text { Refused }\end{array}$

[Interviewer clarification: Think about only those physical activities you did for at least $\mathbf{1 0}$ minutes at a time.] 
[Interviewer probe: An average time per day is being sought. If the respondent can't answer because the pattern of time spent varies widely from day to day, or includes time spent doing a variety of paid and unpaid work, ask: "What is the total amount of time you spent over the last 7 days doing moderate physical activities as part of your work?"

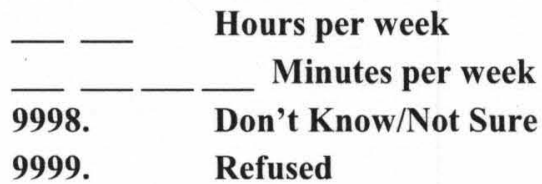

READ: Now think about the time you spend walking for at least 10 minutes at a time as part of your work. Please do not count any walking you did to travel to or from work.

6. During the last 7 days, on how many days did you walk as part of your work?

Days per week [If respondent answers 0, skip to PART 2]

8. Don't Know/Not Sure [Skip to PART 2]

9. Refused [Skip to PART 2]

[Interviewer clarification: Think about only the walking that you did for at least 10 minutes at a time.]

[Interviewer clarification: Include all jobs.]

7. How much time did you usually spend on one of those days walking as part of your work?

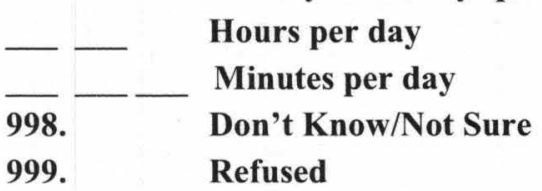

[Interviewer clarification: Think about only the walking you did for at least 10 minutes at a time.]

[Interviewer probe: An average time per day is being sought. If the respondent can't answer because the pattern of time spent varies widely from day to day, or includes time spent doing a variety of paid and unpaid work, ask: "What is the total amount of time you spent walking over the last 7 days as part of your work?"

$\begin{array}{ll}-- & \begin{array}{l}\text { Hours per week } \\ \text { M998. }\end{array}-\begin{array}{l}\text { Minutes per week } \\ \text { Don't Know/Not Sure } \\ \text { Refused }\end{array} \\ 9999 . & \quad \text { Ro }\end{array}$


READ: Now, think about how you traveled from place to place, including to places like work, stores, movies and so on.

8. During the last 7 days, on how many days did you travel in a motor vehicle like a train, bus, car or tram?

\begin{tabular}{ll} 
& Days per week [If respondent answer 0, skip to Question 10] \\
\hline 8. & Don't Know/Not Sure [Skip to Question 10] \\
9. & Refused [Skip to Question 10]
\end{tabular}

9. How much time did you usually spend on one of those days traveling in a car, bus, train or other kind of motor vehicle?

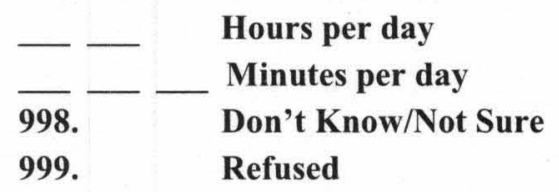

[Interviewer probe: An average time per day is being sought. If the respondent can't answer because the pattern of time spent varies widely from day to day, ask: "What is the total amount of time you spent over the last 7 days traveling in a motor vehicle?"

$$
\begin{array}{ll}
-- & \begin{array}{l}
\text { Hours per week } \\
\text { Minutes per week }
\end{array} \\
9998 . & \begin{array}{l}
\text { Don't Know/Not Sure } \\
\text { Refused }
\end{array} \\
9999 . & \quad
\end{array}
$$

READ: Now think only about the bicycling you did to travel to and from work, to do errands, or to go from place to place. Only include bicycling that you did for at least 10 minutes at a time.

10. During the last 7 days, on how many days did you bicycle to go from place to place?
8.
Days per week [If respondent answers 0, skip to Question 12]
8. Don't Know/Not Sure [Skip to Question 12]
9. $\quad$ Refused [Skip to Question 12]

[Interviewer clarification: Think only about the bicycling that you did for at least 10 minutes at a time.]

11. How much time did you usually spend on one of those days to bicycle from place to place?

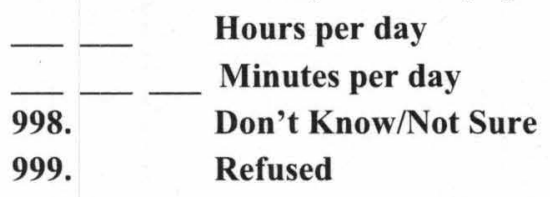

[Interviewer clarification: Think about only the bicycling that you did for at least 10 minutes at a time.] 
[Interviewer probe: An average time per day is being sought. If the respondent can't answer because the pattern of time spent varies widely from day to day, ask: "What is the total amount of time you spent bicycling over the last 7 days to travel from place to place?"

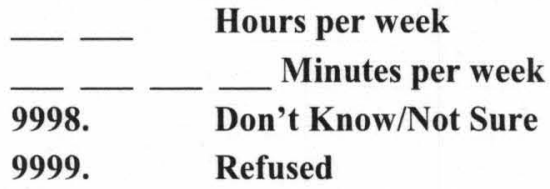

READ: Now think only about the walking you did to travel to and from work, to do errands or to go from place to place. Only include walking that you did for at least 10 minutes at a time.

12. During the last 7 days, on how many days did you walk to go from place to place?
Days per week [If respondent answers 0, skip to PART 3]
8. Don't Know/Not Sure [Skip to PART 3]
9. Refused [Skip to PART 3]

[Interviewer clarification: Think only about the walking that you did for at least $\mathbf{1 0}$ minutes at a time.]

13. How much time did you usually spend on one of those days walking from place to place?

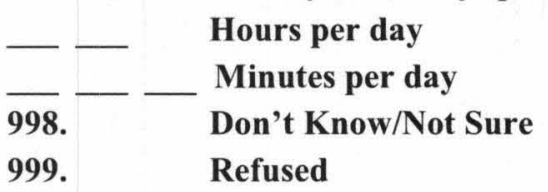

[Interviewer clarification: Think about only the walking that you did for at least $\mathbf{1 0}$ minutes at a time.]

[Interviewer probe: An average time per day is being sought. If the respondent can't answer because the pattern of time spent varies widely from day to day, ask: "What is the total amount of time you spent over the last 7 days walking from place to place?"

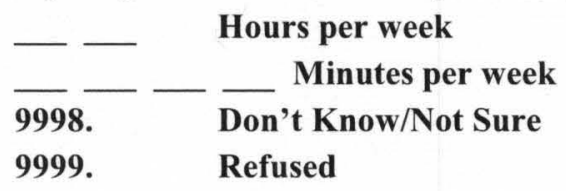

PART 3: HOUSEWORK, HOUSE MAINTENANCE AND CARING FOR FAMILY

READ: Now think about the physical activities you have done in the last 7 days in and around your home, like housework, gardening, yard work, general maintenance work, and caring for your family.

READ: First think about vigorous activities which take hard physical effort that you did in the garden or yard. Vigorous activities make you breathe much harder than normal and may include heavy lifting, chopping wood, shoveling snow, or digging. Again, think about only those vigorous physical activities that you did for at least 10 minutes at a time. 
14. During the last 7 days, on how many days did you do vigorous physical activities in the garden or yard?

$\begin{array}{ll} & \text { Days per week [If respondent answers 0, skip to Question 16] } \\ \text { 8. } & \text { Don't Know/Not Sure [Skip to Question 16] } \\ 9 . & \text { Refused [Skip to Question 16] }\end{array}$

[Interviewer clarification: Think about only those physical activities that you did for at least 10 minutes at a time.]

15. How much time did you usually spend on one of those days doing vigorous physical activities in the garden or yard?

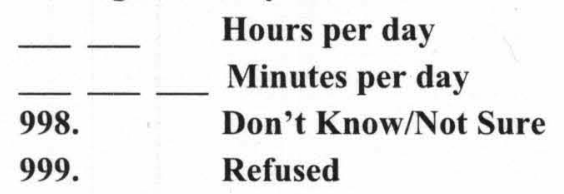

[Interviewer clarification: Think about only those physical activities that you did for at least 10 minutes at a time.]

[Interviewer probe: An average time per day is being sought. If the respondent can't answer because the pattern of time spent varies widely from day to day, ask: "What is the total amount of time you spent over the last 7 days doing vigorous physical activities in the garden or yard?"

\begin{tabular}{|c|c|}
\hline- & $\begin{array}{l}\text { Hours per week } \\
\text { Minutes per week }\end{array}$ \\
\hline 98. & $\begin{array}{l}\text { Don't Know/Not Sure } \\
\text { Refused }\end{array}$ \\
\hline
\end{tabular}

READ: Now think about activities which take moderate physical effort that you did in the garden or yard. Moderate physical activities make you breathe somewhat harder than normal and may include carrying light loads, sweeping, washing windows, and raking. Again, include only those moderate physical activities that you did for at least 10 minutes at a time.

16. During the last 7 days, on how many days did you do moderate activities in the garden or yard?

$\begin{array}{ll} & \text { Days per week [If respondent answers 0, skip to Question 18] } \\ \text { 8. } & \text { Don't Know/Not Sure [Skip to Question 18] } \\ \text { 9. } & \text { Refused [Skip to Question 18] }\end{array}$

[Interviewer clarification: Think about only those physical activities that you did for at least 10 minutes at a time.]

17. How much time did you usually spend on one of those days doing moderate physical activities in the garden or yard?

$\begin{array}{ll}-- & \begin{array}{l}\text { Hours per day } \\ \text { Minutes per day }\end{array} \\ 998 . & \begin{array}{l}\text { Don't Know/Not Sure } \\ \text { Refused }\end{array} \\ 999 . & \end{array}$

[Interviewer clarification: Think about only those physical activities that you did for at least 10 minutes at a time.] 
[Interviewer probe: An average time per day is being sought. If the respondent can't answer because the pattern of time spent varies widely from day to day, ask: "What is the total amount of time you spent over the last 7 days doing moderate physical activities in the garden or yard?"

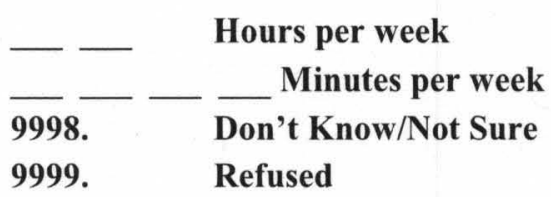

READ: Now think about activities which take at least moderate physical effort that you did inside your home. Examples include carrying light loads, washing windows, scrubbing floors, and sweeping. Include only those moderate physical activities that you did for at least 10 minutes at a time.

[Interviewer clarification: Moderate activities make you breathe somewhat harder than normal.]

18. During the last 7 days, on how many days did you do moderate activities inside your home?

\begin{tabular}{ll} 
& Days per week [If respondent answers 0, \\
\hline 8. & Don't Know/Not Sure [Skip to PART 4] \\
9. & Refused [Skip to PART 4]
\end{tabular}

[Interviewer clarification: Think about only those physical activities that you did for at least 10 minutes at a time.]

[Interviewer clarification: During the last 7 days, on how many days did you do activities that take at least moderate effort inside your home?]

19. How much time did you usually spend on one of those days doing moderate physical activities inside your home?

$\begin{array}{ll}- & -\quad \begin{array}{l}\text { Hours per day } \\ \text { Minutes per day } \\ \text { 998. }\end{array} \quad \begin{array}{l}\text { Don't Know/Not Sure } \\ \text { Refused }\end{array} \\ 999 . & \text { a }\end{array}$

[Interviewer clarification: Think about only those physical activities that you did for at least 10 minutes at a time.]

[Interviewer probe: An average time per day is being sought. If the respondent can't answer because the pattern of time spent varies widely from day to day, ask: "What is the total amount of time you spent over the last 7 days doing moderate physical activities inside your home?"

\begin{tabular}{|c|c|}
\hline$\longrightarrow$ & $\begin{array}{l}\text { Hours per week } \\
\quad \text { Minutes per week }\end{array}$ \\
\hline & Don't Know/Not Sure \\
\hline
\end{tabular}


READ: Now, think about all the physical activities that you did in the last 7 days solely for recreation, sport, exercise or leisure. Please do not include any activities you have already mentioned.

20. Not counting any walking you have already mentioned, during the last 7 days, on how many days did you walk for at least $\mathbf{1 0}$ minutes at a time in your leisure time?
Days per week [If respondent answers 0, skip to Question 22]
$8 . \quad$ Don't Know/Not Sure [Skip to Question 22]
9. $\quad$ Refused [Skip to Question 22]

[Interviewer clarification: Think about only the walking that you did for at least $\mathbf{1 0}$ minutes at a time.]

21. How much time did you usually spend on one of those days walking in your leisure time?

$\begin{array}{ll}-- & \begin{array}{l}\text { Hours per day } \\ \text { Minutes per day }\end{array} \\ \mathbf{9 9 8} . & \begin{array}{l}\text { Don't Know/Not Sure } \\ \text { Refused }\end{array} \\ 999 . & \quad\end{array}$

[Interviewer clarification: Think about only the walking that you did for at least 10 minutes at a time.]

[Interviewer probe: An average time per day is being sought. If the respondent can't answer because the pattern of time spent varies widely from day to day, ask: "What is the total amount of time you spent over the last 7 days walking in your leisure time?"

\begin{tabular}{|c|c|}
\hline & Hours per week \\
\hline & __ Minutes per week \\
\hline 9999 & Refused \\
\hline
\end{tabular}

READ: Now think about other physical activities you did in your leisure time for at least 10 minutes at a time.

READ: First, think about vigorous activities which take hard physical effort that you did in your leisure time. Examples include aerobics, running, fast bicycling, or fast swimming.

[Interviewer clarification: Vigorous activities make you breathe much harder than normal.]

22. During the last 7 days, on how many days did you do vigorous physical activities in your leisure time?

\begin{tabular}{ll} 
& Days per week [If respondent answers 0, skip to Question 24] \\
\hline 8. & Don't Know/Not Sure [Skip to Question 24] \\
$9 . \quad$ & Refused [Skip to Question 24]
\end{tabular}

[Interviewer clarification: Think about only those vigorous physical activities that you did for at least 10 minutes at a time.] 
23. How much time did you usually spend on one of those days doing vigorous physical activities in your leisure time?

\begin{tabular}{|c|c|}
\hline$\ldots$ & $\begin{array}{l}\text { Hours per day } \\
\text { Minutes per day }\end{array}$ \\
\hline & Don't Know/Not Su \\
\hline 99 & Refused \\
\hline
\end{tabular}

READ: Now think about other physical activities you did in your leisure time for at least 10 minutes at a time.

READ: First, think about moderate activities which take hard physical effort that you did in your leisure time. Examples include bicycling at a regular pace, swimming at a regular pace, and doubles tennis.

24. Again, think about only those physical activities that you did for at least 10 minutes at a time. During the last $\mathbf{7}$ days, on how many days did you do moderate physical activities like bicycling at a regular pace, swimming at a regular pace, and doubles tennis in your leisure time?

\section{Don't Know/Not Sure [Skip to Question 22] \\ 9. $\quad$ Refused [Skip to Question 22]}

25. How much time did you usually spend on one of those days doing moderate physical activities in your leisure time?

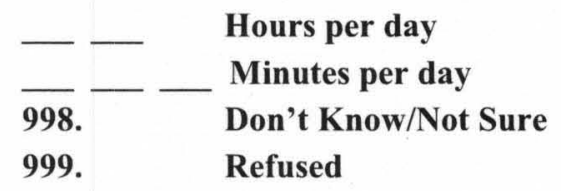

\section{PART 5: TIME SPENT SITTING}

The last questions are about the time you spend sitting while at work, at home, while doing course work and during leisure time. This may include time spent sitting at a desk, visiting friends, reading or sitting or lying down to watch television. Do not include any time spent sitting in a motor vehicle that you have already told me about.

26. During the last 7 days, how much time did you usually spend sitting on a weekday?

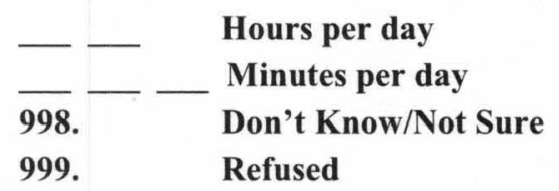

27. During the last 7 days, how much time did you usually spend sitting on a weekend day?

\begin{tabular}{|c|c|}
\hline - - & $\begin{array}{l}\text { Hours per day } \\
\text { Minutes per day }\end{array}$ \\
\hline & Don't Know/Not Sure \\
\hline 999. & Refused \\
\hline
\end{tabular}




\section{CURRICULUM VITAE}

\section{Jason Edward Bonner}

Contact Information

12302 Spring Meadow Drive

Chapel Hill, NC 27517

dr.jbsot@gmail.com

502-419-3759

\section{RESEARCH \& CLINICAL INTERESTS}

Broad research and clinical interests in Clinical Health Psychology including:

- Application of evidence-based practices \& theory driven interventions to lifestyle behavior change in chronic illness management, with specific interests in type 2 diabetes mellitus and chronic pain.

- Psychological adjustment to acute and chronic illness in medical settings, with specific emphasis in integrated primary care, home-based primary care, and medical specialty clinics.

- Adherence to medical treatment regimen, including measurement issues and enhancing patient-provider interactions to improve adherence.

- Mindfulness-based interventions for stress and chronic pain management.

\section{EDUCATION}

2010 (December)

2010

2005

2002
Ph.D., Clinical Psychology, University of Louisville

APA Accredited Clinical Psychology Internship Department of Veterans Affairs, Southern Arizona VAHCS, Tucson, Arizona

M.A., Psychology, University of Louisville

B.A., Psychology, University of Southern Maine 


\section{PROFESSIONAL AFFILIATIONS}

Society of Behavioral Medicine, 2003 to present

Association for Behavioral and Cognitive Therapies, 2003 to present

American Psychological Association, 2006 to present

American Psychological Association, Division 38: Health Psychology, 2006 to present

American Psychological Association of Graduate Students, 2006 to present

\section{CLINICAL EXPERIENCE}

2009-2010

Psychology Intern, Southern Arizona VA Healthcare System, Tucson, AZ. Expected Graduation: Aug. 17, 2010.

Rotations:

Chronic Pain Management Clinic: Responsible for assessment and treatment of psychological and behavioral health problems associated with chronic pain management. Responsibilities include initial evaluation for pain psychology treatments, evaluation for spinal cord stimulator implantation, individual \& group-based psychotherapy, participate and conduct weekly psychoeducation classes on chronic pain management topics, and develop and implement a mindfulness-based pain management group.

Supervisor: Andrew Jones, Ph.D.

Polytrauma Unit (VISN-18 Network Site): Responsible for conducting specialty psychological evaluation and management to veterans who experienced severe injuries to multiple organ systems, including Traumatic Brain Injury (TBI). Responsibilities include conducting comprehensive assessment, individual \& group psychotherapy, and assist in coordinating on-going case management. Supervisor: Anne Scott, Ph.D.

Blind Rehabilitation Service (Southwest Blind Rehabilitation Center-SWBRC): Provide psychological evaluation, individual, and group psychotherapy for blind and low-vision veterans and active-duty service members with emphasis on re-establishing functional independence and improved quality of life. Supervisor: Lisa Burgess, Ph.D.

Health Assessment/Psychological Evaluation: Provide psychological testing \& evaluation in-support of medical specialty and mental health clinic services. Assessments include bariatric \& organ transplantation, personality assessment, and cognitive screening. Test data is summarized and presented in an integrated assessment report. Supervisor: Julie Ewing, Ph.D. 
PTSD Inpatient \& Outpatient Rotation: Provide individual \& group treatments in outpatient mental health clinic. Additionally, participate in inpatient PTSD program: Evaluation \& Brief Treatment of PTSD Unit -EBTPU, delivering evidence-based individual \& group-based treatments to inpatient trauma survivors. Supervisors: Alan Baehr, Ph.D., David Beil-Adaskin, Ph.D., and Nadine Cole, Ph.D.

Long-term Outpatient Psychotherapy: Provide individual outpatient psychotherapy for veterans presenting with a variety of psychological and behavioral health difficulties. Specialty emphasis and training in Acceptance \& Commitment Therapy (ACT), Cognitive-Behavioral Therapy, Cognitive-Processing Therapy, and Behavioral interventions (e.g., exposure-based treatments). Additional duties include co-facilitating and leading clinic group therapy sessions as needed through the year (e.g., smoking cessation group, ACT group). Supervisors: Sabrina Hitt, Ph.D., Julie Ewing, Ph.D., and Anne Scott, Ph.D.

Supervision Rotation (Subset of Long-term Outpatient Psychotherapy Rotation): Provide weekly peer supervision to other psychology interns on long-term outpatient cases. Additional responsibilities: provide weekly supervision to undergraduate research assistants from the University of Arizona who are conducting federally-funded VA research on interactions of sleep, depression, and PTSD in veterans. Supervisors: Alan Baehr, Ph.D. and Patricia Haynes, Ph.D.

2006-2007

Graduate Student Therapist, Central State Hospital, Louisville, KY.

Provided individual and group therapy interventions and psychological assessment in an acute-care inpatient mental health setting. Specific responsibilities included suicide risk assessments and individual and group psychotherapy for mild to severe mentally ill patients. Supervisors: Jeff Hicks, Ph.D., Wayne J. Putnam, Psy.D.

Graduate Student Psychological Examiner, Private Practice of Dr. Steven Simon, Louisville, KY.

Conducted psychological and neuropsychological medical disability claims assessments referred by the Kentucky Department of Disability Determinations and Indiana Disability Determination Bureau. Supervisor: Steven Simon, Ph.D. 
Conducted in-home assessment and treatment for smoking cessation, sleep hygiene, medication and treatment adherence across a number of chronic illnesses, and address co-occurring psychological distress (i.e., depression, anxiety, etc.). Additional responsibilities included treatment review and planning at Interdisciplinary Team meetings. Supervisor: Barbara Stetson, Ph.D. Services Center, Department of Psychological and Brain Sciences, University of Louisville, Louisville, KY.

Provided psychological assessment and treatment using timelimited interpersonal psychotherapy with emphasis on the clienttherapist relationship and emotional re-learning. Supervisor: Stan Murrell, Ph.D.

2003-2005

Graduate Student Therapist, Ambulatory Internal Medicine Clinic, Department of Medicine, University of Louisville, Louisville, KY.

Provided individual therapy and psychological assessment in an integrated primary care setting serving a medically-underserved population. Specific responsibilities included screening patients for psychological and behavioral health difficulties including smoking cessation, sleep hygiene, weight management, medication and treatment adherence across a number of chronic illnesses, and cooccurring psychological distress (i.e., depression, anxiety). Supervisors: Abbie Beacham, Ph.D. and Amy Buckley, Ph.D.

\section{ADDITIONAL CLINICAL TRAINING}

Motivational Interviewing Training, Southern Arizona VA Healthcare System: $8+$ week seminar with ongoing case review and supervision in the clinical application of motivational interviewing (MI) in a variety of settings including behavioral medicine, substance abuse, and general mental illness.

Course Instructor: Sandra Gallagher, Ph.D.

2010 Prolonged Exposure Training, Southern Arizona VA Healthcare System: $8+$ week seminar with ongoing case review and supervision in the clinical application of prolonged exposure (PE) as a treatment for Post-Traumatic Stress Disorder.

Course Instructor: David Beil-Adaskin, Ph.D. 
Mindfulness and Psychotherapy online training program, National Institute for the Clinical Application of Behavioral Medicine (NICABM). Six-week online course in the history, theory, and clinical application of mindfulness-based interventions in psychotherapy. Course involved homestudy, online interactive training and conference call sessions led by course instructor/supervisor.

Course instructor: Ronald D. Siegel, Psy.D.

Four-day Mindfulness Retreat, University of Louisville.

Didactic and experiential training in mindfulness-based interventions including instruction in the history and fundamental principles of mindfulness-based psychotherapy, meditation, and yoga. Course instructor: Paul Salmon, Ph.D.

\section{PUBLICATIONS}

Rogers, W., Meyer, J. Rothschild, C., Bonner, J. Das, N., Richardson, K., Mokshagundam, S. P., Krishnasamy, S., Kong, M., Stetson, B. (2009). Social isolation is associated with geographic socioeconomic status and quality of life in at-risk, underserved adults with type 2 diabetes. Annals of Behavioral Medicine, 37 (Supplement), S199.

Richardson, K., Rogers, W., Bonner, J., Rothschild, C., Meyer, J., Das, N., Mokshagundam, S. P., Krishnasamy, S., Kong, M., Stetson, B. (2009).

Geographic socioeconomic status, health literacy, and perceived barriers to selfcare in at-risk, underserved adults with type 2 diabetes. Annals of Behavioral Medicine, 37 (Supplement), S197.

Meyer, J. A., Bonner, J., Rothschild, C., Rogers, W., Foster, M., Richardson, K., Robertson, E., Reitz, P., \& Stetson, B. (2008). Acceptance and experiential avoidance: Associations with quality of life and self-care in adults with type 2 diabetes. Annals of Behavioral Medicine, 35, (Supplement 1), S46.

Bonner, J., Stetson, B., Meyer, J., Rothschild, C., Ulmer, C., \& Beacham, A. (2006). The role of personality and schema in predicting exercise-related guilt. Annals of Behavioral Medicine, 31, (Supplement), S156.

Stetson, B., Beacham, A., Rothschild, C., Meyer, J., Bonner, J., \& Ulmer, C. (2005). Combined utility of the transtheoretical / stage of change and relapse prevention models in understanding the process of ongoing exercise. Annals of Behavioral Medicine, 29, (Supplement), S159.

Stetson, B., Beacham, A., Meyer, J., Ulmer, C., Rothschild, C., \& Bonner, J. (2005). Exercise cognitions differ by number of exercise relapse occurrences. Annals of Behavioral Medicine, 29 (Supplement), S160. 
Stetson, B., Ritchie, C., Adams, K, Marshall, K, \& Bonner, J. (2004). Pain, dependence, and psychosocial factors related to functional fitness in urban dwelling older women. Annals of Behavioral Medicine, 27 (Supplement), S049.

\section{UNDER REVIEW}

Rogers, W., Stetson, B., Bonner, J., Rothschild, C., Meyer, J., Krishnasamy, S., Richardson, K., and Mokshagundam, S. P. (Under review). Social isolation and neighborhood characteristics in low-income adults with type 2 diabetes: Relation to health indicators and quality of life.

\section{CONFERENCE PRESENTATIONS}

Meyer, J. A., Stetson, B. A., Bonner, J. E., Rothschild, C. L., \& Ulmer, C. S. (2006). Demoralization and affective state and associations with exercise characteristics in a sample of community exercisers. Poster presented at the $40^{\text {th }}$ Annual Meeting of the Association for Behavioral and Cognitive Therapies, November, 2006, Chicago, IL.

Stetson, B. A., O’Malley, K., Rothschild, C., Kostiwa, I., Rogers, J., \& Bonner, J. (2006). Environmental and affective associations with physical function in veterans receiving interdisciplinary home-based primary care. Poster presented at the $40^{\text {th }}$ Annual Meeting of the Association for Behavioral and Cognitive Therapies, November, 2006, Chicago, IL.

Bonner, J. E., Stetson, B., Beacham, A., Ulmer, C., Rothschild, C., \& Meyer, J. (2005). Associations between exercise schema and exercise behavior related to psychological well-being in community dwelling adults. Poster presented at the $39^{\text {th }}$ Annual Meeting of the Association for Behavioral and Cognitive Therapies, March, 2005, Washington, D.C.

Bonner, J. E., Ulmer, C. S., Buckley, A. F., Mitchell, C. K., Newton, T. L., \& WoodruffBorden, J. (2005). Differences in perceived health status among depressed, subclinically depressed, and non-depressed chronically-ill patients within a medically-underserved primary care population." Poster presented at the $39^{\text {th }}$ Annual Meeting of the Association for Behavioral and Cognitive Therapies, November, 2005, Washington, D.C.

Stetson, B., Bonner, J., Meyer, J., Ulmer, C., Rothschild, C., Kurian, R., \& Mokshagundam, S. P. (2005). Home-based physical activity behaviors in older men with diabetic neuropathy following cessation of supervised resistance training. Poster presented at the $39^{\text {th }}$ Annual Meeting of the Association for Behavioral and Cognitive Therapies, November, 2005, Washington, D.C. 
Weissbecker, I, Bonner, J. E., Beacham, A. O., Newton, T. L., Mitchell, C. K., \& Woodruff-Borden, J. (2004). Application of a manualized treatment approach for smoking cessation. Poster presented at the $38^{\text {th }}$ Annual Meeting of the Association for Advancement of Behavior Therapy, November, 2004, New Orleans, Louisiana.

Stetson, B., Schlundt, D., Mokshagundam, S. P, Bonner, J., \& Rothschild, C. (2004). Age differences in distress and perceived exercise barriers in mid-life and older adults with diabetes. Poster presented at the $38^{\text {th }}$ Annual Meeting of the Association for Advancement of Behavior Therapy, November, 2004, New Orleans, Louisiana.

Stetson, B., Beacham, A., Meyer, J., Bonner, J., Ulmer, C., \& Rothschild, C. (2004). Consistency of physical activity patterns and relationship to mood in community dwelling adults. Poster presented at the $38^{\text {th }}$ Annual Meeting of the Association for Advancement of Behavior Therapy, November 2004, New Orleans, Louisiana.

Markowski, V., Tarr, A., \& Bonner, J. (2002). Assessment of erectile function following perinatal exposure to an environmental anti-androgen. Poster presented at the $1^{\text {st }}$ Annual Scholarship and Creativity Fair, April, 2002, Portland, Maine.

\section{COMMUNITY PRESENTATIONS}

Bonner, J. (2009, March). Diabetes support group presentation: managing stress and type 2 diabetes. Clark Memorial Hospital, Jeffersonville, Indiana.

Bonner, J. (2007, April). Seminar on stress management and relaxation training with emphasis on mindfulness-based stress reduction. University of Louisville Hospital, Louisville, Kentucky.

\section{RESEARCH EXPERIENCE}

2008-2010

Doctoral Dissertation (defended 9/1/2010):

Dissertation Title: Social-cognitive predictors of physical activity initiation in type 2 diabetes following diabetes self-management education: Application of the Health Action Process Approach. Dissertation Chair: Barbara Stetson, Ph.D.

Committee: Benjamin Mast, Ph.D., Sri Prakash Mokshagundam, M.D., Tamara Newton, Ph.D., Paul Salmon, Ph.D.

2003-2009

Pre-doctoral Research Assistant, Health Behavior Change Lab, Department of Psychological and Brain Sciences, University of Louisville. 
Diabetes Self-Management Study. Study purpose: examination of psychological attitudes/beliefs towards diabetes self-care among type II diabetes outpatients undergoing Health Department Diabetes Self-Management Education classes. Responsibilities included study preparation of data collection materials, participant recruitment, data collection, participant reimbursement, and assist review of data entry, and assist data cleaning and analysis.

Exercise Maintenance following Completion of Structured Physical Activity Intervention in Veterans with Diabetes. Study purpose: examine unsupervised physical activity patterns among older-adult veterans with diabetes after completion of a structured, supervised physical activity intervention. Responsibilities included assisting the development of a theoretically-based intervention manual for physical activity for older male veterans with type II diabetes, assist in development of study materials, study recruitment $\&$ initial screening, processing of weekly data collection materials, follow-up phone-based data collection, assist in data entry, cleaning, and analysis.

Examination of the Relapse Prevention Model in Community Exercisers. Study purpose: examination of the Relapse Prevention Model applied to community exercisers (community-based sites and university undergraduate pool). Responsibilities included preparation of study materials, on-site recruitment and data collection across multiple sites, assist in data entry, cleaning, and analysis.

2002-2003

Research Assistant, WESTAT Inc., Portland, Maine. New England Study of Environment and Health (NESEH). Study purpose: NIH funded epidemiological study: investigating the prevalence of bladder cancer in New England. Responsibilities included study recruitment and in-field data collection throughout the state of Maine.

2001-2002

Undergraduate Research Assistant, Toxicology Lab, Department of Psychology, University of Southern Maine.

Effects of Pre-natal Anti-androgen exposure in animal models. Study purpose: examine effects of environmental anti-androgens on fetal development in animal models. Responsibilities included data collection using multiple modalities to assess sex-specific characteristics of Long-Evans hooded rats prenatally exposed to anti-androgens. 


\section{TEACHING EXPERIENCE}

\section{Instructor/Lecturer}

2008-2009 Instructor for undergraduate course: Abnormal Psychology, Department of Psychological and Brain Sciences, University of Louisville. Course overview: historical and contemporary understanding of assessment, diagnosis, and treatment of psychopathology and abnormal behavior. Responsible for development and teaching of course material, development, administration, and scoring of assignments/quizzes/exams, and recording/reporting of grades.

2005 Instructor for undergraduate/graduate level course: Introduction to Behavioral Medicine, Department of Psychological and Brain Sciences, University of Louisville. Course overview: comprehensive introduction to the theoretical and applied aspects of health psychology and behavioral medicine. Responsible for development and teaching of course material, development, administration, and scoring of assignments/quizzes/exams, and recording/reporting of grades.

1997-1999 Instructor - National Registry Emergency Medical Technician - Basic United States Air Force, Malcolm Grow Medical Center, Andrews AFB, Maryland. Assistant instructor responsible for teaching First Responder Awareness curriculum and assist in evaluating clinical practicum demonstrations for military and civilian fire and police personnel.

1994-1995 Assistant Instructor - Emergency Medical Technician 1-A, College of the Canyons, Valencia, California. Assisted in evaluating clinical practicum demonstrations. Class background: community-college undergraduate students.

\section{Teaching Assistant}

2007-2008 Teaching Assistant: undergraduate Drugs \& Behavior, Department of Psychological and Brain Sciences, University of Louisville.

Responsibilities included administration and grading of class assignments and exams, and occasional lecturing of course material.

2007-2008 Teaching Assistant: undergraduate Personality, Department of Psychological and Brain Sciences, University of Louisville. Responsibilities included administration and grading of class assignments and exams, and occasional lecturing of course material.

Teaching Assistant: undergraduate Personality, Department of Psychological and Brain Sciences, University of Louisville. 
Responsibilities included administration and grading of class assignments and exams, and occasional lecturing of course material.

2004-2005 Teaching Assistant: undergraduate Physiological Psychology, Department of Psychological and Brain Sciences, University of Louisville.

Responsibilities included administration and grading of class assignments and exams, and occasional lecturing of course material.

2003-2004 Teaching Assistant/Lab Instructor for undergraduate course: Introduction to Psychology, Department of Psychological and Brain Sciences, University of Louisville. Course overview: lab component of undergraduate course to reinforce concepts taught in course lecture. Responsible for class demonstrations and didactic instruction on specific introductory topics.

\section{HONORS AND AWARDS}

2003-2006

2002

1999

1999

1999

1998

1996
Annual Grawemeyer Research Award, University of Louisville Magna Cum Laude, University of Southern Maine

Honorable Separation, US Air Force

US Air Force Achievement Medal

US Air Force Good Conduct Medal

US Air Force Outstanding Unit Award

US Air Force Honor Graduate: Student Leadership School, Advanced Military Training 